\title{
Asset Prices with Heterogeneity in Preferences and Beliefs*
}

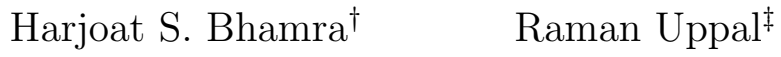

First version: March 2009

Last version: November 2010

This version: February 2013

${ }^{*}$ We are grateful for detailed comments from Michael Brennan, Bernard Dumas, Francisco Gomes, and Burton Hollifield. We would also like to acknowledge suggestions from Karim Abadir, Suleyman Basak, Pierluigi Balduzzi, Oliver Berndt, Andrea Buraschi, Georgy Chabakauri, David Chapman, Joao Cocco, Jakša Cvitanić, James Dow, Lorenzo Garlappi, Alan Kraus, Igor Makarov, Semyon Malamud, Anna Pavlova, Greg Vilkov, Hongjun Yan, and Yingzi Zhu, and from seminar participants at Boston College, École Polytechnique Fédérale de Lausanne, European Summer Symposium on Financial Markets at Gerzensee, Imperial College, Jackson Hole Finance Group, London Business School, London School of Economics, University of Cambridge, University of Oxford, University of Mannheim, Tel Aviv University, University of Pennsylvania, University of St. Gallen, the 2009 UBC Summer Finance Conference, the 2010 meetings of the American Finance Association, the European Finance Association, and the Jackson Hole Finance Group.

†Sauder School of Business, University of British Columbia, 2053 Main Mall, Vancouver BC, Canada V6T 1Z2; Email: harjoat.bhamra@sauder.ubc.ca.

${ }^{\ddagger}$ CEPR and Edhec Business School, 10 Fleet Place, Ludgate, London, United Kingdom EC4M 7RB; Email: raman.uppal@edhec.edu. 


\title{
Asset Prices with Heterogeneity in Preferences and Beliefs
}

First version: March 2009

Last version: November 2010

This version: January 2012

\begin{abstract}
In this paper, we study asset prices in a dynamic, continuous-time, general-equilibrium endowment economy where agents have "catching up with the Joneses" utility functions and differ with respect to their beliefs (because of differences in priors) and their preference parameters for time discount, risk aversion, and sensitivity to habit. A key contribution of our paper is to demonstrate how one can obtain a closed-form solution to the consumption-sharing rule for agents who have both heterogeneous priors and heterogeneous preferences without restricting the risk aversion of the two agents to special values. We solve in closed form also for the the state price density, the riskless interest rate and market price of risk; the stock price, equity risk premium, and volatility of stock returns; the term structure of interest rates; and the conditions necessary to obtain a stationary equilibrium in which both agents survive in the long run. The methodology we develop is sufficiently general that, as long as markets are complete, it can be applied to models set in discrete or continuous time, to endowment processes that are in the exponential affine jump-diffusion class, and, to settings with arbitrary updating of beliefs.
\end{abstract}




\section{Contents}

1 Introduction and Motivation 1

2 The Model 6

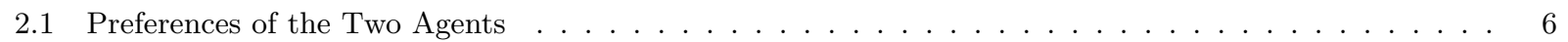

2.2 The Optimization Problem of Each Agent . . . . . . . . . . . . . . . . . . . . . 8

2.3 The Equilibrium . . . . . . . . . . . . . . . . . . . . . . . . . 9

3 Consumption Share, State Price Density and Asset Prices 9

3.1 The Equilibrium Consumption-Sharing Rule . . . . . . . . . . . . . . . . . . . . . . . 10

3.2 Equilibrium State Price Density . . . . . . . . . . . . . . . . . . . . . . . . . 12

3.3 Price-Dividend Ratio for Dividend Strips and Equity . . . . . . . . . . . . . . . . . . . . . . . . . . 14

4 Dynamics of the Consumption Share and State Price Density 17

4.1 The Processes for Aggregate Endowment and Beliefs of the Two Agents . . . . . . . . . . . . . . . 17

4.2 Definitions of Aggregate Preference Parameters and Beliefs . . . . . . . . . . . . . . . . . . . 18

4.3 Dynamics of the Consumption-Sharing Rule . . . . . . . . . . . . . . . . . . . . . . . . 20

4.4 Dynamics of State-Price Density: Risk-Free Rate and Market Price of Risk . . . . . . . . . . . . . . . 21

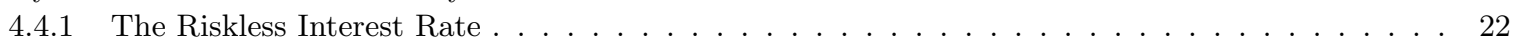

4.4 The Market Price of Risk . . . . . . . . . . . . . . . . . . . . . . . . . . . . . 24

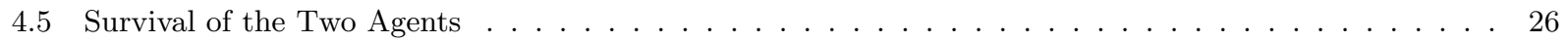

5 Asset Prices $\quad 27$

5.1 Valuation of Equity . . . . . . . . . . . . . . . . . . . . . . 27

5.2 Term Structure of Zero-Coupon Risky and Riskfree Claims . . . . . . . . . . . . . . . . . . . . 29

5.3 The Equity Risk Premium and Volatility of Stock-Market Returns . . . . . . . . . . . . . . . . . . 31

6 General Affine Model 32

7 Conclusion $\quad 34$

A Appendix: Lagrange's Theorem and Proofs for Propositions and Corollaries 36

A.1 Proof of Proposition 1: Consumption-sharing rule . . . . . . . . . . . . . . . . . . . 37

A.2 Proof of Proposition 2: State-price density . . . . . . . . . . . . . . . . . . . . . 40

A.3 Proof of Corollary 1: State-price density under identical risk aversion . . . . . . . . . . . . . . . . . 42

A.4 Proof of Proposition 3: Price of dividend strip . . . . . . . . . . . . . . . . . . . . . . . . 43

A.5 Proof of Corollary 2: Price of dividend strip with identical risk aversion . . . . . . . . . . . . . . 44

A.6 Proof of Proposition 4: Dynamics of the consumption-sharing rule . . . . . . . . . . . . . . 45

A.7 Proof of Proposition 5: Riskfree rate . . . . . . . . . . . . . . . . . . . . . 48

A.8 Proof of Corollary 3: Riskfree rate with correct beliefs or with identical risk aversions and habits . . . 50

A.9 Proof of Proposition 6: Market price of risk . . . . . . . . . . . . . . . . . . 50

A.10 Proof of Corollary 4: Market price of risk with correct beliefs or with identical risk aversions . . . . 51

A.11 Proof of Proposition $7 \ldots \ldots \ldots \ldots$

A.12 Proof of Proposition $8 \ldots \ldots \ldots \ldots \ldots$

A.13 Proof of Proposition 9: Long-term yield . . . . . . . . . . . . . . . . . . . . 62

A.14 Proof of Corollary 5: Survival and price impact under identical preferences and different beliefs. . . . . 65

A.15 Proof of Proposition 10: Risk premium and volatility of risky assets . . . . . . . . . . . . . . 65

A.16 Proof of Proposition $11 \ldots \ldots \ldots \ldots$

A.17 The Distribution of The Consumption Share . . . . . . . . . . . . . . . . . . . 70

References $\quad 73$ 


\section{List of corollaries, lemmas, propositions, and theorems}

Proposition $1 \quad$ Equilibrium share of consumption . . . . . . . . . . . . 11

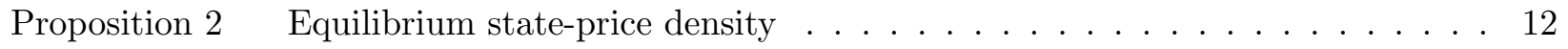

Corollary 1 Equilibrium state price density with identical risk aversion . . . . . . . . . 13

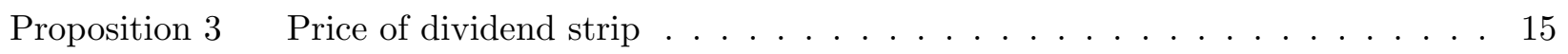

Corollary 2 Price of dividend strip with identical risk aversion . . . . . . . . . . . 16

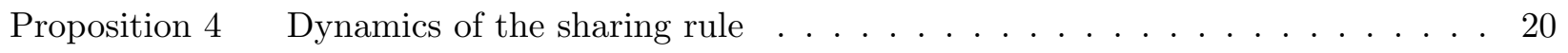

Proposition $5 \quad$ Locally riskfree interest rate . . . . . . . . . . . . . . 22

Corollary $3 \quad$ Locally riskfree interest rate - special cases . . . . . . . . . . . . . . . 22

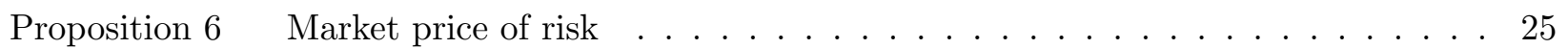

Corollary 4 Market price of risk - special cases . . . . . . . . . . . . . 25

Proposition $7 \quad$ Condition for almost-sure stationarity $\ldots \ldots \ldots \ldots \ldots$

Proposition $8 \quad$ Value of spanning assets $\phi_{n, 1, t, u}^{Y}$ and $\phi_{n, 2, t, u}^{Y} \ldots \ldots \ldots \ldots$

Proposition $9 \quad$ Long-term yield on risky and riskfree zero-coupon claims . . . . . . . . . . 29

Corollary $5 \quad$ Long-term yield when one agent has correct beliefs . . . . . . . . . . . 30

Proposition 10 Volatility of stock market returns and equity risk premium . . . . . . . . 31

Proposition 11 Values of $\phi_{n, 1, t, u}^{X}$ and $\phi_{n, 2, t, u}^{X}$ for general affine processes $\ldots \ldots . \ldots 33$

Theorem A1 Complex Analytic . . . . . . . . . . . . . . . 36

Theorem A2 Lagrange . . . . . . . . . . . . . . . . . . . 36

Lemma A1 Partial differential equation for price of a dividend strip . . . . . . . . . . 52

Lemma A2 Values of $\phi_{n, 1, t}^{X}$ and $\phi_{n, 2, t}^{X} \ldots \ldots \ldots \ldots \ldots \ldots \ldots$

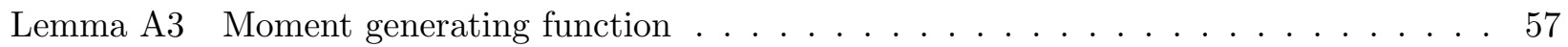

Proposition A1 Density function for the consumption share . . . . . . . . . . . 70 


\section{Introduction and Motivation}

Two key characteristics of economic agents are their beliefs and preferences. Our objective in this paper is to study the effect of heterogeneity in both of these characteristics on the choices of individual agents and the resulting asset prices. The agents we study have different beliefs about the growth rate of the aggregate endowment process and "catching up with the Joneses" utility functions with different parameters for time discount, risk aversion, and sensitivity to the historical standard of living. We show how to solve in closed form for optimal policies and asset prices of the stock and bond in a general equilibrium stochastic dynamic exchange economy with heterogeneous agents. ${ }^{1}$ This allows us to identify the strengths and limitations of the model with heterogeneity in both preferences and beliefs.

The importance of studying models with heterogeneous agents rather than a representative agent has been recognized by both policymakers and academics. For instance, the April 15, 2010 issue of the Economist describing the Soros-sponsored conference on "The Economic Crisis and the Crisis in Economics" says that, "The conference rehearsed many familiar complaints, bashing ... the use of representative agents (a kind of economic Everyman, whose behavior mimics the macroeconomy in microcosm)." Hansen (2010) in his talk at this conference lists one of the challenges for macroeconomic models to be "Building in explicit heterogeneity in beliefs, preferences ...." Stiglitz (2010) in his presentation at the same conference also criticizes the representative agent model and highlights the importance of heterogeneous agents as a key modeling challenge. Sargent (2008), in his presidential address to the American Economic Association, discusses extensively the implications of the common beliefs assumption for policy, and Hansen (2007, p. 27) in his Ely lecture says: "While introducing heterogeneity among investors will complicate model solution, it has intriguing possibilities. ... There is much more to be done." Empirical work by Beber, Buraschi, and Breedon (2009), Berrada and Hugonnier (2011), Buraschi and Jiltsov (2006), Buraschi, Trojani, and Vedolin (2009, 2010), and Ziegler (2007) also suggests the importance of allowing for heterogeneous beliefs and preferences in models of asset pricing.

\footnotetext{
${ }^{1}$ In particular, we obtain the following quantities in closed form: the equilibrium consumption allocation across agents and its dynamics over time; the state price density and its dynamics, which are characterized in terms of the riskless interest rate and the market price of risk; the stock price, the equity risk premium, and the volatility of stock returns; and, the term structure of interest rates and the term premium.
} 
A key contribution of our paper is to demonstrate how one can obtain a closed-form solution to the consumption-sharing rule for agents who have both heterogeneous priors and heterogeneous preferences without restricting the risk aversion of the two agents to special values. ${ }^{2}$ In the case of two agents, the consumption-sharing rule is a non-linear algebraic equation, which reduces to a polynomial of degree $\eta$ if the ratio of the risk aversion of one agent to that of the other is a natural number. If $\eta$ equals two, three or four, then this polynomial equation can of course be solved in closed-form. We show how to construct a closed-form solution for all real values of $\eta$ using a theorem due to Lagrange, and we solve in closed form not just for the interest rate and market price of risk, but also for the stock price, equity premium, volatility of stock market returns, and the term structure of interest rates when agents have heterogeneous preferences and beliefs. Thus, the model we analyze nests the models that consider an exchange economy with agents who have expected utility with different degrees of risk aversion, such as Wang (1996), Bhamra and Uppal (2009), and Weinbaum (2012), models with "catching up with the Joneses" utility functions, as in Chan and Kogan (2002) and Xiouros and Zapatero (2010), and they nest also the results in models where agents have expected utility with heterogeneous beliefs, for instance, Basak (2005) and Yan (2008).

The methodology we develop is sufficiently general that, as long as markets are complete, it can be used to identify the sharing rule in asset-pricing models set in discrete or continuous time; for endowment processes that are in the exponential affine jump-diffusion class; and, to settings with an arbitrary rule for updating of beliefs, including Bayesian learning. We consider the "catching up with the Joneses" utility function that has external habit but in contrast to Chan and Kogan (2002) and Xiouros and Zapatero (2010), we allow the sensitivity to the historical standard of living to be agent-specific. Our specification nests isoelastic and logarithmic utility functions, and is straightforward to apply to other time-additive utility functions, such as exponential and quadratic utility. Given the ubiquity of nonlinear sharing rules in solutions to problems in economics, finance and decision theory (see Peluso and Trannoy (2007) for examples of such problems), the approach we develop can be applied also to other problems, which previously would have called for numerical methods.

\footnotetext{
${ }^{2}$ Our work can be viewed as complementary to that of Calin, Chen, Cosimano, and Himonas (2005), who provide an analytic representation (that is, a convergent power series) for the price-dividend function of one state variable in an economy with a single representative agent whose utility function displays habit formation, and to Garlappi and Skoulakis (2011), who show how to exploit Taylor series expansions to solve portfolio choice problems in partial equilibrium.
} 
The paper that is closest to our work is Cvitanić, Jouini, Malamud, and Napp (2012), which also studies asset prices in an economy where agents have expected utility and differ with respect to both beliefs and their preference parameters. Their paper provides bounds on asset prices and characterizes prices in the limit when only one agent survives. However, it does not provide closedform solutions for these quantities. In fact, Cvitanić and Malamud (2009b, p. 3) write that:

"when risk aversion is heterogeneous, SDF [stochastic discount factor] is the solution to highly non-linear equation (1) [in their paper], and no explicit solution is possible, except for some very special values of risk aversion; see, for example, Wang (1996)."

In contrast to Cvitanić, Jouini, Malamud, and Napp (2012), we provide a closed-form solution for the stochastic discount factor without restricting the risk aversion of the two agents to special values and also allowing for learning. In particular, we show how the stochastic discount factor can be expressed as a weighted average of stochastic discount factors from a set of underlying single-agent economies. ${ }^{3}$ Muraviev (2012) extends the analysis in Cvitanić, Jouini, Malamud, and Napp (2012) to the case with learning and where agents have "catching up with the Joneses" utility functions considered in Chan and Kogan (2002) but where the sensitivity to the historical standard of living is agent specific, while Borovička (2012) extends the analysis to the case of recursive preferences.

Most of the other papers in the existing literature with heterogeneous agents allow for either differences in beliefs or differences in preferences. We first discuss the literature that considers heterogeneity in beliefs and then the literature that considers differences in preferences. Essentially, there are two ways to generate heterogeneity in beliefs. In the first approach, agents receive different information. This is the classical approach, adopted in the early noisy-rational-expectations literature with asymmetric information. ${ }^{4}$ In this class of models, one group of (informed) agents receives private signals and then there is a second group of agents (noise-traders), which trades for exogenous reasons and thereby prevents the price from fully revealing the private information of the informed agents. The second approach for generating heterogeneity, which is the one we adopt, is to have agents who "agree to disagree" about some aspect of the underlying economy, and in this class of models it is assumed that agents do not learn from each other's behavior. Morris

\footnotetext{
${ }^{3}$ We should point out that, in contrast to our analysis, which is for the case of two agents, the limit analysis of Cvitanić, Jouini, Malamud, and Napp (2012) considers an economy with more than two agents and derives interesting implications for the term structure of interest rates.

${ }^{4}$ See, for instance, Grossman and Stiglitz (1980), Hellwig (1980), Wang (1993), and Shefrin and Statman (1994)).
} 
(1995) provides a good philosophical discussion of this modeling approach. ${ }^{5}$ Excellent reviews of this literature are provided in Basak (2005) and Jouini and Napp (2007).

We now discuss the literature on the effect of heterogeneous preferences on asset prices. The effect of different time-discount factors on the efficient allocation of consumption is studied in Gollier and Zeckhauser (2005). The effect of heterogeneity in risk aversion on asset prices is examined in several papers, most of which assume that investors have expected utility. For example, Dumas (1989) studies the riskfree rate and the risk premium in a production economy; Wang (1996) examines the term structure in an exchange economy; Basak and Cuoco (1998) and Kogan, Makarov, and Uppal (2007) analyze the effect of constraints on borrowing and short-sales on the equity risk premium in an exchange economy; Bhamra and Uppal (2009) and Tran (2009) examine the volatility of stock market returns; Benninga and Mayshar (2000) and Weinbaum (2009) study option prices; Longstaff and Wang (2009) investigate the relation between open interest in the bond market and stock market returns; Cvitanić and Malamud (2009a,b,c) consider equilibrium with multiple heterogeneous traders who maximize utility of only terminal wealth; and, Garleânu and Panageas (2008) study the effect of heterogeneous preferences in an overlapping-generations model that leads to a stationary equilibrium. In contrast to these papers that assume investors have expected utility, Chan and Kogan (2002) and Xiouros and Zapatero (2010) study asset prices in an economy where agents have "catching-up-with-the-Joneses" preferences, where habit formation ensures that the model is stationary. And, finally there are papers that work with Epstein and Zin (1989) recursive preferences that allow for a distinction between risk aversion and the elasticity of intertemporal substitution. For example, Guvenen (2009), studies asset pricing in a model with heterogeneity in elasticity of intertemporal substitution, Isaenko (2008) studies the term structure in a model where agents differ in both their risk aversion and elasticity of intertemporal substitution, and Gomes and Michaelides (2008) study portfolio decisions of households and asset prices in a model where agents are heterogeneous not just in terms of preferences but are also exposed to uninsurable income shocks in the presence of borrowing constraints.

\footnotetext{
${ }^{5}$ Examples of papers using such models of incomplete information include Basak (2000), Beber, Buraschi, and Breedon (2009), Berrada (2006), Borovička (2012), Buraschi and Jiltsov (2006), Buraschi, Trojani, and Vedolin (2009, 2010), Cecchetti, Lam, and Mark (2000), David (2008), David and Veronesi (2002), Duffie, Garleânu, and Pedersen (2002), Dumas, Kurshev, and Uppal (2009), Gallmeyer (2000), Gallmeyer and Hollifield (2008), Kogan, Ross, Wang, and Westerfield (2006), Scheinkman and Xiong (2003), Veronesi (1999), Xiong and Yan (2010), Yan (2008), and Zapatero (1998). Yan (2008) also studies a model where agents have both heterogeneous beliefs and preferences, but he solves for asset prices in terms of exogenous variables only for the case where both agents have the same risk aversion, which is a natural number (see his Proposition 3).
} 
When there are multiple agents who differ in their risk aversion, there is no paper in the literature that provides a complete characterization of equilibrium that is exact and entirely analytical. For example, for the case of expected utility, Wang (1996) provides closed form expressions for only particular parameter values; Kogan and Uppal (2001) characterize the equilibrium in production and exchange economies approximately using perturbation analysis in the neighborhood of log utility; Bhamra and Uppal (2009) and Tran (2009) study stock-market-return volatility, but solve numerically for volatility; Dumas (1989) solves numerically for the interest rate in a production economy; for the case of "catching-up-with-the-Joneses" preferences, Chan and Kogan (2002) rely on numerical solutions, and the working-paper version of Chan and Kogan (2002) provides approximate analytic results in the neighborhood of log utility using perturbation analysis. Xiouros and Zapatero (2010) provide an expression for the value function of the central planner assuming a Gamma distribution for the risk tolerances of the investors, but asset prices are obtained using numerical methods. The models in Guvenen (2009), Isaenko (2008), and Gomes and Michaelides (2008) are also solved using numerical methods.

The rest of the paper is arranged as follows. In Section 2, we describe our model of an exchange economy with heterogeneous agents. The equilibrium consumption-sharing rule, derived by solving the problem of a "central planner," is given in Section 3 along with the state price density. Section 4 gives the dynamics of the state price density, which are defined in terms of the risk-free rate and the market price of risk. Asset prices, including the stock price, the volatility of stock market returns, and the equity and term premium are given in Section 5. In Section 6, we show how to price financial assets when the logarithm of aggregate endowment and agents' beliefs follow general affine processes, instead of the geometric Brownian motion assumed for the endowment process and the exponential martingale process assumed for beliefs in the previous section. We conclude in Section 7. Our main results are highlighted in propositions, results for special cases are given in corollaries, ${ }^{6}$ and detailed proofs for all the results, including a statement of Lagrange's Theorem, are provided in Appendix A.

\footnotetext{
${ }^{6}$ There are three special cases that we consider: one, where investors have identical beliefs but different preferences (risk aversion, rate of time preference, and sensitivity to habit); two, where investors differ in beliefs but have identical risk aversion; and three, where the investors differ in beliefs but have identical risk aversion and this risk aversion is a natural number.
} 


\section{The Model}

In this section, we describe the features of the economy we are considering. We consider a continuous-time, pure-exchange, Arrow-Debreu (complete markets) economy with an infinite time horizon. There is a single non-storable consumption good that serves as the numeraire and is modeled as an exogenously specified stochastic endowment process, $Y_{t}$ that is defined on a filtered probability space $\left(\Omega, \mathcal{F},\left\{\mathcal{F}_{t}\right\}, \mathbb{P}\right)$.

There are a large number of investors in the economy. These investors are of two types, which we denote by $k \in\{1,2\}$. We adopt the convention of subscripting by $k$ the quantities related to agents of type $k$, where $k \in\{1,2\}$. In order to be concise, instead of referring to these investors as "agents of type 1 and 2," we simply call them Agent 1 and Agent 2.

The utility function of Agent $k$ is denoted by $U_{k}$ and the beliefs of Agent $k$ are denoted by the probability measure $\mathbb{P}^{k}$. Our model differs from the standard representative-agent Lucas (1978) model along two dimensions: first, preferences of the two agents are heterogeneous; second, the two agents may not have the correct beliefs about the aggregate endowment process, and the beliefs of one agent may differ from those of the other. The sharing rule of the investor and the equilibrium state price density do not depend on the particular processes chosen for the dynamics of endowments and beliefs. For instance, the endowment process could be a geometric brownian motion that is typically assumed, but it could also be a much more complicated process that is not necessarily continuous or even affine. Similarly, while it is possible to assume that beliefs are not updated at all, one could also assume Bayesian updating or some form of non-Bayesian updating. The only assumption we need for the results regarding static quantities such as the sharing rule and the state price density is that financial markets are dynamically complete. Therefore, we specify the processes for endowments and beliefs only in Section 4, where we study the dynamics of the consumption share, state price density, and asset returns.

\subsection{Preferences of the Two Agents}

The consumption of Agent $k$ at instant $u$ is denoted by $C_{k, u}$ and the instantaneous utility from consumption is given by the following power function that depends on consumption relative to 
habit, $H_{k, u}$ :

$$
U_{k}\left(C_{k, u}, H_{k, u}\right) \equiv e^{-\beta_{k} u} \frac{1}{1-\gamma_{k}}\left(\frac{C_{k, u}}{H_{k, u}}\right)^{1-\gamma_{k}}
$$

where $\beta_{k}$ is the constant subjective discount rate (that is, the rate of time preference), and $\gamma_{k}$ is the degree of relative risk aversion. ${ }^{7}$ Without loss of generality, we assume that Agent 1's relative risk aversion is less than that of Agent 2: $\gamma_{1}<\gamma_{2}$.

The quantity $H_{k, u}$ in (1) can be interpreted as Agent $k$ 's sensitivity to the historical standard of living (external habit), as modeled in Muraviev (2012), which generalizes the specification in Chan and Kogan (2002) to allow for agent-specific habit. Under the approach in Muraviev,

$$
H_{k, u}=X_{u}^{h_{k}}=e^{h_{k} x_{u}}, \text { for } h_{k}>0,
$$

and $x_{u} \equiv \log X_{u}$. One can then define $x_{t}$ as the weighted geometric average of past realizations of the logarithm of the aggregate endowment process:

$$
x_{t}=x_{0} e^{-\lambda_{x} t}+\lambda_{x} \int_{0}^{t} e^{-\lambda_{x}(t-u)} y_{u} d u
$$

where $y_{u} \equiv \log Y_{u}$, and $Y_{u}$ denotes the aggregate endowment at time $u$. So, while $X_{u}$ represents the general index for the standard of living, the scalar $h_{k}$ determines the sensitivity of Agent $k$ to this index; if $h_{k}=1$ for all $k$, this reduces to the specification in Chan and Kogan (2002), and if $h_{k}=0$ one gets the standard isoelastic utility function without habit.

We define the distance between the logarithm of the aggregate endowment and its weighted geometric average as

$$
\omega_{t} \equiv y_{t}-x_{t}
$$

Observe that (2) implies that $d x_{t}=\lambda_{x}\left(y_{t}-x_{t}\right) d t$, and so the evolution of $\omega$ is given by

$$
d \omega_{t}=\lambda_{x}\left(\lambda_{x}^{-1} E_{t}\left[\frac{d y_{t}}{d t}\right]-\omega_{t}\right) d t+d y_{t}-E_{t}\left[d y_{t}\right]
$$

which makes it clear that $\omega$ exhibits mean reversion for any specification of the aggregate endowment.

\footnotetext{
${ }^{7}$ See Chan and Kogan (2002) for a discussion of this specification for the utility function, and why it is still appropriate to interpret $\gamma$ as the coefficient of relative risk aversion. For other papers in the literature that study the effect of habit on asset prices in representative-agent models, see Abel (1990, 1999) and Constantinides (1990).
} 
Chan and Kogan (2002) explain that $\gamma_{k}$ represents the relative risk aversion of Agent $k$. We explain below that if we parameterize $h_{k}$ as in (5) below

$$
h_{k} \equiv \frac{\gamma_{k}-\frac{1}{\psi_{k}}}{\gamma_{k}-1}
$$

then we can interpret $\frac{1}{\psi_{k}}$ as the sensitivity of the risk-free rate to the growth rate of aggregate consumption in the steady state when Agent $k$ is the sole agent in the economy without risk. ${ }^{8}$

\subsection{The Optimization Problem of Each Agent}

Given her beliefs, represented by the probability measure $\mathbb{P}^{k}$, the expected lifetime utility of Agent $k$ at time $t$ from consuming $C_{k, u}$ is given by

$$
V_{k, t}=E_{t}^{k}\left[\int_{t}^{\infty} e^{-\beta_{k}(u-t)} \frac{1}{1-\gamma_{k}}\left(\frac{C_{k, u}}{H_{k, u}}\right)^{1-\gamma_{k}} d u\right],
$$

where $E_{t}^{k}$ denotes the time- $t$ conditional expectation operator with respect to the probability measure $\mathbb{P}^{k}$.

The problem of Agent $k$ is to maximize lifetime utility, given by $V_{k, 0}$ in (6), subject to a static budget constraint, which restricts the present value of all future consumption to be no more than the initial wealth of each agent, denoted by $W_{k, 0}:^{9}$

$$
E_{0}^{k}\left[\int_{0}^{\infty} \frac{\pi_{k, t}}{\pi_{k, 0}} C_{k, t} d t\right] \leq W_{k, 0}
$$

${ }^{8}$ We start by considering the marginal utility (MU) of consumption at date $t$ for Agent $k$, which is given by

$$
\mathrm{MU}_{k, t}=e^{-\beta_{k} t} H_{k, t}^{\gamma_{k}-1} C_{k, t}^{-\gamma_{k}}
$$

When Agent $k$ is the sole agent in the economy, $C_{k}=Y$, and so her marginal utility can be written as:

$$
\mathrm{MU}_{k, t}=e^{-\beta_{k} t} e^{-\left(\gamma_{k}-\frac{1}{\psi_{k}}\right) \omega_{t}} e^{-\frac{1}{\psi_{k}} y_{t}}
$$

Thus, the instantaneous interest rate in the deterministic version of the economy is $r_{t}=-\ln M U_{t}=\beta_{k}+$ $\left(\gamma_{k}-\frac{1}{\psi_{k}}\right) \frac{d \omega_{t}}{d t}+\frac{1}{\psi_{k}} \frac{d y_{t}}{d t}$. From (4), we can see that in the deterministic version of the economy, $\omega$ possesses a steady state. At the steady state, $\frac{d \omega_{t}}{d t}=0$, so

$$
\frac{\left.\partial r_{t}\right|_{\frac{d \omega_{t}}{d t}=0}}{\partial\left(\frac{d y_{t}}{d t}\right)}=\frac{1}{\psi_{k}} .
$$

One might be tempted to think of $\psi_{k}$ as the elasticity of intertemporal substitution, but this interpretation would be accurate only in a model with internal habit.

${ }^{9}$ The budget constraint for Agent $k$ in (7) is written in terms of the state prices perceived by this agent; one could write an equivalent expression in terms of the state prices (and expectation) of the central planner. 
in which $\pi_{k, t}$ is the marginal utility of investor $k$ at date $u$ under the probability measure $\mathbb{P}^{k}$ :

$$
\pi_{k, t} \equiv \frac{\partial U\left(C_{k, t}\right)}{\partial C_{k, t}}=e^{-\beta_{k} t}\left(\frac{1}{H_{k, t}}\right)^{1-\gamma_{k}} C_{k, t}^{-\gamma_{k}}=e^{-\beta_{k} t} e^{\left(\gamma_{k}-\frac{1}{\psi_{k}}\right)\left(y_{t}-\omega_{t}\right)-\gamma_{k} \ln C_{k, t}} .
$$

\subsection{The Equilibrium}

The notion of equilibrium that we use is an extension of equilibrium in the single-agent model of Lucas (1978): both agents optimize their expected lifetime utility and all markets clear. Given our assumption that preferences are time separable and financial markets are complete, the dynamic consumption-portfolio choice problem simplifies to a static problem that requires one to choose the optimal allocation of consumption between the two investors for each date and state. If agents have identical beliefs, then one can solve for the equilibrium consumption policies by maximizing the social-welfare function of a "central-planner," which is a weighted average of the utility functions of individual agents, subject to the resource constraint that aggregate consumption is equal to aggregate endowment (dividends). In contrast to the case of identical beliefs, if agents have heterogeneous beliefs, Basak (2005) shows that the weights used to construct the central planner's utility function are stochastic. The central planner's utility function in this case is given by

$$
\sup _{C_{1}+C_{2} \leq Y} \sum_{k=1}^{2} \lambda_{k, t} U_{k}\left(C_{k, t}\right)
$$

where

$$
\lambda_{k, t}=\lambda_{k, 0} \xi_{k, t}
$$

and $\xi_{k, t}$ is the Radon-Nikodym derivative $d \mathbb{P}^{k} / d \mathbb{P}$, which relates Agent $k$ 's subjective beliefs to the true physical probability measure. ${ }^{10}$

\section{Consumption Share, State Price Density and Asset Prices}

Our main contribution is to derive a closed-form, convergent, series solutions for the sharing rule in Proposition 1 below, and to show in Proposition 2 how to construct the state-price density in the heterogeneous-agent economy in two simple steps. In the first step, one needs to obtain only

\footnotetext{
${ }^{10}$ If the state space for aggregate dividends were discrete, then $\xi_{k, t}$ would be the ratio of the probability that Agent $k$ assigns to a particular state, relative to the true probability of that state.
} 
the state price density for an economy where there is just one type of agent; this is obtained by computing the marginal utility of consumption for a single agent. In the second step, one needs to combine the state-price densities from the two single-agent economies to obtain the state price density in the heterogeneous-agent economy; we provide the formula for doing this. These results are derived without making specific assumptions about the process for aggregate endowment and how the beliefs of the two agents are updated.

Once we have obtained the state-price density for the heterogeneous-agent economy, the price for any asset can be obtained by integrating the product of the asset's payoff and the state-price density and taking expectations of that integral; we state this result in Proposition 3, and we demonstrate it for particular processes for endowments and beliefs in Section 5 and for general affine processes in Section 6.

\subsection{The Equilibrium Consumption-Sharing Rule}

The consumption-sharing rule, which shows how aggregate consumption is allocated between the two agents in equilibrium, is given by the first-order condition for optimal consumption for the central planner's problem in $(9)$ :

$$
\left(\lambda_{1,0} \xi_{1, t}\right) e^{-\beta_{1} t}\left(\frac{1}{H_{1, t}}\right)^{1-\gamma_{1}} C_{1, t}^{-\gamma_{1}}=\left(\lambda_{2,0} \xi_{2, t}\right) e^{-\beta_{2} t}\left(\frac{1}{H_{2, t}}\right)^{1-\gamma_{2}} C_{2, t}^{-\gamma_{2}} .
$$

In order to solve explicitly for the equilibrium allocations, we write Agent $k$ 's consumption

share as $\nu_{k, t} \equiv \frac{C_{k, t}}{Y_{t}}$, where $0 \leq \nu_{k} \leq 1$, and $\nu_{1}+\nu_{2}=1$. Then Equation (10) can be written as:

$$
\lambda_{1,0} \xi_{1, t} e^{-\beta_{1} t}\left(\frac{1}{H_{1, t}}\right)^{1-\gamma_{1}} Y_{t}^{-\gamma_{1}} \nu_{1, t}^{-\gamma_{1}}=\lambda_{2,0} \xi_{2, t} e^{-\beta_{2} t}\left(\frac{1}{H_{1, t}}\right)^{1-\gamma_{1}} Y_{t}^{-\gamma_{2}} \nu_{2, t}^{-\gamma_{2}}
$$

which can be rewritten as

$$
\pi_{t}=\hat{\pi}_{1, t} \nu_{1, t}^{-\gamma_{1}}=\hat{\pi}_{2, t} \nu_{2, t}^{-\gamma_{2}}
$$

where $\pi$ is the equilibrium state-price density, and

$$
\hat{\pi}_{k, t}=\lambda_{k, 0} \xi_{k, t} e^{-\beta_{k} t}\left(\frac{1}{H_{k, t}}\right)^{1-\gamma_{k}} Y_{t}^{-\gamma_{k}}=\lambda_{k, 0} \xi_{k, t} e^{-\beta_{k} t} e^{-\left(\gamma_{k}-\frac{1}{\psi_{k}}\right) \omega_{t}-\frac{1}{\psi_{k}} y_{t}}
$$


is the state-price density under the physical probability measure $\mathbb{P}$ when Agent $k$ is the sole agent in the economy. Thus, the consumption-sharing rule in (11) can be expressed as

$$
\begin{aligned}
\nu_{2, t}^{\eta} A_{t} & =\nu_{1, t}, \\
\text { where } \quad A_{t} & =\left(\frac{\hat{\pi}_{1, t}}{\hat{\pi}_{2, t}}\right)^{\frac{1}{\gamma_{1}}}, \\
\text { and } \quad \eta & =\gamma_{2} / \gamma_{1} .
\end{aligned}
$$

When $\eta \in\{1,2,3,4\}$, the expression in (13) can be written as a polynomial of degree 4 or less, thus allowing us to solve for the equilibrium consumption allocation in terms of radicals, using standard results from polynomial theory, as pointed out in Wang (1996). Because polynomials of order 5 and above do not admit closed-form solutions in terms of radicals, it would be appear that going beyond the results in Wang (1996) by solving for the consumption-sharing rule in closed-form when $\eta$ is a natural number greater than or equal to 5 is not possible. However, when $\eta$ is a natural number greater than or equal to 5 , the consumption shares can be obtained in closed-form by using hypergeometric functions. ${ }^{11}$ We go further still by showing that when $\eta$ is any real number, it is possible to derive closed-form, convergent, series solutions for the sharing rule. The series solutions are derived using a theorem of Lagrange (see Appendix A), which to the best of our knowledge has not been used before in finance or economics. However, Lagrange's Theorem does not provide the radius of convergence for the series, which is essential if we want to use these series to study the behavior of the consumption shares. We show, in the proof of Proposition 1, how to identify the radius of convergence: depending on whether $\frac{\hat{\pi}_{1, t}}{\hat{\pi}_{2, t}}<R$ or $\frac{\hat{\pi}_{1, t}}{\hat{\pi}_{2, t}}>R$, we get a different convergent series solution for the sharing rule and the solutions corresponding to these two regions are given in (14).

Proposition 1 (Equilibrium share of consumption) Agent 2's equilibrium share of the aggregate endowment, $\nu_{2, t}=\frac{C_{2, t}}{Y_{t}}$, is given by

$$
\nu_{2, t}=\left\{\begin{array}{cc}
\sum_{n=1}^{\infty} \frac{(-)^{n+1}}{n}\left(\frac{\hat{\pi}_{2, t}}{\hat{\pi}_{1, t}}\right)^{\frac{n}{\gamma_{2}}}\left(\begin{array}{c}
n \frac{\gamma_{1}}{\gamma_{2}} \\
n-1
\end{array}\right) \quad, \quad \frac{\hat{\pi}_{1, t}}{\hat{\pi}_{2, t}}>R \\
1-\sum_{n=1}^{\infty} \frac{(-)^{n+1}}{n}\left(\frac{\hat{\pi}_{1, t}}{\hat{\pi}_{2, t}}\right)^{\frac{n}{\gamma_{1}}}\left(\begin{array}{c}
n \frac{\gamma_{2}}{\gamma_{1}} \\
n-1
\end{array}\right), & , \frac{\hat{\pi}_{1, t}}{\hat{\pi}_{2, t}}<R
\end{array}\right.
$$

\footnotetext{
${ }^{11}$ See Abadir (1999) for an introduction to hypergeometric functions. Because the derivation of the sharing rule for the general case where $\eta$ is any real number is given in the appendix, the derivation showing how the sharing rule can be expressed in terms of hypergeometric functions when $\eta$ is a natural number greater than or equal to 5 is not included.
} 
where

$$
R=\frac{\gamma_{1}^{\gamma_{2}}}{\gamma_{2}^{\gamma_{2}}}\left(\frac{\gamma_{2}}{\gamma_{1}}-1\right)^{\gamma_{2}-\gamma_{1}}=\left(\frac{(\eta-1)^{\eta-1}}{\eta^{\eta}}\right)^{\gamma_{1}}
$$

and, for $z \in \mathbb{C}$ and $k \in \mathbb{N},\left(\begin{array}{l}z \\ k\end{array}\right)=\Pi_{j=1}^{k} \frac{z-k+j}{j}$ is the generalized binomial coefficient.

From the implicit expression for $\nu_{2, t}$ in (13) or the explicit expression in (14), we see also that the consumption shares of the two agents will depend on the ratio of the state-price densities in the single-agent economies:

$$
\frac{\hat{\pi}_{1, t}}{\hat{\pi}_{2, t}}=\frac{\lambda_{1,0}}{\lambda_{2,0}} e^{\left(\beta_{2}-\beta_{1}\right) t} \frac{\xi_{1, t}}{\xi_{2, t}} \frac{H_{2, t}^{1-\gamma_{2}}}{H_{1, t}^{1-\gamma_{1}}} Y_{t}^{\gamma_{2}-\gamma_{1}},
$$

which from (15) depends on the differences in initial endowments $\lambda_{k, 0}$, subjective discount rates $\beta_{k}$, beliefs $\xi_{k, t}$, sensitivities to the historical standard of living $H_{k, t}$, and risk aversions $\gamma_{k}$.

\subsection{Equilibrium State Price Density}

We now give the level of the equilibrium state-price density using convergent series. Equation (16) in the proposition below shows that the equilibrium state-price density can be expressed as a linear combination of state-price densities of single-agent economies, that is, $\hat{\pi}_{k, t}, k \in\{1,2\}$, defined in (12), where the individual terms $\hat{\pi}_{k, t}$ depend solely on exogenous variables.

Proposition 2 (Equilibrium state-price density) The equilibrium state-price density is given by

$$
\pi_{t}=\left\{\begin{array}{cl}
\sum_{n=0}^{\infty} a_{n, 1}^{\pi} \hat{\pi}_{1, t}^{1-\frac{n}{\gamma_{2}}} \hat{\pi}_{2, t}^{\frac{n}{\gamma_{2}}} & , \frac{\hat{\pi}_{1, t}}{\hat{\pi}_{2, t}}>R, \\
\sum_{n=0}^{\infty} a_{n, 2}^{\pi} \hat{\pi}_{1, t}^{\frac{n}{\gamma_{1}}}, \hat{\pi}_{2, t}^{1-\frac{n}{\gamma_{1}}} & , \frac{\hat{\pi}_{1, t}}{\hat{\pi}_{2, t}}<R,
\end{array}\right.
$$

where $a_{n, 1}^{\pi}=a_{n, 2}^{\pi}=1$ for $n=0$, and

$$
\begin{aligned}
& a_{n, 1}^{\pi}=\gamma_{1} \frac{(-1)^{n+1}}{n}\left(\begin{array}{c}
n \frac{\gamma_{1}}{\gamma_{2}}-\gamma_{1}-1 \\
n-1
\end{array}\right), n \in \mathbb{N}, \\
& a_{n, 2}^{\pi}=\gamma_{2} \frac{(-1)^{n+1}}{n}\left(\begin{array}{c}
n \frac{\gamma_{2}}{\gamma_{1}}-\gamma_{2}-1 \\
n-1
\end{array}\right), n \in \mathbb{N} .
\end{aligned}
$$

To understand the above proposition, note that $\hat{\pi}_{1, t}$ and $\hat{\pi}_{2, t}$ are the state-price densities for the

two single-agent economies. Then, $\hat{\pi}_{1, t}^{1-\frac{n}{\gamma_{2}}} \hat{\pi}_{2, t}^{\frac{n}{\gamma_{2}}}$ is the state-price density of an underlying economy, 
constructed as the Hölder mean of the state price densities from two single-agent economies. ${ }^{12}$ Finally, the equilibrium state-price density in (16) is a linear combination of the state-price densities of the underlying economies.

The expression for the equilibrium state-density in (16) can be simplified if agents have the same risk aversion, $\gamma_{1}=\gamma_{2}=\gamma$, and a further simplification is possible if $\gamma$ is a natural number. These simpler expressions are given in the corollary below.

Corollary 1 (Equilibrium state price density with identical risk aversion) Suppose agents have identical risk aversion, that is, $\gamma_{1}=\gamma_{2}=\gamma$, but different beliefs. Then the equilibrium stateprice density is given by

$$
\pi_{t}= \begin{cases}\sum_{n=0}^{\infty} a_{n}^{\pi} \hat{\pi}_{1, t}^{1-\frac{n}{\gamma}} \hat{\pi}_{2, t}^{\frac{n}{\gamma}}, & \hat{\pi}_{2, t}<\hat{\pi}_{1, t}, \\ \sum_{n=0}^{\infty} a_{n}^{\pi} \hat{\pi}_{1, t}^{\frac{n}{\gamma}} \hat{\pi}_{2, t}^{1-\frac{n}{\gamma}}, & \hat{\pi}_{2, t}>\hat{\pi}_{1, t},\end{cases}
$$

where, denoting by $\mathbb{N}_{0}$ the set of natural numbers that includes 0 ,

$$
a_{n}^{\pi}=\left(\begin{array}{c}
\gamma \\
n
\end{array}\right), n \in \mathbb{N}_{0}
$$

If relative risk aversion, $\gamma$, is a natural number, then the equilibrium state-price density can be further simplified to a finite sum:

$$
\begin{aligned}
\pi_{t} & =\sum_{n=0}^{\gamma} a_{n}^{\pi} \hat{\pi}_{1, t}^{1-\frac{n}{\gamma}} \hat{\pi}_{2, t}^{\frac{n}{\gamma}} \\
& =\left(\hat{\pi}_{1, t}^{\frac{1}{\gamma}}+\hat{\pi}_{2, t}^{\frac{1}{\gamma}}\right)^{\gamma} .
\end{aligned}
$$

Thus, the expression for the equilibrium state-price density in (22) is a power mean (with exponent $\frac{1}{\gamma}$ ) of the individual agent state-price densities. It follows from well known properties of the power mean that the state-price density in Equation (22) is increasing in relative risk aversion, $\gamma$. The intuition for this is that more risk averse agents will be more willing to pay for a unit of consumption in a given state. If $\gamma=1$, the power mean reduces to the arithmetic mean; if $\gamma \rightarrow \infty$ it reduces to the geometric mean; and, if $\gamma \rightarrow 0$, it reduces to the maximum of the individual-agent state-price densities.

\footnotetext{
${ }^{12}$ If $p$ is a non-zero real number, the Hölder mean of $a$ and $b$ with weights $w$ and $1-w$, and exponent $p$ is: $\left(w a^{p}+(1-w) b^{p}\right)^{\frac{1}{p}}$, and when $p \rightarrow 0$, the Hölder mean reduces to $a^{w} b^{1-w}$.
} 
The special case considered in Corollary 1 where $\gamma_{1}=\gamma_{2}=\gamma$, with $\gamma$ being a natural number, is similar to the model studied in Dumas, Kurshev, and Uppal (2009, Equation (35)), where they obtain a similar expression for the state price density. Because $\gamma$ needs to be a natural number, this special case does not allow one to study the case of risk aversion smaller than one. Our Proposition 2, in contrast, allows for different risk aversion parameters for the two agents and does not restrict their values to be natural numbers.

\subsection{Price-Dividend Ratio for Dividend Strips and Equity}

In this section, we first identify the price of a dividend strip, which is a claim that pays a single cashflow of $Y_{u}$ at a particular time $u .{ }^{13}$ We denote the date- $t$ price of this dividend strip by $V_{t, u}^{Y}$, where

$$
V_{t, u}^{Y}=Y_{t} v_{t, u}^{Y}
$$

where the price-dividend ratio of the dividend strip is

$$
v_{t, u}^{Y}=E_{t}\left[\frac{\pi_{u}}{\pi_{t}} \frac{Y_{u}}{Y_{t}}\right]
$$

We can then obtain the price of equity by integrating the price of dividend strips over time. To see this, let $P_{t}^{Y}$ denote the price of the single share of the risky asset (stock), which is a claim on the aggregate dividend, $Y_{t}$. The stock price is given by

$$
P_{t}^{Y}=Y_{t} p_{t}^{Y}
$$

where the price-dividend ratio for equity $p_{t}^{Y}$ is:

$$
p_{t}^{Y}=E_{t} \int_{t}^{\infty} \frac{\pi_{u}}{\pi_{t}} \frac{Y_{u}}{Y_{t}} d u=\int_{t}^{\infty} v_{t, u}^{Y} d u
$$

We derive a representation for the price-dividend ratio of the dividend strip, $v_{t, u}^{Y}$, by using the state-price density in Proposition 2. Because the state-price density is one of two linear combinations of state-price densities from a set of underlying economies, depending on whether $\frac{\hat{\pi}_{1, u}}{\hat{\pi}_{2, u}} \gtrless R$, the price-dividend ratio of the dividend strip, $v_{t, u}^{Y}$, is a sum of two weighted averages. The first

\footnotetext{
${ }^{13}$ In the appendix, we show how to value more general cashflow payments.
} 
is a weighted average of price-dividend ratios from a set of underlying economies conditional on $\frac{\hat{\pi}_{1, u}}{\hat{\pi}_{2, u}}>R$, and the second is a weighted average of price-dividend ratios from a set of underlying economies conditional on $\frac{\hat{\pi}_{1, u}}{\hat{\pi}_{2, u}}<R$.

Proposition 3 (Price of dividend strip) The time-t price of the dividend strip, which pays the cash flow $Y_{u}$ at date $u>t$, is given by $V_{t, u}^{Y}=v_{t, u}^{Y} Y_{t}$, where

$$
v_{t, u}^{Y}=\sum_{n=0}^{\infty} \omega_{n, 1, t} \phi_{n, 1, t, u}^{Y}+\sum_{n=0}^{\infty} \omega_{n, 2, t} \phi_{n, 2, t, u}^{Y}
$$

where the weights $\omega_{n, 1, t}, n \in \mathbb{N}_{0}$, and $\omega_{n, 2, t}, n \in \mathbb{N}_{0}$, are given by

$$
\begin{aligned}
& \omega_{n, 1, t}=a_{n, 1}^{\pi}\left(\nu_{1, t}^{\gamma_{1}}\right)^{1-\frac{n}{\gamma_{2}}}\left(\nu_{2, t}^{\gamma_{2}}\right)^{\frac{n}{\gamma_{2}}}, n \in \mathbb{N}_{0} \\
& \omega_{n, 2, t}=a_{n, 2}^{\pi}\left(\nu_{1, t}^{\gamma_{1}}\right)^{\frac{n}{\gamma_{1}}}\left(\nu_{2, t}^{\gamma_{2}}\right)^{1-\frac{n}{\gamma_{1}}}, n \in \mathbb{N}_{0},
\end{aligned}
$$

and each set of weights sums to one:

$$
\sum_{n=0}^{\infty} \omega_{n, 1, t}=\sum_{n=0}^{\infty} \omega_{n, 2, t}=1
$$

and $\phi_{n, 1, t, u}^{Y}\left(\phi_{n, 2, t, u}^{Y}\right)$ is the price-dividend ratio for the "spanning asset" which pays the cashflow $Y_{u}$ at date $u$, provided $\frac{\hat{\pi}_{1, u}}{\hat{\pi}_{2, u}}>R\left(\frac{\hat{\pi}_{1, u}}{\hat{\pi}_{2, u}}<R\right)$,

$$
\begin{aligned}
& \phi_{n, 1, t, u}^{Y}=E_{t}\left[\frac{\hat{\pi}_{1, u}^{1-\frac{n}{\gamma_{2}}} \hat{\pi}_{2, u}^{\frac{n}{\gamma_{2}}}}{1-\frac{n}{\gamma_{2}} \hat{\pi}_{2, t}^{\frac{n}{\gamma_{2}}}} \frac{Y_{u}}{Y_{t}} 1_{\left\{\frac{\hat{\pi}_{1, u}}{\hat{\pi}_{2, u}}>R\right\}}\right], n \in \mathbb{N}_{0}, \\
& \phi_{n, 2, t, u}^{Y}=E_{t}\left[\frac{\hat{\pi}_{1, u}^{\frac{n}{\gamma_{1}}} \hat{\pi}_{2, u}^{1-\frac{n}{\gamma_{1}}}}{\hat{\pi}_{1, t}^{\frac{n}{\gamma_{1}}} \hat{\pi}_{2, t}^{1-\frac{n}{\gamma_{1}}}} \frac{Y_{u}}{Y_{t}} 1_{\left\{\frac{\hat{\pi}_{1, u}}{\hat{\pi}_{2, u}}<R\right\}}\right], n \in \mathbb{N}_{0} .
\end{aligned}
$$

The above proposition is useful because we see from (25) that we have reduced the problem of finding the value of dividend strips for the heterogeneous-agent economy to the problem of finding the value of the assets $\phi_{n, 1, t, u}^{Y}$ and $\phi_{n, 2, t, u}^{Y}$ in the underlying economies, with state-price densities $\hat{\pi}_{1, t}^{1-\frac{n}{\gamma_{2}}} \hat{\pi}_{2, t}^{\frac{n}{\gamma_{2}}}$ and $\hat{\pi}_{1, t}^{\frac{n}{\gamma_{1}}} \hat{\pi}_{2, t}^{1-\frac{n}{\gamma_{1}}}$, respectively. In other words, from (25) we can see that the value of a dividend strip is spanned by the asset values, $\phi_{n, 1, t, u}^{Y}$ and $\phi_{n, 2, t, u}^{Y}$, leading us to call them "spanning 
assets". Furthermore, by virtue of (24), we can see that the same spanning assets can be used to value equity; that is the price-dividend ratio for equity is:

$$
p_{t}^{Y}=\sum_{n=0}^{\infty} \omega_{n, 1, t} \int_{t}^{\infty} \phi_{n, 1, t, u}^{Y} d u+\sum_{n=0}^{\infty} \omega_{n, 2, t} \int_{t}^{\infty} \phi_{n, 2, t, u}^{Y} d u
$$

The power of this result rests on the fact that computing price-dividend ratios directly using (23) or (24) involves solving a valuation problem with an endogenous state variable $\nu_{1, t}$, whereas computing price-dividend ratios indirectly via Proposition 3 eliminates the endogenous state variable because the values of the spanning assets are determined in the underlying economies where the state-price density is the Hölder mean of the state-price density of homogeneous-agent economies.

We now consider two special cases: the first where the two agents have the same risk aversion, $\gamma_{1}=\gamma_{2}=\gamma$, and the second, where the two agents have the same risk aversion and $\gamma$ is a natural number.

Corollary 2 (Price of dividend strip with identical risk aversion) When risk aversions are identical, $\gamma_{1}=\gamma_{2}=\gamma$, then

$$
v_{t, u}^{Y}=\sum_{n=0}^{\infty} \omega_{n, 1, t} \phi_{n, 1, t, u}^{Y}+\sum_{n=0}^{\infty} \omega_{n, 2, t} \phi_{n, 2, t, u}^{Y}
$$

where

$$
\begin{aligned}
& \omega_{n, 1, t}=\left(\begin{array}{l}
\gamma \\
n
\end{array}\right)\left(\nu_{1, t}^{\gamma}\right)^{1-\frac{n}{\gamma}}\left(\nu_{2, t}^{\gamma}\right)^{\frac{n}{\gamma}}, n \in \mathbb{N}_{0} \\
& \omega_{n, 2, t}=\left(\begin{array}{l}
\gamma \\
n
\end{array}\right)\left(\nu_{1, t}^{\gamma}\right)^{\frac{n}{\gamma}}\left(\nu_{2, t}^{\gamma}\right)^{1-\frac{n}{\gamma}}, n \in \mathbb{N}_{0},
\end{aligned}
$$

and each set of weights sums to one: $\sum_{n=0}^{\infty} \omega_{n, 1, t}=\sum_{n=0}^{\infty} \omega_{n, 2, t}=1$.

If in addition to risk aversions being identical, $\gamma_{1}=\gamma_{2}=\gamma$, we also have that $\gamma \in \mathbb{N}$, then the above expressions simplify further to:

$$
v_{t}^{Y}=\sum_{n=0}^{\gamma} \omega_{n, t} v_{n, t, u}^{Y}
$$

where

$$
v_{n, t, u}^{Y}=E_{t}\left[\frac{\hat{\pi}_{1, u}^{1-\frac{n}{\gamma}} \hat{\pi}_{2, u}^{\frac{n}{\gamma}}}{\hat{\pi}_{1, t}^{1-\frac{n}{\gamma}} \hat{\pi}_{2, t}^{\frac{n}{\gamma}}} \frac{Y_{u}}{Y_{t}}\right], n \in \mathbb{N}_{0} \text { and } n \leq \gamma
$$




$$
\omega_{n, t}=\left(\begin{array}{c}
\gamma \\
n
\end{array}\right)\left(\nu_{1, t}^{1-\frac{n}{\gamma}} \nu_{2, t}^{\frac{n}{\gamma}}\right)^{\gamma}
$$

and $\sum_{0}^{\gamma} \omega_{n, t}=1$

From (33), we see that the price-dividend ratio of the dividend strip in the economy with heterogenous beliefs is a weighted sum of the price-dividend ratios of the dividend strips in $1+\gamma$

underlying economies, where in the $n$ 'th such economy, the state-price density, $\hat{\pi}_{1, t}^{1-\frac{n}{\gamma}} \hat{\pi}_{2, t}^{\frac{n}{\gamma}}$, is the Hölder mean of the state-price densities in the single-agent economies. The special case considered in Corollary 2 is similar to the model studied by Yan (2008, Proposition 3), where he obtains closed-form results for only the case in which the risk aversion parameter $\gamma$ is identical across agents and $\gamma$ is a natural number, which then excludes the case of risk aversion smaller than one. Our Proposition 3, in contrast, allows for different risk aversion parameters for the two agents and does not restrict their values to be natural numbers.

Below, in Proposition 8 of Section 5, we derive explicit expressions for $\phi_{n, 1, t, u}^{Y}$ and $\phi_{n, 2, t, u}^{Y}$ by making particular assumptions for the dynamics of the aggregate endowment process and beliefs. In Section 6, we show how to characterize $\phi_{n, 1, t, u}^{Y}$ and $\phi_{n, 2, t, u}^{Y}$ for affine processes.

\section{Dynamics of the Consumption Share and State Price Density}

So far in our analysis, we have not specified particular processes for aggregate dividends and beliefs. However, to characterize the dynamics of the consumption share, the state price density, and asset prices, we need to specify the dynamics for aggregate dividends and beliefs of the two agents, which we do in Section 4.1. Then, in Section 4.3 we derive the implications of heterogeneity for the dynamics of the sharing rule and the dynamics of the state price density.

\subsection{The Processes for Aggregate Endowment and Beliefs of the Two Agents}

The true evolution of the aggregate endowment, $Y$, is assumed to be:

$$
\frac{d Y_{t}}{Y_{t}}=\mu_{Y} d t+\sigma_{Y} d Z_{t}, \quad Y_{0}>0
$$

in which $\mu_{Y}$ and $\sigma_{Y}$ are constants and $Z$ is a one-dimensional Brownian motion. 
Agent $k$ believes that the expected growth rate of the endowment process takes the constant value, $\mu_{Y, k}$, as in Yan (2008), Borovička (2012), and Fedyk, Heyerdahl-Larsen, and Walden (2012). Therefore, defining the quantity $\sigma_{\xi, k} \equiv \frac{\mu_{Y, k}-\mu_{Y}}{\sigma_{Y}}$, Agent $k$ 's beliefs can be represented by an exponential martingale

$$
\xi_{k, t}=e^{-\frac{1}{2} \sigma_{\xi, k}^{2} t+\sigma_{\xi, k} Z_{t}},
$$

which can be written as: ${ }^{14}$

$$
\frac{d \xi_{k, t}}{\xi_{k, t}}=\sigma_{\xi, k} d Z_{t}
$$

Hence, by Girsanov's Theorem, Agent $k$ believes that the process for aggregate endowments is

$$
\frac{d Y_{t}}{Y_{t}}=\mu_{Y, k} d t+\sigma_{Y} d Z_{k, t}
$$

where $Z_{k, t}=Z_{t}-\sigma_{\xi, k} t$ is a standard Brownian motion under $\mathbb{P}^{k}$, which represents Agent $k$ 's beliefs. Hence, we see that the expected growth rate of the aggregate endowment under $\mathbb{P}^{k}$ is $\mu_{Y, k} .{ }^{15}$

We quantify the level of disagreement between the two agents via the process, $\xi_{t}$, where $\xi_{t} \equiv$ $\frac{\xi_{2, t}}{\xi_{1, t}}=e^{-\frac{1}{2}\left(\sigma_{\xi, 2}^{2}-\sigma_{\xi, 1}^{2}\right) t+\left(\sigma_{\xi, 2}-\sigma_{\xi, 1}\right) Z_{t}}$, and its dynamics are

$$
\frac{d \xi_{t}}{\xi_{t}}=\mu_{\xi} d t+\sigma_{\xi} d Z_{t}
$$

where

$$
\begin{aligned}
\mu_{\xi} & \equiv-\sigma_{\xi, 1}\left(\sigma_{\xi, 2}-\sigma_{\xi, 1}\right), \\
\sigma_{\xi} & \equiv\left(\sigma_{\xi, 2}-\sigma_{\xi, 1}\right)=\frac{\mu_{Y, 2}-\mu_{Y, 1}}{\sigma_{Y}} .
\end{aligned}
$$

\subsection{Definitions of Aggregate Preference Parameters and Beliefs}

In this section, we define the aggregate preferences in the economy and also aggregate beliefs, which will then be used in the expressions for the dynamics of the sharing rule and the dynamics of the state price density.

Definition 1 (Aggregate risk aversion) The aggregate relative risk aversion, $\mathbf{R}_{t}$, in the economy is defined as the consumption-share-weighted harmonic mean of individual agents' relative risk

\footnotetext{
${ }^{14}$ The exponential martingale, $\xi_{k, t}$, defines the probability measure $\mathbb{P}^{k}$ on $(\Omega, \mathcal{F})$, via $\mathbb{P}^{k}\left(e_{T}\right)=E_{t}\left[1_{e_{T}} \xi_{k, T}\right], \forall t, T \in$ $[0, \infty), t \leq T$, where $e_{T}$ is an event which occurs at time $T$ and $\mathbb{P}^{k}\left(e_{T}\right)$ is the probability of its occurrence based on information known at time $t$.

${ }^{15}$ Note that the probability measures $\mathbb{P}^{1}, \mathbb{P}^{2}$ and $\mathbb{P}$ are all equivalent; that is, they agree on which events are impossible.
} 
aversions:

$$
\mathbf{R}_{t}=\left(\nu_{1, t} \frac{1}{\gamma_{1}}+\nu_{2, t} \frac{1}{\gamma_{2}}\right)^{-1} .
$$

Equivalently, the aggregate risk tolerance in the economy, $1 / \mathbf{R}_{t}$, is the consumption-share-weighted arithmetic mean of individual agents' risk tolerances, $1 / \gamma_{k}$.

Defining $w_{k}$ to be the consumption-share weighted relative risk tolerances of investor $k$ :

$$
w_{k}=\frac{\frac{1}{\gamma_{k}} \nu_{k, t}}{\frac{1}{\gamma_{1}} \nu_{1, t}+\frac{1}{\gamma_{2}} \nu_{2, t}}, \text { and } w_{1}+w_{2}=1,
$$

we can then define the aggregate rate of time preference, aggregate prudence, and aggregate beliefs as follows.

Definition 2 (Aggregate rate of time preference) The aggregate rate of time preference in the economy, $\boldsymbol{\beta}_{t}$, is given by the weighted arithmetic mean of individual agents' rates of time preference, where the weights are the consumption-share weighted relative risk tolerances of the two investors given in Equation (39):

$$
\boldsymbol{\beta}_{t}=w_{1, t} \beta_{1}+w_{2, t} \beta_{2}
$$

Definition 3 (Aggregate prudence) The quantity $\mathbf{P}_{t}$ is the aggregate prudence in the economy: $:^{16}$

$$
\mathbf{P}_{t}=\left(1+\gamma_{1}\right)\left(\frac{\mathbf{R}_{t}}{\gamma_{1}}\right)^{2} \nu_{1, t}+\left(1+\gamma_{2}\right)\left(\frac{\mathbf{R}_{t}}{\gamma_{2}}\right)^{2} \nu_{2, t}
$$

Definition 4 (Aggregate belief) The aggregate belief, $\boldsymbol{\mu}_{Y, t}$, is given by the weighted arithmetic mean of the beliefs of individual agents,

$$
\boldsymbol{\mu}_{Y, t}=w_{1, t} \mu_{Y, 1}+w_{2, t} \mu_{Y, 2},
$$

where the weights are the consumption-share weighted relative risk tolerances of the two investors, as defined in Equation (39).

\footnotetext{
${ }^{16}$ Note that aggregate prudence may be larger than the prudence of either agent; that is, aggregate prudence is not necessarily bounded between the prudence of the individual agents. Consequently, the interest rate in the two-agent economy, which depends on aggregate prudence as shown in Equation (48), may not be bounded between the interest rates in the economies with only one of the two agents, as observed in Wang (1996).
} 


\subsection{Dynamics of the Consumption-Sharing Rule}

We are now ready to describe the evolution of the consumption-sharing rule.

Proposition 4 (Dynamics of the sharing rule) The true evolution of the sharing rule is given by:

$$
\frac{d \nu_{1, t}}{\nu_{1, t}}=\mu_{\nu_{1, t}} d t+\sigma_{\nu_{1, t}} d Z_{t}
$$

where

$$
\begin{aligned}
\sigma_{\nu_{1, t}} & =\frac{\nu_{2, t} \mathbf{R}_{t}}{\gamma_{1} \gamma_{2}}\left[\frac{\mu_{Y, 1}-\mu_{Y, 2}}{\sigma_{Y}}+\left(\gamma_{2}-\gamma_{1}\right) \sigma_{Y}\right] \\
\mu_{\nu_{1, t}} & =\nu_{2, t} \frac{\mathbf{R}_{t}}{\gamma_{1} \gamma_{2}}\left\{\beta_{2}-\beta_{1}+\left(\frac{\frac{\mu_{Y, 1}+\mu_{Y, 2}}{2}-\mu_{Y}}{\sigma_{Y}}\right)\left(\frac{\mu_{Y, 2}-\mu_{Y, 1}}{\sigma_{Y}}\right)\right. \\
& +\left(\frac{1}{\psi_{2}}-\frac{1}{\psi_{1}}\right)\left(\mu_{Y}-\frac{1}{2} \sigma_{Y}^{2}\right)+\left[\left(\gamma_{2}-\frac{1}{\psi_{2}}\right)-\left(\gamma_{1}-\frac{1}{\psi_{1}}\right)\right] \lambda_{x}\left(\bar{\omega}-\omega_{t}\right) \\
& \left.+\frac{1}{2} \frac{\gamma_{2} w_{2, t}^{2}-\gamma_{1} w_{1, t}^{2}}{\gamma_{1} \gamma_{2}}\left[\left(\frac{\mu_{Y, 1}-\mu_{Y, 2}}{\sigma_{Y}}\right)^{2}+2\left(\mu_{Y, 1}-\mu_{Y, 2}\right)\left(\gamma_{2}-\gamma_{1}\right)+\left(\gamma_{2}-\gamma_{1}\right)^{2} \sigma_{Y}^{2}\right]\right\}
\end{aligned}
$$

From (40), we see that the volatility of the sharing rule, $\sigma_{\nu_{1, t}}$, is driven by differences in risk aversion and differences in beliefs, but not differences in subjective discount rates or habit, which have only a deterministic effect and so appear only in the expression for the drift of the sharing rule, $\mu_{\nu_{1, t}}$. The expression for $\sigma_{\nu_{1, t}}$ in (40) shows that, if agents have identical beliefs, then an increase in heterogeneity in risk aversion leads to an increase in the volatility of the consumption share of Agent 1 because of an increase in consumption risk sharing. Similarly, if agents have identical risk aversions $\left(\gamma_{1}=\gamma_{2}\right)$, then an increase in heterogeneity in beliefs leads to an increase in the volatility of the consumption share of Agent $1 .{ }^{17}$

However, when both risk aversion and beliefs are heterogeneous, then the effect of an increase in the heterogeneity in either one of these factors on the volatility of the consumption share depends on whether it reinforces or counteracts the effect of the other factor. From (40) we observe that $\sigma_{\nu_{1, t}}>0$ if and only if

$$
\gamma_{2}-\gamma_{1}>\frac{\mu_{Y, 2}-\mu_{Y, 1}}{\sigma_{Y}^{2}}
$$

\footnotetext{
${ }^{17}$ In the case where agents have different risk aversion but the same beliefs, $\sigma_{\nu_{1, t}}$ is always positive.
} 
that is, if the more risk averse agent is not too optimistic relative to the less risk averse agent. If this condition is satisfied, then we see from the definition of aggregate risk aversion in (38) that $\mathbf{R}_{t}$ will be countercyclical, because when the aggregate endowment has a positive shock, the weight on the risk aversion of Agent 1 increases, and so the aggregate risk aversion in the economy decreases. Therefore, the heterogeneity in risk aversion and beliefs can generate countercyclical aggregate risk aversion endogenously. Moreover, if Agent 2, who has the higher risk aversion, is also the more pessimistic agent, then the heterogeneity in beliefs reinforces the effect arising from heterogeneity in risk aversion. This countercyclical behavior of aggregate risk aversion has previously been recognized in the multiagent models of Chan and Kogan (2002) and Xiouros and Zapatero (2010), where agents have heterogeneous risk aversions but homogeneous beliefs, and this feature appears in the single-agent model of Campbell and Cochrane (1999) as a consequence of the assumption of habit-formation.

Equation (41) shows how $\mu_{\nu_{1, t}}$ depends on differences in subjective discount rates and differences in beliefs. The impact of differences in $\psi_{k}$ is given in (42). We also see how $\mu_{\nu_{1, t}}$ is affected by the volatility of aggregate endowment growth, $\sigma_{Y}$, and the differences in beliefs, both of which appear in $(43) .{ }^{18}$

\subsection{Dynamics of State-Price Density: Risk-Free Rate and Market Price of Risk}

In this section, we determine the dynamics of the state-price density, and hence, the equilibrium riskfree rate and market price of risk.

The central planner's state-price density, $\pi_{t}$, is given by

$$
\pi_{t}=\hat{\pi}_{k, t} \nu_{k, t}^{-\gamma_{k}}=\xi_{k, t} \pi_{k, t}, \quad k \in\{1,2\}
$$

where $\hat{\pi}_{k}$ is the state-price density in a homogeneous-agent economy where all agents are of type $k$ and is defined in (12), while $\pi_{k}$ is the state-price density of Agent $k$ in the heterogeneous-agent economy, and is defined in (8). ${ }^{19}$

\footnotetext{
${ }^{18}$ The discussion above illustrates the benefit of having the closed-form results in Propositions 1 and 4 . Because we have explicit expressions for the sharing rule and its dynamics, we can understand exactly how these are affected by the parameters for preferences, beliefs, and the endowment process.

${ }^{19}$ Because financial markets are effectively complete, marginal utilities of consumption are equal across agents for each state, and therefore the first order condition for consumption in (10) ensures that the expression in (45) is the same for $k \in\{1,2\}$.
} 
From standard results in asset pricing (see Duffie (2001, Section 6.D, p. 106)), the evolution of the central planner's state-price density $\pi_{t}$ is:

$$
\frac{d \pi_{t}}{\pi_{t}}=-r_{t} d t-\theta_{t} d Z_{t}
$$

and the evolution of Agent $k$ 's state-price density, $\pi_{k, t}$, is:

$$
\frac{d \pi_{k, t}}{\pi_{k, t}}=-r_{t} d t-\theta_{k, t} d Z_{k, t}
$$

where, if $B_{t}$ denote the price of a locally risk-free bond in zero net supply, then, the riskfree return $r_{t}$ is given by $\frac{d B_{t}}{B_{t}}=r_{t} d t$.

Note from (47) that each agent has her own market price of risk, $\theta_{k}$; however, because the instantaneously riskfree bond is a traded security, the two agents must agree on its price, and hence, on the interest rate.

\subsubsection{The Riskless Interest Rate}

The following proposition gives the expression for the riskfree rate.

Proposition 5 (Locally riskfree interest rate) The locally riskfree rate is given by:

$$
\begin{aligned}
r_{t} & =\boldsymbol{\beta}_{t}+\mathbf{R}_{t} \sum_{k=1}^{2} w_{k, t} \mu_{Y, k}-\frac{1}{2} \mathbf{R}_{t} \mathbf{P}_{t} \sigma_{Y}^{2}-\left(\mathbf{R}_{t}-\sum_{k=1}^{2} \frac{1}{\psi_{k}} w_{k, t}\right) \lambda_{x} \omega_{t} \\
& +\frac{1}{2} w_{1, t} w_{2, t}\left(1-\frac{\mathbf{R}_{t}}{\gamma_{1} \gamma_{2}}\right) \sigma_{\xi}^{2}-w_{1, t} w_{2, t} \mathbf{R}_{t}\left(\frac{1}{\gamma_{1}}-\frac{1}{\gamma_{2}}\right)\left(\mu_{Y, 1}-\mu_{Y, 2}\right),
\end{aligned}
$$

where $\omega_{t}$ is defined in $(3), \bar{\omega}_{k}=\frac{\mu_{Y, k}-\frac{1}{2} \sigma_{Y}^{2}}{\lambda_{x}}$ is the long-run mean of $\omega_{t} \equiv y_{t}-x_{t}$ under the beliefs of Agent $k$, that is, under the probability measure $\mathbb{P}^{k}$, and the weights $w_{k}$ are defined in (39).

The corollary below gives the riskfree rate for some special cases.

Corollary 3 (Locally riskfree interest rate - special cases) If agents have identical and correct beliefs, then the locally riskfree rate is given by

$$
r_{t}=\boldsymbol{\beta}_{t}+\mathbf{R}_{t} \mu_{Y}-\frac{1}{2} \mathbf{R}_{t} \mathbf{P}_{t} \sigma_{Y}^{2}-\left(\mathbf{R}_{t}-\sum_{k=1}^{2} \frac{w_{k, t}}{\psi_{k}}\right) \lambda_{x} \omega_{t}
$$


On the other hand, if $\gamma_{1}=\gamma_{2}=\gamma$ and $\psi_{1}=\psi_{2}=\psi$, but agents have different beliefs and rates of time preference, then the locally riskfree rate is given by

$$
r_{t}=\sum_{k=1}^{2} \nu_{k, t} \beta_{k}+\gamma \sum_{k=1}^{2} \nu_{k, t} \mu_{Y, k}-\frac{1}{2} \gamma(1+\gamma) \sigma_{Y}^{2}-\left(\gamma-\frac{1}{\psi}\right) \lambda_{x} \omega_{t}+\frac{1}{2} \nu_{1, t} \nu_{2, t}\left(1-\frac{1}{\gamma}\right) \sigma_{\xi}^{2}
$$

To interpret the expression for the interest rate, recall that in a standard Lucas (1978) economy where all agents have correct and identical beliefs, and identical preferences that are given by a power function, the expression for the interest rate is

$$
\hat{r}_{k}=\beta_{k}+\gamma_{k} \mu_{Y}-\frac{1}{2} \gamma_{k}\left(1+\gamma_{k}\right) \sigma_{Y}^{2}
$$

From Equation (52), we see that the interest is positively related to the rate of impatience, $\beta_{k}$; positively related to the growth rate of aggregate endowment, $\mu_{Y}$, scaled by risk aversion $\gamma_{k}$; and the third term arises because of precautionary savings in the face of aggregate endowment risk, which leads to a drop in the interest rate, where the magnitude of the drop depends on $\left(1+\gamma_{k}\right)$, the relative prudence of agents, and on risk aversion, $\gamma_{k}$.

When investors are identical but their preferences exhibit habit and their beliefs $\mu_{Y, k}$ are allowed to deviate from the true growth rate $\mu_{Y}$, then the interest rate is given by

$$
\hat{r}_{k, t}=\beta_{k}+\gamma \mu_{Y, k}-\frac{1}{2} \gamma_{k}\left(1+\gamma_{k}\right) \sigma_{Y}^{2}-\left(\gamma_{k}-\frac{1}{\psi_{k}}\right) \lambda_{x} \omega_{t}
$$

Comparing (53) with (52) we see that the first term, $\beta_{k}$, is the same in both expressions; in the second term, the belief of each agent about the growth rate of aggregate endowments, $\mu_{Y, k}$, replaces the true growth rate, $\mu_{Y}$; the third term, which reflects the effect of the precautionary savings, is the same; and, the fourth term is new, and it reflects the effect of habit.

Equation (50) of the corollary shows that if only risk aversions are heterogeneous but beliefs are homogeneous and correct, then the riskfree rate has the same form as in (53) for a single-agent economy, but with the aggregate quantities $\boldsymbol{\beta}_{t}, \mathbf{R}_{t}$, and aggregate prudence, $\mathbf{P}_{t}$, replacing their single-agent counterparts; note, however, that because the weights used to construct these aggregate measures vary over time, the aggregate measures will be time-varying rather than constant.

On the other hand, if only beliefs are heterogeneous but preferences are homogeneous, then we see from the last term in (51) that if $\gamma<1$ the differences in beliefs will decrease the interest 
rate, or equivalently, increase the price of the instantaneously riskless bond. This effect is similar to the premium ("bubble") in asset prices that has been studied in Harrison and Kreps (1978) and Scheinkman and Xiong (2003) for the case of risk neutrality $(\gamma=0)$ in the presence of shortsale constraints; over here, we get a similar effect for agents who are risk averse without needing to constrain shortsales. However, if $\gamma>1$ then the price of the bond decreases with heterogeneity in beliefs, an observation made also in Dumas, Kurshev, and Uppal (2009).

When agents have both heterogeneous beliefs and preferences, the risk-free rate is given in lines (48) and (49). The first three terms on line (48) correspond to the three terms in (50), and are related to the subjective time preference of the two agents, the growth rate of aggregate endowment, and the demand for precautionary savings. The last term on line (48) arises because of habit. The two terms on line (49) arise because of differences in beliefs. The first term in (49) increases the risk-free rate when the aggregate risk aversion is less than the square of the geometric mean of risk aversion; that is, $\mathbf{R}_{t}<\gamma_{1} \gamma_{2}$, which is true if and only if $\gamma_{1}>1 .^{20}$ It follows that if $\gamma_{1}>1$ $\left(\gamma_{1}<1\right)$, then heterogeneity in beliefs increases (decreases) the risk-free rate. The second term

arises because of differences in both risk aversion and in beliefs; that is, $\left(\frac{1}{\gamma_{1}}-\frac{1}{\gamma_{2}}\right)\left(\mu_{Y, 1}-\mu_{Y, 2}\right)$. When the less risk averse agent is also the more optimistic agent, that is, $\mu_{Y, 1}>\mu_{Y, 2}$, this term decreases the risk-free rate.

One of the limitations of the representative-agent general-equilibrium model of asset pricing is that, when risk aversion is increased in order to improve the match of the equity risk premium in the model to that in the data, the riskfree interest rate in the model becomes too high relative to the data; this is the "riskfree rate puzzle" identified in Weil (1989). From the discussion above, we see that both heterogeneity in beliefs and preferences have the potential to reduce the interest rate relative to a homogeneous agent economy.

\subsubsection{The Market Price of Risk}

From (46), we see that the volatility of the central planner's state price density is given by the market price of risk, $\theta_{t}$, while from (47) we see that the volatility of the state price density for each individual agent is given by the perceived market price of risk, $\theta_{k, t}$. The following proposition gives the expressions for these market prices of risk.

\footnotetext{
${ }^{20}$ Note that since $\mathbf{R}_{t} \leq \gamma_{2}, \mathbf{R}_{t}<\gamma_{1} \gamma_{2}$ if and only if $\gamma_{1}>1$.
} 
Proposition 6 (Market price of risk) The market price of risk of the central planner, $\theta_{t}$, is:

$$
\theta_{t}=\mathbf{R}_{t} \sigma_{Y}+\frac{\mu_{Y}-\boldsymbol{\mu}_{Y, t}}{\sigma_{Y}}
$$

and the market price of risk perceived by Agent $k$ is:

$$
\theta_{k, t}=\mathbf{R}_{t} \sigma_{Y}+\frac{\mu_{Y, k}-\boldsymbol{\mu}_{Y, t}}{\sigma_{Y}}, k \in\{1,2\}
$$

The corollary below gives the market prices of risk for the central planner and the two agents for the special cases where agents have identical preferences or identical beliefs.

Corollary 4 (Market price of risk - special cases) If agents have identical and correct beliefs, then the central planner's market price of risk, $\theta_{t}$, and the market price of risk perceived by Agent $k, \theta_{k, t}$ are given by:

$$
\theta_{t}=\theta_{k, t}=\mathbf{R}_{t} \sigma_{Y}, k \in\{1,2\}
$$

On the other hand, if agents have identical relative risk aversion, $\gamma_{1}=\gamma_{2}=\gamma$, but different beliefs, rates of time preference, and $\psi_{k}$, then the central planner's equilibrium market price of risk is

$$
\theta_{t}=\gamma \sigma_{Y}+\frac{\mu_{Y}-\boldsymbol{\mu}_{Y, t}}{\sigma_{Y}}
$$

and the market price of risk perceived by Agents $k$ is

$$
\theta_{k, t}=\gamma \sigma_{Y}+\frac{\mu_{Y, k}-\boldsymbol{\mu}_{Y, t}}{\sigma_{Y}}
$$

To understand the expressions for the market price of risk in the above corollary and proposition, note that in an economy where all agents have correct and identical beliefs, and identical risk aversion, $\gamma_{1}=\gamma_{2}=\gamma$, the market price of risk is $\theta=\gamma \sigma_{Y}$. When only preferences are different across agents, then $\gamma$ is replaced by the average risk aversion in the economy, $\mathbf{R}_{t}$, and the market price of risk is given by (56), with both agents agreeing with this market price of risk. On the other hand, if preferences are identical but beliefs are heterogeneous, then we see that agents do not agree on the market price of risk. From (57) we see that if Agent 2 is pessimistic relative to Agent 1, $\mu_{Y, 1}>\mu_{Y, 2}$, then the market price of risk perceived by Agent 1 will be increased. The magnitude of this increase depends on the consumption-share of Agent $2, \nu_{2, t}$, because this 
determines Agent 2's influence on equilibrium stock market returns. For the general case in (55) where both beliefs and risk aversions are different, we see that the increase in the market price of risk perceived by Agent 1 will depend on the consumption share of Agent 2, $\nu_{2, t}$, and the agent's risk tolerance, $1 / \gamma_{2}$, relative to aggregate risk tolerance in the economy, $1 / \mathbf{R}_{t}$, because these are the two factors that determine the size of the position Agent 2 takes in the stock market. Finally, from the expression in (54) for the general case where there is heterogeneity in both preferences and beliefs, we see that the market price of risk for the central planner will increase if average beliefs are pessimistic; that is, $\mu_{Y}>\boldsymbol{\mu}_{Y, t}$. The intuition for this is that, if agents are pessimistic on average, then the compensation for bearing risk must be relatively higher than what it needs to be in an economy where agents have the correct average beliefs.

Note also that the market price of risk is countercyclical in the data and in the model of Campbell and Cochrane (1999). This will be true also in our model if $\mathbf{R}_{t}$ is countercyclical, which requires that the more risk averse agent not be too optimistic relative to the less risk averse agentthe exact condition is given in Equation (44).

\subsection{Survival of the Two Agents}

In this section, we derive the conditions under which both agents survive in the long run. We say that the economy is stationary if both agents survive. To formalize the concept of survival, we introduce the concept of almost-sure (a.s.) survival with respect to a particular probability measure, as in Kogan, Ross, Wang, and Westerfield (2006) and Yan (2008).

Definition 5 Agent $k$ survives $\mathbb{P}$-a.s. if

$$
\lim _{t \rightarrow \infty} \nu_{k, t}>0, \mathbb{P}-a . s
$$

Note that if an agent's consumption share is strictly above zero with a probability of less than one, under $\mathbb{P}$ say, then she does not survive $\mathbb{P}$-almost surely. Furthermore, the probability measure is important, because an agent may believe she survives almost surely (with respect to the probability measure representing her beliefs), when in fact, she almost surely does not survive under the true probability measure $\mathbb{P}^{21}$

\footnotetext{
${ }^{21}$ Agent $k$ survives $\mathbb{P}^{j}$-a.s. if $\lim _{t \rightarrow \infty} \nu_{k, t}>0, \mathbb{P}^{j}$-a.s.
} 
The following proposition provides a condition for almost sure survival under $\mathbb{P}$, extending the results in Yan (2008) along two dimensions: introducing preferences with external habit and allowing for heterogeneity in relative risk aversion and habit.

Definition 6 (Survival index) Agent $k$ has a survival index $I_{k}$, defined by

$$
I_{k}=\beta_{k}+\frac{1}{2}\left(\frac{\mu_{Y, k}-\mu_{Y}}{\sigma_{Y}}\right)^{2}+\frac{1}{\psi_{k}}\left(\mu_{Y}-\frac{1}{2} \sigma_{Y}^{2}\right), k \in\{1,2\}
$$

It is important to note that relative risk aversion does not affect the survival index in any way. This is because of the effect of habit, as explained in Chan and Kogan (2002).

Proposition 7 (Condition for almost-sure stationarity) The economy is almost surely stationary under $\mathbb{P}$ if and only if the following two conditions are satisfied:

$$
\begin{aligned}
\text { Condition 1: } & I_{1}=I_{2} ; \\
\text { Condition 2: } & \mu_{Y, 1}-\frac{1}{\psi_{1}} \sigma_{Y}^{2}=\mu_{Y, 2}-\frac{1}{\psi_{2}} \sigma_{Y}^{2} .
\end{aligned}
$$

\section{Asset Prices}

In this section, we derive the stock price, the equity risk premium, the volatility of stock market returns, and the term structure of interest rates for the particular aggregate endowment process and beliefs specified in Equations (35) and (36), and then examine how these quantities are influenced by heterogeneity in beliefs, rates of time preference, habit, and risk aversion. In Section 6, we extend these pricing results to the case where the logarithm of aggregate endowment and agents' beliefs follow general affine processes, as opposed to the particular processes assumed in Equations (35) and (36).

\subsection{Valuation of Equity}

Recall from Equation (25) in Proposition 3 that the date- $t$ price of a dividend strip which pays out $Y_{u}$ units of consumption at date $u>t$, denoted by $V_{t, u}^{Y}$, can be obtained from the prices of the spanning assets. For the aggregate endowment and beliefs, given in Equations (35) and (36), the value of the spanning assets $\phi_{n, k, t, u}^{Y}$ can be expressed analytically. Denote the cumulative standard normal distribution function by $\Phi(z)=\int_{-\infty}^{z} \frac{e^{-\frac{1}{2} x^{2}}}{\sqrt{2 \pi}} d x$. We then have the following result. 
Proposition 8 (Value of spanning assets $\phi_{n, 1, t, u}^{Y}$ and $\phi_{n, 2, t, u}^{Y}$ ) Given the aggregate endowment process specified in Equation (35) and the beliefs process in (36), the values of $\phi_{n, 1, t, u}^{Y}$ and $\phi_{n, 2, t, u}^{Y}$ that pay $Y_{u}$ at $u$ whenever $\frac{\hat{\pi}_{1, u}}{\hat{\pi}_{2, u}}>R$ and $\frac{\hat{\pi}_{1, u}}{\hat{\pi}_{2, u}}<R$, respectively, are:

$$
\begin{aligned}
& \left.\phi_{n, 1, t, T}^{Y}=v_{t, u}^{Y, h o m}\left(k_{1}, b_{1}\right) e^{a_{1}\left[\hat{\mu}_{q, 1}^{Y}(T-t)+\frac{1}{2} a_{1} \sigma_{q}^{2}(T-t)+b_{1} \frac{1-e^{-\lambda_{x}(T-t)}}{\lambda_{x}} \sigma_{q} \sigma_{Y}\right]}\right]_{\Phi}\left(\frac{B_{1}^{Y}\left(q_{t}, \omega_{t}, T-t\right)-\rho}{\sqrt{2 A_{1}\left(q_{t}, \omega_{t}, T-t\right)}}\right), \\
& \left.\phi_{n, 2, t, T}^{Y}=v_{t, u}^{Y, h o m}\left(k_{2}, b_{2}\right) e^{a_{2}\left[\hat{\mu}_{q, 2}^{Y}(T-t)+\frac{1}{2} a_{2} \sigma_{q}^{2}(T-t)+b_{2} \frac{1-e^{-\lambda_{x}(T-t)}}{\lambda_{x}} \sigma_{q} \sigma_{Y}\right.}\right]_{\Phi}\left(\frac{\rho-B_{2}^{Y}\left(q_{t}, \omega_{t}, T-t\right)}{\sqrt{2 A_{2}\left(q_{t}, \omega_{t}, T-t\right)}}\right),
\end{aligned}
$$

where

$$
\begin{aligned}
& v_{t, u}^{Y, h o m}\left(k_{j}, b_{j}\right)=e^{-k_{j}(T-t)+b_{j}\left(\omega_{t}-\hat{\omega}_{j}^{Y}\right)\left[1-e^{-\lambda_{x}(T-t)}\right]+\frac{1}{2} b_{j}^{2} \frac{1-e^{-2 \lambda_{x}(T-t)}}{2 \lambda_{x}} \sigma_{Y}^{2}}, \\
& \hat{k}_{j}=\beta_{j}+\frac{1}{\psi_{j}} \mu_{Y, j}-\frac{1}{2} \frac{1}{\psi_{j}}\left(1+\frac{1}{\psi_{j}}\right) \sigma_{Y}^{2}+\frac{1}{\psi_{j}} \sigma_{Y}^{2}-\mu_{Y, j}, \\
& a_{1}=-\frac{n}{\gamma_{2}}, \quad a_{2}=\frac{n}{\gamma_{1}} \\
& b_{1}=-\left[\frac{n}{\gamma_{2}}\left(\gamma_{2}-\frac{1}{\psi_{2}}\right)+\left(1-\frac{n}{\gamma_{2}}\right)\left(\gamma_{1}-\frac{1}{\psi_{1}}\right)\right] \text {, } \\
& b_{2}=-\left[\left(1-\frac{n}{\gamma_{2}}\right)\left(\gamma_{2}-\frac{1}{\psi_{2}}\right)+\frac{n}{\gamma_{1}}\left(\gamma_{1}-\frac{1}{\psi_{1}}\right)\right] \text {, } \\
& \rho=\ln R . \\
& A_{k}\left(q_{t}, \omega_{t}, T-t\right)=\frac{1}{2}\left[\sigma_{q}^{2}(T-t)+2 d_{\omega} \frac{1-e^{-\lambda_{x}(T-t)}}{\lambda_{x}} \sigma_{q} \sigma_{Y}+d_{\omega}^{2} \frac{1-e^{-2 \lambda_{x}(T-t)}}{2 \lambda_{x}} \sigma_{Y}^{2}\right], \\
& B_{k}^{Y}\left(q_{t}, \omega_{t}, T-t\right)=q_{t}+\hat{\mu}_{k, q}^{Y}(T-t)+d_{\omega}\left[e^{-\lambda_{x}(T-t)} \omega_{t}+\left(1-e^{-\lambda_{x}(T-t)}\right) \hat{\omega}_{k}^{Y}\right], \\
& +a_{k} \sigma_{q}^{2}(T-t)+\left(b_{k}+a_{k} d_{\omega}\right) \frac{1-e^{-\lambda_{x}(T-t)}}{\lambda_{x}} \sigma_{q} \sigma_{Y}+b_{k} d_{\omega} \frac{1-e^{-2 \lambda_{x}(T-t)}}{2 \lambda_{x}} \sigma_{Y}^{2}, \\
& \hat{\mu}_{k, q}^{Y}=\mu_{q}+\left(\left(1-\frac{1}{\psi_{k}}\right) \sigma_{Y}+\sigma_{\xi, k}\right) \sigma_{q} \\
& \hat{\omega}_{k}^{Y}=\frac{\mu_{Y, k}-\frac{1}{2} \sigma_{Y}^{2}+\left(1-\frac{1}{\psi_{k}}\right) \sigma_{Y}^{2}}{\lambda_{x}} .
\end{aligned}
$$

To understand the intuition behind the prices of the spanning assets, it is useful to define the yield of a spanning asset via:

$$
y_{n, j, T-t}^{\phi, Y}=-\frac{1}{T-t} \ln \phi_{n, j, t, T}^{Y}
$$


Hence,

$$
\begin{aligned}
y_{n, 1, T-t}^{\phi, Y} & =-\frac{1}{T-t} \ln v_{t, u}^{Y, h o m}\left(k_{1}, b_{1}\right)-a_{1}\left[\hat{\mu}_{q, 1}^{Y}+\frac{1}{2} a_{1} \sigma_{q}^{2}+b_{1} \frac{1-e^{-\lambda_{x}(T-t)}}{\lambda_{x}(T-t)} \sigma_{q} \sigma_{Y}\right] \\
& -\frac{1}{T-t} \ln \Phi\left(\frac{B_{1}^{Y}\left(q_{t}, \omega_{t}, T-t\right)-\rho}{\sqrt{2 A_{1}\left(q_{t}, \omega_{t}, T-t\right)}}\right) .
\end{aligned}
$$

First note that the yield in a homogeneous agent economy where all agents are of Type 1 would be

$$
-\frac{1}{T-t} \ln v_{t, u}^{Y, h o m}\left(k_{1},-\left(\gamma_{1}-\frac{1}{\psi_{1}}\right)\right)
$$

We can then see that the first term in $(63),-\frac{1}{T-t} \ln v_{t, u}^{Y, h o m}\left(k_{1}, b_{1}\right)$, is the expression for the yield in a homogeneous agent economy where all agents are of Type 1, with one change: the difference between the relative risk aversion and the inverse of the sensitivity of the risk-free rate to consumption growth in the homogeneous agent economy is replaced by its weighted average, that is, $-b_{1}$, where $b_{1}$ is defined in (62). Given that the spanning asset is priced using the Hölder mean of the state-price densities of single agent economies, with weights $\frac{n}{\gamma_{2}}$ and $1-\frac{n}{\gamma_{2}}$, it is natural that the same weights appear in (62). The second term in (63) adjusts for the impact of heterogeneity on the distribution of consumption. The final term stems from $\Phi\left(\frac{B_{1}^{Y}\left(q_{t}, \omega_{t}, T-t\right)-\rho}{\sqrt{2 A_{1}\left(q_{t}, \omega_{t}, T-t\right)}}\right)$, the risk-adjusted probability of the underlying cash flow for the spanning asset being paid. An analagous interpretation holds for $y_{n, 2, T-t}^{\phi, Y}$.

\subsection{Term Structure of Zero-Coupon Risky and Riskfree Claims}

We now explore the term structure of zero-coupon risky and riskfree claims in the presence of heterogeneity in beliefs and preferences. We start by defining the yield on a zero-coupon risky claim, $y_{T-t}^{Y}$ :

$$
y_{T-t}^{Y}=-\frac{1}{T-t} \ln \frac{V_{T-t}^{Y}}{Y_{t}} .
$$

The following proposition gives this yield when the maturity of the claim is infinite, that is, the "long-term" yield.

Proposition 9 (Long-term yield on risky and riskfree zero-coupon claims) The long-term yield on the risky zero-coupon claim, $y_{T-t}^{Y}$, which pays the random cash flow $Y_{T}$ at time $T$ is given 
by

$$
\lim _{T \rightarrow \infty} y_{T-t}^{Y}=\min _{k \in\{1,2\}}\left(\beta_{k}+\frac{1}{\psi_{k}} \mu_{Y, k}-\frac{1}{2} \frac{1}{\psi_{k}}\left(1+\frac{1}{\psi_{k}}\right) \sigma_{Y}^{2}+\frac{1}{\psi_{k}} \sigma_{Y}^{2}-\mu_{Y, k}\right) .
$$

The long-term yield on the riskfree zero-coupon discount bond, $y_{T-t}^{1}$, as $T \rightarrow \infty$ is

$$
\lim _{T \rightarrow \infty} y_{T-t}^{1}=\min _{k \in\{1,2\}}\left(\beta_{k}+\frac{1}{\psi_{k}} \mu_{Y, k}-\frac{1}{2} \frac{1}{\psi_{k}}\left(1+\frac{1}{\psi_{k}}\right) \sigma_{Y}^{2}\right)
$$

and the limit of the term premium, the difference between $y_{T-t}^{1}$ and the short rate, $r_{t}$, is:

$$
\lim _{T \rightarrow \infty} y_{T-t}^{1}-r_{t}=\min _{k \in\{1,2\}}\left(\beta_{k}+\frac{1}{\psi_{k}} \mu_{Y, k}-\frac{1}{2} \frac{1}{\psi_{k}}\left(1+\frac{1}{\psi_{k}}\right) \sigma_{Y}^{2}\right)-r_{t}
$$

Observe that the term inside the min operator in (64) is equal to $\hat{k}_{k}$ in Equation (61), which is the long-term yield in a homogeneous agent economy where Agent $k$ is the representative agent (see also Equation (A31) in the appendix). The long-term yield consists of $\beta_{k}$, the rate of impatience; $\frac{1}{\psi_{k}} \mu_{Y, k}$, the growth rate of aggregate endowments scaled by the inverse of the elasticity of intertemporal substitution parameter; $-\frac{1}{2} \frac{1}{\psi_{k}}\left(1+\frac{1}{\psi_{k}}\right) \sigma_{Y}^{2}$, the term that adjusts for precautionary savings determined by the elasticity of intertemporal substitution parameter, and $\mu_{Y, k}$, the growth rate of $Y$ expected by investor $k$. Together, these terms give the "discount rate" used by Agent $k$ for valuing risky cashflows. One can interpret similarly the term inside the min operator in (65): because this is the yield on a discount bond with a single terminal payoff that is riskless, compared to the expression in (64), the terms that adjust for risk and for growth are missing.

Proposition 9 implies that the long-term yield will be set by whichever agent has the lower discount rate, and not necessarily the agent who survives $\mathbb{P}$-almost surely in the long run; see Kogan, Ross, Wang, and Westerfield (2006). The intuition is that even though an agent may not survive in the long-run in the almost-surely sense, she may still be the dominant agent in rare states of the world, which are also high marginal utility states for this investor, and thus important for asset prices.

Corollary 5 (Long-term yield when one agent has correct beliefs) Suppose that agents have identical preferences (that is, $\beta_{1}=\beta_{2}=\beta, \gamma_{1}=\gamma_{2}=\gamma, \psi_{1}=\psi_{2}=\psi$ ), and Agent 1 has correct beliefs $\left(\mu_{Y, 1}=\mu_{Y}\right)$, whereas Agent 2 has incorrect beliefs about the expected growth rate of the 
economy. The long-term yield, $y_{T-t}^{Y}$, is set by Agent 2 if and only if (i) $\mu_{Y, 2}<\mu_{Y}$ and $\psi<1$, or (ii) $\mu_{Y, 2}>\mu_{Y}$ and $\psi>1$.

\subsection{The Equity Risk Premium and Volatility of Stock-Market Returns}

The price of the stock, which can be interpreted as the market portfolio, and its cumulative return, $R_{t}$, which consists of capital gains plus dividends, is described by the process:

$$
\frac{d P_{t}^{Y}+Y_{t} d t}{P_{t}^{Y}}=d R_{t}=\mu_{R, t} d t+\sigma_{R, t} d Z_{t}
$$

From Equation (24), we see that once we have the value of the spanning assets, one can obtain the price of equity by integrating the value of these spanning assets with respect to their maturities. The risk premium on equity, which pays $Y_{t}$ in perpetuity, is given by the standard asset pricing equation:

$$
E_{t}\left[\frac{d P_{t}^{Y}+Y_{t} d t}{P_{t}^{Y}}-r_{t} d t\right]=-E_{t}\left[\frac{d \pi_{t}}{\pi_{t}} \frac{d P_{t}^{Y}}{P_{t}^{Y}}\right]
$$

Applying Ito's Lemma to $P_{t}^{Y}=Y_{t} p_{t}^{Y}$ and using Equation (66) leads to the following proposition.

Proposition 10 (Volatility of stock market returns and equity risk premium) The volatility of stock market returns, $\sigma_{R, t}^{Y}$, is

$$
\sigma_{R, t}^{Y}=\sigma_{Y}+\frac{1}{p_{t}^{Y}}\left(\frac{\partial p_{t}^{Y}}{\partial \omega_{t}} \sigma_{Y}+\frac{\partial p_{t}^{Y}}{\partial \nu_{1, t}} \nu_{1, t} \sigma_{\nu_{1}, t}\right)
$$

the risk premium on equity is

$$
\mu_{R, t}^{Y}-r_{t}=\theta_{t} \sigma_{R, t}^{Y}=\left(\mathbf{R}_{t} \sigma_{Y}+\left[\frac{\mu_{Y}-\boldsymbol{\mu}_{Y, t}}{\sigma_{Y}}\right]\right) \sigma_{R, t}^{Y}
$$

and, Agent $k$ 's perception of the risk premium is given by

$$
\mu_{R, k, t}^{Y}-r_{t}=\left(\mathbf{R}_{t} \sigma_{Y}+\left[\frac{\mu_{Y, k}-\boldsymbol{\mu}_{Y, t}}{\sigma_{Y}}\right]\right) \sigma_{R, t}^{Y}
$$

In a model with a single representative investor, stock return volatility, $\sigma_{R, t}$ is equal to fundamental volatility, $\sigma_{Y}$. From (67) we see that in a model with heterogeneous investors, stock 
market return volatility is the sum of fundamental volatility, $\sigma_{Y}$, and excess volatility, which is given by the second term: $\frac{1}{p_{t}^{Y}}\left(\frac{\partial p_{t}^{Y}}{\partial \omega_{t}} \sigma_{Y}+\frac{\partial p_{t}^{Y}}{\partial \nu_{1, t}} \nu_{1, t} \sigma_{\nu_{1}, t}\right)$. This second term depends on fluctuations in the price-dividend ratio. The excess-volatility term can be interpreted as the elasticity of the price-dividend ratio with respect to habit multiplied by the volatility of habit, plus the elasticity of the price-dividend ratio with respect to the consumption share multiplied by the volatility of the consumption share. When demand for precautionary savings is not too large, the price-dividend ratio is monotonic and countercyclical, and so excess volatility is positive, as in the data.

We now discuss the equity risk premium. From Proposition 10, we see that while agents agree on conditional stock return volatility, they may disagree on the conditional risk premium; that is, each agent will have her own perception of the equity risk premium, which is given by the expression in Equation (69). The central planner's view of the conditional risk premium, which is given in Equation (68), is the product of the market price of risk, $\theta_{t}$, and the volatility of stock market returns, $\sigma_{R, t}^{Y}$. The risk premium will be high when: (i) in aggregate, agents are pessimistic, $\boldsymbol{\mu}_{Y, t}<\mu_{Y}$; (ii) the aggregate risk aversion in the economy, $\mathbf{R}_{t}$, is high; and (iii) stock return volatility, $\sigma_{R, t}^{Y}$, is high. Quantitatively, the first and third channels are the most important for generating a risk premium that is high relative to the risk premium in an economy where agents are homogeneous. ${ }^{22}$

Heterogeneity in either risk aversion or beliefs leads to cyclicality in the price of risk and stock market return volatility, which feeds into the risk premium, whereas heterogeneity in $\psi$ or the rate of of time preference has no such impact.

In the data, the price of risk, stock market return volatility and the conditional risk premium are all countercyclical and this can be generated by either heterogeneity in risk aversion or heterogeneity in beliefs.

\section{General Affine Model}

In this section, we show how to price financial assets when the logarithm of aggregate endowment and agents' beliefs follow general affine processes, instead of the particular processes assumed in Equations (35) and (37).

\footnotetext{
${ }^{22}$ Note that if stock return volatility, $\sigma_{R, t}^{Y}$, is higher than fundamental volatility, $\sigma_{Y}$, the risk premium can be higher than in either of the two homogeneous agent economies.
} 
We start by recalling that an affine process $V_{t}$ with some state space $D \subset \mathbb{R}^{d}$ is defined as a Markov process whose conditional characteristic function is of the form, for any $a \in \mathbb{R}^{d}$,

$$
E_{t}\left[e^{i a \cdot V_{u}}\right]=e^{\chi_{1}(t, u, z)+\chi_{2}(t, u, z) \cdot V_{t}}
$$

for some coefficients $\chi_{1}(t, u, z)$ and $\chi_{2}(t, u, z)$. We also observe that if $V_{t}$ is analytic affine, then $E_{t}\left[e^{i a \cdot V_{u}}\right]$, where each component of $a$ is in $\mathbb{C}$, is a holomorphic function whose restriction to the real numbers is real-valued. The class of analytic affine processes includes the class of affine jump diffusions in Duffie, Pan, and Singleton (2000).

Suppose $y=\ln Y$ is given by an analytic affine process with drift and diffusion driven by analytic affine processes. It then follows from (12) that

$$
\hat{\pi}_{k, t}=e^{\varsigma_{k, t}}
$$

where $\varsigma_{k, t}$ is an analytic affine process, given by

$$
\varsigma_{k, t}=\ln \left(\lambda_{k, 0} \xi_{k, t}\right)-\beta_{k} t-\left(\gamma_{k}-\frac{1}{\psi_{k}}\right) \omega_{t}-\frac{1}{\psi_{k}} y_{t} .
$$

Consider a cashflow process, $X_{t}=e^{x_{t}}$, where $x_{t}$ is analytic affine. The moment generating function

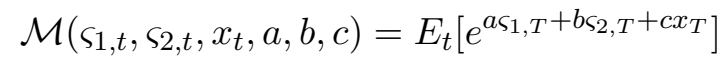

can be evaluated explicitly, because $\varsigma_{1, T}, \varsigma_{2, T}$ and $x_{T}$ are jointly affine. The following proposition shows how one can obtain asset prices using the moment generating function, when the logarithm of aggregate endowment and agents' beliefs follow general analytic affine processes.

Proposition 11 (Values of $\phi_{\boldsymbol{n}, \mathbf{1}, t, \boldsymbol{u}}^{\boldsymbol{X}}$ and $\phi_{\boldsymbol{n}, \mathbf{2}, \boldsymbol{t}, \boldsymbol{u}}^{\boldsymbol{X}}$ for general affine processes) If the logarithm of aggregate endowment and agents' beliefs follow general analytic affine processes, then the values of $\phi_{n, 1, t, u}^{X}$ and $\phi_{n, 2, t, u}^{X}$ that pay $X_{u}$ at $u$ whenever $\frac{\hat{\pi}_{1, u}}{\hat{\pi}_{2, u}}>R$ and $\frac{\hat{\pi}_{1, u}}{\hat{\pi}_{2, u}}<R$, respectively, are:

$$
\begin{aligned}
\phi_{n, 1, t, u} & =e^{-\left[\left(1-\frac{n}{\gamma_{1}}\right) \varsigma_{1, t}+\frac{n}{\gamma_{2}} \varsigma_{2, t}\right]-x_{t}}\left[\frac{1}{\pi} \int_{-\infty}^{\infty} e^{i \rho z} \frac{1}{z} \operatorname{Im}\left[\mathcal{M}\left(\varsigma_{1, t}, \varsigma_{2, t}, x_{t}, u-t,\left(1-\frac{n}{\gamma_{1}}\right)+i z, \frac{n}{\gamma_{2}}-i z, 1\right)\right] d z\right. \\
& \left.+\frac{1}{2} \mathcal{M}\left(\varsigma_{1, t}, \varsigma_{2, t}, x_{t}, u-t, 1-\frac{n}{\gamma_{1}}, \frac{n}{\gamma_{2}}, 1\right)\right]
\end{aligned}
$$

and

$\phi_{n, 2, t, u}=e^{-\left[\frac{n}{\gamma_{1}} \varsigma_{1, t}+\left(1-\frac{n}{\gamma_{2}}\right) \varsigma_{2, t}\right]-x_{t}}\left[-\frac{1}{\pi} \int_{-\infty}^{\infty} e^{i \rho z} \frac{1}{z} \operatorname{Im}\left[\mathcal{M}\left(\varsigma_{1, t}, \varsigma_{2, t}, x_{t}, u-t, \frac{n}{\gamma_{1}}+i z, 1-\frac{n}{\gamma_{2}}-i z, 1\right)\right] d z\right.$ 


$$
\left.+\frac{1}{2} \mathcal{M}\left(\varsigma_{1, t}, \varsigma_{2, t}, x_{t}, u-t, \frac{n}{\gamma_{1}}, 1-\frac{n}{\gamma_{2}}, 1\right)\right] .
$$

\section{Conclusion}

In this paper, we study an endowment economy where there are two types of agents, each with "catching up with the Joneses" utility. The two agents are heterogeneous with respect to their preference parameters for the subjective rate of time preference, relative risk aversion, and sensitivity to habit, and also with respect to their beliefs.

Our main contribution is to solve in closed form for the equilibrium in this economy and to identify the optimal consumption-sharing rule, without restricting the risk aversions of the two agents to particular values. We use this closed-form solution to identify the state price density, market price of risk, the locally risk free interest rate the stock price, the equity market risk premium, the volatility of stock returns, and the term structure of interest rates. We also derive explicitly the condition for the model to be stationary, in the sense that both types of agents survive in the long run. We then analyze how heterogeneity in preferences and beliefs affects the properties of asset returns.

We find that heterogeneity in beliefs about the growth rate of aggregate endowment and heterogeneity in preferences are capable of generating a market price of risk of that is substantially higher than that in a homogeneous-agent economy. Moreover, heterogeneity in preferences and beliefs increase stock-return volatility by as much as two to four times the fundamental volatility of aggregate endowment. Consequently, the equity risk premium, which is the product of the market price of risk and stock return volatility, is considerably higher in a model where both beliefs and preferences are heterogeneous, and this is accompanied neither by an increase in the level of the short-term riskless rate, nor an increase in its volatility. The aggregate risk aversion in the heterogeneous-agent economy is countercyclical, and consequently, the model is consistent also with several dynamic properties of asset prices; for example, when aggregate consumption falls, expected stock returns, stock-return volatility, and the market price of risk rise, and price-dividend ratios decline. 
The methodology we develop to identify the consumption-sharing rule for agents who have both heterogeneous priors and heterogeneous preferences is sufficiently general that, as long as markets are complete, it can be applied to models set in discrete or continuous time, to endowment processes that are in the exponential affine jump-diffusion class, and, to settings with arbitrary updating of beliefs. 


\section{A Appendix: Lagrange's Theorem and Proofs for Propositions and Corollaries}

We begin by stating a number of definitions and theorems from complex analysis that are used to derive results in the paper. In particular, the insight from Lagrange that is central to the analysis in the paper is given in Theorem A2.

Definition A1 If $U$ is an open subset of $\mathbb{C}$ and $f: U \rightarrow \mathbb{C}$ is a complex function on $U$, we say that $f$ is complex differentiable at a point $z_{0}$ of $U$ if the limit

$$
f^{\prime}\left(z_{0}\right)=\lim _{z \rightarrow z_{0}} \frac{f(z)-f\left(z_{0}\right)}{z-z_{0}}
$$

exists. The limit here is taken over all sequences of complex numbers approaching $z_{0}$, and for all such sequences the difference quotient has to approach the same number $f^{\prime}\left(z_{0}\right)$.

Definition A2 If $f$ is complex differentiable at every point $z_{0}$ in $U$, we say that $f$ is holomorphic on $U$. We say that $f$ is holomorphic at the point $z_{0}$ if it is holomorphic on some neighborhood of $z_{0}$. We say that $f$ is holomorphic on some non-open set $A$ if it is holomorphic in an open set containing A.

Definition A3 A function $f$ is complex analytic on an open set $D$ in the complex plane if for any $z_{0}$ in $D$ one can write

$$
f(z)=\sum_{n=0}^{\infty} a_{n}\left(z-z_{0}\right)^{n},
$$

in which the coefficients $a_{0}, a_{1}, \ldots$ are complex numbers and the series is convergent for $z$ in $a$ neighborhood of $z_{0}$.

Theorem A1 (Complex Analytic) A function $f$ is complex analytic on an open set $D$ in the complex plane if and only if it is holomorphic in $D$.

We are now ready to state the theorem that allows us to find closed-form series expansions for the sharing rule and complex analytic functions of the sharing rule.

Theorem A2 (Lagrange) Suppose the dependence between the variables $w$ and $z$ is implicitly defined by an equation of the form

$$
w=f(z),
$$

where $f$ is complex analytic in a neighborhood of 0 and $f^{\prime}(0) \neq 0$. Then for any function $g$ which is complex analytic in a neighborhood of 0 ,

$$
g(z)=g(0)+\sum_{n=1}^{\infty} \frac{w^{n}}{n !}\left[\frac{d^{n-1}}{d x^{n-1}} g^{\prime}(x)\left[\varphi(x)^{n}\right]\right]_{x=0},
$$

where $\varphi(z)=\frac{z}{f(z)}$. 
Note that the above theorem does not provide a radius of convergence for the series in Equation (A1). While the original proof of Theorem A2 due to Lagrange is not very straightforward, a relatively easier proof can be obtained by using Cauchy's Integral Formula.

\section{A.1 Proof of Proposition 1: Consumption-sharing rule}

Equation (13) is equivalent to

$$
A_{t}\left(1-\nu_{1, t}\right)^{\eta}=\nu_{1, t}
$$

which implicitly defines $\nu_{1, t}$ in terms of $A_{t}$. To solve explicitly for $\nu_{1, t}$, we apply Theorem A2, expanding around the point $\nu_{1, t}=0$, with

$$
\begin{aligned}
& f(z)=z(1-z)^{-\eta}, \\
& \varphi(z)=(1-z)^{\eta}, \\
& g(z)=z,
\end{aligned}
$$

after showing that $f$ is complex analytic in some neighborhood of 0 . We know from the binomial series expansion that for $z \in \mathbb{C}$, such that $|z|<1$,

$$
(1-z)^{-\eta}=\sum_{n=0}^{\infty}\left(\begin{array}{c}
-\eta \\
k
\end{array}\right)(-)^{n} z^{n}
$$

where $\left(\begin{array}{c}-\eta \\ k\end{array}\right)=\Pi_{j=1}^{k} \frac{-\eta-k+j}{j}$ is the generalized binomial coefficient. Therefore, $(1-z)^{-\eta}$ is complex analytic in the open ball $\{z \in \mathbb{C}:|z|<1\}$. Since $z$ is complex analytic for all $z \in \mathbb{C}$, it follows that $f$ as defined in (A2) is complex analytic in the open ball $\{z \in \mathbb{C}:|z|<1\}$. It therefore follows from Theorem A2 that

$$
\nu_{1, t}=\sum_{n=1}^{\infty} \frac{A_{t}^{n}}{n !} \frac{d^{n-1}}{d x^{n-1}}\left[(1-x)^{\eta n}\right]_{x=0}
$$

Since

$$
\frac{d^{n-1}}{d x^{n-1}}\left[(1-x)^{n \eta}\right]=(-)^{n-1} \eta n(\eta n-1)(\eta n-2) \ldots(\eta n-(n-2))(1-x)^{\eta n-(n-1)}
$$

it follows that

$$
\begin{aligned}
& \nu_{1, t}=-\sum_{n=1}^{\infty} \frac{\left(-A_{t}\right)^{n}}{n}\left(\begin{array}{c}
\eta n \\
n-1
\end{array}\right), \\
& \nu_{2, t}=1+\sum_{n=1}^{\infty} \frac{\left(-A_{t}\right)^{n}}{n}\left(\begin{array}{c}
\eta n \\
n-1
\end{array}\right) .
\end{aligned}
$$

We shall now determine the radius of convergence of the above series. From d'Alembert's ratio test, it follows that the above series converge absolutely for all $A \in \mathbb{C}$ s.t. $|A|<\bar{R}$, where

$$
\bar{R}=\lim _{n \rightarrow \infty} \frac{n+1}{n}\left|\frac{\left(\begin{array}{c}
\eta n \\
n-1
\end{array}\right)}{\left(\begin{array}{c}
\eta(n+1) \\
n
\end{array}\right)}\right| .
$$


We wish to evaluate the above limit for all $\eta \in \mathbb{R}$ such that $\eta>1$. Hence, $\left(\begin{array}{c}\eta n \\ n-1\end{array}\right)$ and $\left(\begin{array}{c}\eta(n+1) \\ n\end{array}\right)$ are positive and real, and so

$$
\bar{R}=\lim _{n \rightarrow \infty} \frac{n+1}{n} \frac{\left(\begin{array}{c}
\eta n \\
n-1
\end{array}\right)}{\left(\begin{array}{c}
\eta(n+1) \\
n
\end{array}\right)} .
$$

We note that the generalized binomial coefficient, $\left(\begin{array}{l}z \\ k\end{array}\right)=\Pi_{j=1}^{k} \frac{z-k+j}{j}$, can be written as

$$
\left(\begin{array}{l}
z \\
k
\end{array}\right)=\frac{\Gamma(z+1)}{\Gamma(z-k+1) \Gamma(k+1)}
$$

where $\Gamma(z)$ is the Gamma function, which for $\Re(z)>0$ (where $\Re(z)$ denotes the real part of $z$ ), has the integral representation,

$$
\Gamma(z)=\int_{0}^{\infty} t^{z-1} e^{-t} d t
$$

The Euler Beta function, $B(x, y)$, defined by

$$
B(x, y)=\int_{0}^{1} t^{x-1}(1-t)^{y-1} d t
$$

can be written in terms of the Gamma function as follows,

$$
B(x, y)=\frac{\Gamma(x) \Gamma(y)}{\Gamma(x+y)} .
$$

Together with (A5), the above expression implies that the generalized binomial coefficient is given by

$$
\left(\begin{array}{l}
z \\
k
\end{array}\right)=\frac{1}{(z+1) B(z-k+1, k+1)}
$$

Hence,

$$
\bar{R}=\lim _{n \rightarrow \infty} \frac{n+1}{n} \frac{\eta(n+1)+1}{\eta n+1} \frac{B((\eta-1)(n+1), n+1)}{B((\eta-1) n, n)} .
$$

To evaluate the above limit, we start by recalling Stirling's series for the Gamma function

$$
\Gamma(z)=\sqrt{2 \pi} e^{-z} z^{z-\frac{1}{2}}\left(1+O\left(\frac{1}{z}\right)\right),
$$

which together with (A6) implies that

$$
\bar{R}=\lim _{n \rightarrow \infty} \frac{n+1}{n} \frac{\eta(n+1)+1}{\eta n+1} \frac{\frac{((\eta-1)(n+1))^{(\eta-1)(n+1)-\frac{1}{2}}(n+1)^{(n+1)-\frac{1}{2}}}{((\eta-1)(n+1)+(n+1))^{((\eta-1)(n+1)+(n+1))-\frac{1}{2}}}}{\frac{((\eta-1) n)^{((\eta-1) n)-\frac{1}{2}} n^{n-\frac{1}{2}}}{(((\eta-1) n)+n)^{(((\eta-1) n)+n)-\frac{1}{2}}}}
$$

Simplifying the above expression gives

$$
\bar{R}=\lim _{n \rightarrow \infty} \frac{n+1}{n} \frac{\eta(n+1)+1}{\eta n+1} \frac{(\eta-1)^{\eta-1}}{\eta^{\eta}} \sqrt{\frac{n}{n+1}}
$$




$$
=\frac{(\eta-1)^{\eta-1}}{\eta^{\eta}}
$$

Since $A_{t}$ is a geometric Brownian motion, it is positive and real. Hence, the right-hand side of (A4) is absolutely convergent for $A_{t}<\frac{(\eta-1)^{\eta-1}}{\eta^{\eta}}$.

We now derive a series expansion for $\nu_{2, t}$ in terms of $A_{t}$, which is absolutely convergent for $A_{t}>\frac{(\eta-1)^{\eta-1}}{\eta^{\eta}}$. We start by rearranging (13) to obtain

$$
\nu_{2, t}=A_{t}^{-1 / \eta}\left(1-\nu_{2, t}\right)^{1 / \eta}
$$

To find $\nu_{2, t}$, we apply Theorem A2, expanding around the point $\nu_{2, t}=0$, with $f, \varphi$ and $g$, defined as below

$$
\begin{aligned}
& f(z)=z(1-z)^{-1 / \eta} \\
& \varphi(z)=(1-z)^{1 / \eta} \\
& g(z)=z
\end{aligned}
$$

We can show that our newly defined $f$ is complex analytic in the open ball, $\{z \in \mathbb{C}:|z|<1\}$, in the same way as for (A2). Hence, Theorem A2 implies that

$$
\nu_{2, t}=\sum_{n=1}^{\infty} \frac{\left(A_{t}^{-1 / \eta}\right)^{n}}{n !} \frac{d^{n-1}}{d x^{n-1}}\left[(1-x)^{n / \eta}\right]_{x=0} .
$$

Because

$$
\frac{d^{n-1}}{d x^{n-1}}\left[(1-x)^{n / \eta}\right]=(-)^{n-1} \frac{n}{\eta}\left(\frac{n}{\eta}-1\right)\left(\frac{n}{\eta}-2\right) \ldots\left(\frac{n}{\eta}-(n-2)\right)(1-x)^{\frac{n}{\eta}-(n-1)},
$$

it follows that

$$
\nu_{2, t}=-\sum_{n=1}^{\infty} \frac{\left(-A_{t}^{-\frac{1}{\eta}}\right)^{n}}{n}\left(\begin{array}{c}
\frac{n}{\eta} \\
n-1
\end{array}\right)=\sum_{n=1}^{\infty} \frac{(-)^{n-1}\left(A_{t}^{-\frac{1}{\eta}}\right)^{n}}{n}\left(\begin{array}{c}
\frac{n}{\eta} \\
n-1
\end{array}\right) .
$$

By comparing the above expression with (A.1), we can see that (A.1) is absolutely convergent if $A_{t}^{-1 / \eta}<\frac{\left(\frac{1}{\eta}-1\right)^{\frac{1}{\eta}-1}}{\frac{1}{\eta} \frac{1}{\eta}}$, that is, if $A_{t}>\frac{(\eta-1)^{\eta-1}}{\eta^{\eta}}$. To summarize, we have

$$
\nu_{2, t}=\left\{\begin{array}{cl}
-\sum_{n=1}^{\infty} \frac{\left(-A_{t}^{-\frac{1}{\eta}}\right)^{n}}{n}\left(\begin{array}{c}
\frac{n}{\eta} \\
n-1
\end{array}\right) & , A_{t}>\bar{R} \\
1+\sum_{n=1}^{\infty} \frac{\left(-A_{t}\right)^{n}}{n}\left(\begin{array}{c}
n \eta \\
n-1
\end{array}\right) & , A_{t}<\bar{R}
\end{array}\right.
$$

where $\bar{R}$ is given in (A.1). Using (3.1) we can write the expressions for the sharing rule as (14). 


\section{A.2 Proof of Proposition 2: State-price density}

The equilibrium state price density is given by (45). To find a closed-form expression for the equilibrium state-price density, we find series expansions for $\nu_{k, t}^{-\gamma_{k}}, k \in\{1,2\}$. To find a series expansion for $\nu_{2, t}^{-\gamma_{2}}$, note that

$$
\nu_{2, t}^{-\gamma_{2}}=\left(1-\nu_{1, t}\right)^{-\gamma_{2}},
$$

and use Theorem A2 to expand around the point $\nu_{1, t}=0$. To do this we define

$$
g(z)=(1-z)^{-\gamma_{2}}
$$

which is complex analytic in the open ball $\{z \in \mathbb{C}:|z|<1\}$. Hence, with $f$ and $\varphi$ defined as in (A2) and (A3), respectively, Theorem A2 implies that

$$
\begin{aligned}
g\left(\nu_{1, t}\right) & =\left(1-\nu_{1, t}\right)^{-\gamma_{2}} \\
& =g(0)+\sum_{n=1}^{\infty} \frac{A_{t}^{n}}{n !} \frac{d^{n-1}}{d x^{n-1}}\left[g^{\prime}(x) \varphi(x)^{n}\right]_{x=0} \\
& =1+\sum_{n=1}^{\infty} \frac{A_{t}^{n}}{n !} \frac{d^{n-1}}{d x^{n-1}}\left[\gamma_{2}(1-x)^{n \eta-\gamma_{2}-1}\right]_{x=0} .
\end{aligned}
$$

Since,

$$
\begin{aligned}
& \frac{d^{n-1}}{d x^{n-1}} \gamma_{2}(1-x)^{n \eta-\gamma_{2}-1} \\
& =\gamma_{2}(-)^{n-1}\left(n \eta-\gamma_{2}-1\right)\left(n \eta-\gamma_{2}-2\right) \ldots\left(n \eta-\gamma_{2}-(n-1)\right)(1-x)^{n \eta-\gamma_{2}-(n-1)},
\end{aligned}
$$

it follows that

$$
\nu_{2, t}^{-\gamma_{2}}=1-\gamma_{2} \sum_{n=1}^{\infty} \frac{\left(-A_{t}\right)^{n}}{n}\left(\begin{array}{c}
n \eta-\gamma_{2}-1 \\
n-1
\end{array}\right) .
$$

D'Alembert's ratio test implies that the above series converges absolutely for all $A \in \mathbb{C}$ such that $|A|<\bar{R}$, where

$$
\bar{R}=\lim _{n \rightarrow \infty} \frac{n+1}{n} \frac{\left(\begin{array}{c}
\eta n-\gamma_{2}-1 \\
n-1
\end{array}\right)}{\left(\begin{array}{c}
\eta(n+1)-\gamma_{2}-1 \\
n
\end{array}\right)} .
$$

Using (A7), we rewrite the above expression as

$$
\bar{R}=\lim _{n \rightarrow \infty} \frac{n+1}{n} \frac{\eta(n+1)-\gamma_{2}}{\eta n-\gamma_{2}} \frac{B\left((\eta-1)(n+1)-\gamma_{2}-1, n+1\right)}{B\left((\eta-1) n-\gamma_{2}-1, n\right)} .
$$

Hence, using (A6) and (A8), we obtain

$$
\bar{R}=\lim _{n \rightarrow \infty} \frac{n+1}{n} \frac{\eta(n+1)-\gamma_{2}}{\eta n-\gamma_{2}} \frac{\frac{\left[(\eta-1)(n+1)-\left(1+\gamma_{2}\right)\right]^{(\eta-1)(n+1)-\left(1+\gamma_{2}\right)-1 / 2}(n+1)^{n+1-1 / 2}}{\left[\eta(n+1)-\left(1+\gamma_{2}\right)\right]^{\eta(n+1)-\left(1+\gamma_{2}\right)-1 / 2}}}{\frac{\left[(\eta-1) n-\left(1+\gamma_{2}\right)\right]^{(\eta-1) n-\left(1+\gamma_{2}\right)-1 / 2} n^{n-1 / 2}}{\left[\eta n-\left(1+\gamma_{2}\right)\right]^{\eta n-\left(1+\gamma_{2}\right)-1 / 2}}}
$$


Simplifying the above expression gives

$$
\bar{R}=\frac{(\eta-1)^{(\eta-1)}}{\eta^{\eta}}
$$

Since $A_{t}$ is a geometric Brownian motion, $A_{t}$ is real and positive, and so the right-hand side of (A11) is absolutely convergent if $A_{t}<\frac{(\eta-1)^{(\eta-1)}}{\eta^{\eta}}=\bar{R}$. Hence,

$$
\nu_{2, t}^{-\gamma_{2}}=1-\gamma_{2} \sum_{n=1}^{\infty} \frac{\left(-A_{t}\right)^{n}}{n}\left(\begin{array}{c}
n \eta-\gamma_{2}-1 \\
n-1
\end{array}\right), A_{t}<\bar{R} .
$$

Using (12) and (15), we can rewrite the above expression as

$$
\nu_{2, t}^{-\gamma_{2}}=\sum_{n=0}^{\infty} a_{n, 2}^{\pi}\left(\frac{\hat{\pi}_{1, t}}{\hat{\pi}_{2, t}}\right)^{\frac{n}{\gamma_{1}}}, \frac{\hat{\pi}_{1, t}}{\hat{\pi}_{2, t}}<\frac{\gamma_{1}^{\gamma_{2}}}{\gamma_{2}^{\gamma_{2}}}\left(\frac{\gamma_{2}}{\gamma_{1}}-1\right)^{\gamma_{2}-\gamma_{1}}
$$

where $a_{n, 2}^{\pi}$ is defined in (18). Therefore, the equilibrium state-price density is given by

$$
\pi_{t}=\sum_{n=0}^{\infty} a_{n, 2}^{\pi} \hat{\pi}_{1, t}^{\frac{n}{\gamma_{1}}} \hat{\pi}_{2, t}^{1-\frac{n}{\gamma_{1}}}, \frac{\hat{\pi}_{1, t}}{\hat{\pi}_{2, t}}<\frac{\gamma_{1}^{\gamma_{2}}}{\gamma_{2}^{\gamma_{2}}}\left(\frac{\gamma_{2}}{\gamma_{1}}-1\right)^{\gamma_{2}-\gamma_{1}} .
$$

To find an expression for the state-price density when $A_{t}>\frac{(\eta-1)^{(\eta-1)}}{\eta^{\eta}}$, we find a series expansion for $\nu_{1, t}^{-\gamma_{1}}$, which is absolutely convergent for $A_{t}>\frac{(\eta-1)^{(\eta-1)}}{\eta^{\eta}}$. Note that

$$
\nu_{1, t}^{-\gamma_{1}}=\left(1-\nu_{2, t}\right)^{-\gamma_{1}},
$$

and use Theorem A2 to expand around the point $\nu_{2, t}=0$. To do this, we define

$$
g(z)=(1-z)^{-\gamma_{1}}
$$

which is complex analytic in the open ball $\{z \in \mathbb{C}:|z|<1\}$. Hence, with $f$ and $\varphi$ defined as in (A9) and (A10), respectively, Theorem A2 implies that

$$
\begin{aligned}
g\left(\nu_{2, t}\right) & =\left(1-\nu_{2, t}\right)^{-\gamma_{1}} \\
& =g(0)+\sum_{n=1}^{\infty} \frac{\left(A_{t}^{-1 / \eta}\right)^{n}}{n !} \frac{d^{n-1}}{d x^{n-1}}\left[g^{\prime}(x) \varphi(x)^{n}\right]_{x=0} \\
& =1+\sum_{n=1}^{\infty} \frac{\left(A_{t}^{-1 / \eta}\right)^{n}}{n !} \frac{d^{n-1}}{d x^{n-1}}\left[\gamma_{1}(1-x)^{\frac{n}{\eta}-\gamma_{1}-1}\right]_{x=0} .
\end{aligned}
$$

Because,

$$
\frac{d^{n-1}}{d x^{n-1}}\left[\gamma_{1}(1-x)^{\frac{n}{\eta}-\gamma_{1}-1}\right]=
$$




$$
\gamma_{1}(-)^{n-1}\left(\frac{n}{\eta}-\gamma_{1}-1\right)\left(\frac{n}{\eta}-\gamma_{1}-2\right) \ldots\left(\frac{n}{\eta}-\gamma_{1}-(n-1)\right)(1-x)^{\frac{n}{\eta}-\gamma_{1}-(n-1)},
$$

it follows that

$$
\nu_{1, t}^{-\gamma_{1}}=1-\gamma_{1} \sum_{n=1}^{\infty} \frac{\left(-A_{t}^{-1 / \eta}\right)^{n}}{n}\left(\begin{array}{c}
\frac{n}{\eta}-\gamma_{1}-1 \\
n-1
\end{array}\right) .
$$

By comparing the above expression with (A11), we can see that (A13) is absolutely convergent if $A_{t}^{-1 / \eta}<\frac{\left(\frac{1}{\eta}-1\right)^{\frac{1}{\eta}-1}}{\frac{1}{\eta} \frac{1}{\eta}}$, i.e. if $A_{t}>\frac{(\eta-1)^{\eta-1}}{\eta^{\eta}}=\bar{R}$. Thus,

$$
\nu_{1, t}^{-\gamma_{1}}=1-\gamma_{1} \sum_{n=1}^{\infty} \frac{\left(-A_{t}^{-1 / \eta}\right)^{n}}{n}\left(\begin{array}{c}
\frac{n}{\eta}-\gamma_{1}-1 \\
n-1
\end{array}\right), A_{t}>\bar{R} .
$$

Using (12) and (15), we can rewrite the above expression as

$$
\nu_{1, t}^{-\gamma_{1}}=\sum_{n=0}^{\infty} a_{n, 1}^{\pi}\left(\frac{\hat{\pi}_{2, t}}{\hat{\pi}_{1, t}}\right)^{\frac{n}{\gamma_{2}}}, \frac{\hat{\pi}_{1, t}}{\hat{\pi}_{2, t}}>\frac{\gamma_{1}^{\gamma_{2}}}{\gamma_{2}^{\gamma_{2}}}\left(\frac{\gamma_{2}}{\gamma_{1}}-1\right)^{\gamma_{2}-\gamma_{1}}
$$

where $a_{n, 1}^{\pi}$ is defined in (17). Therefore, the equilibrium state-price density is given by

$$
\pi_{t}=\sum_{n=0}^{\infty} a_{n, 2}^{\pi} \hat{\pi}_{1, t}^{\frac{n}{\gamma_{1}}} \hat{\pi}_{2, t}^{1-\frac{n}{\gamma_{1}}}, \frac{\hat{\pi}_{1, t}}{\hat{\pi}_{2, t}}>\frac{\gamma_{1}^{\gamma_{2}}}{\gamma_{2}^{\gamma_{2}}}\left(\frac{\gamma_{2}}{\gamma_{1}}-1\right)^{\gamma_{2}-\gamma_{1}} .
$$

The expressions in (16) follow from (A12) and (A14).

\section{A.3 Proof of Corollary 1: State-price density under identical risk aversion}

First we note that

$$
\lim _{a \rightarrow 0}\left(\frac{\gamma+a}{\gamma}-1\right)^{a}=1
$$

Therefore, setting $\gamma_{1}=\gamma_{2}=\gamma$ implies that

$$
\frac{\gamma_{1}^{\gamma_{2}}}{\gamma_{2}^{\gamma_{2}}}\left(\frac{\gamma_{2}}{\gamma_{1}}-1\right)^{\gamma_{2}-\gamma_{1}}=1
$$

Also note that after some tedious algebra, we can show that

$$
\gamma\left(\begin{array}{c}
n-\gamma-1 \\
n
\end{array}\right) \frac{(-)^{n+1}}{n}=\left(\begin{array}{l}
\gamma \\
n
\end{array}\right)
$$

Therefore, when $\gamma_{1}=\gamma_{2}=\gamma,(17)$ and (18) reduce to (20).

The expression in (22) is obtained from (19) by using Newton's Binomial Theorem (for nonintegral powers). When $\gamma$ is a natural number, the expression in (22) can be obtained also from (21) by using the Binomial Theorem for integral powers, and one could obtain (22) also directly from the first-order condition for consumption in (11). 


\section{A.4 Proof of Proposition 3: Price of dividend strip}

Rather than considering a dividend strip based where the aggregate endowment, $Y$, is the dividend, we shall derive results for a more general dividend strip, which pays out $X_{u}$ at date $u$, where the evolution of $X$ is given by

$$
\frac{d X_{t}}{X_{t}}=\mu_{X} d t+\sigma_{X}^{s y s} d Z_{t}+\sigma_{X}^{i d} d Z_{t}^{i d}
$$

where $Z_{t}^{i d}$ is a standard Brownian motion under $\mathbb{P}$, orthogonal to $Z_{t}$, and

$$
\frac{\mu_{Y, k}-\mu_{Y}}{\sigma_{Y}}=\frac{\mu_{X, k}-\mu_{X}}{\sigma_{X}^{s y s}}
$$

The date- $t$ price of the dividend strip which pays out $X_{u}$ at date $u>t$ is denoted by $V_{t, u}^{X}$, where

$$
V_{t, u}^{X}=v_{t, u}^{X} X_{t},
$$

and

$$
v_{t, u}^{X}=E_{t} \int_{t}^{\infty}\left[\frac{\pi_{u}}{\pi_{t}} \frac{X_{u}}{X_{t}} d t\right]
$$

We shall derive an expression for $v_{t, u}^{X}$, and then, to get the equations in the proposition giving the price of a dividend strip where the endowment is the dividend, we will set $\mu_{X}=\mu_{Y}, \sigma_{X}^{s y s}=\sigma_{Y}$, and $\sigma_{X}^{i d}=0$.

To derive a closed-form expression for the price-dividend ratio in (A15), we use (16) to write the equilibrium state-price density as

$$
\pi_{t}=\sum_{n=0}^{\infty} a_{n, 1}^{\pi} \hat{\pi}_{1, t}^{1-\frac{n}{\gamma_{2}}} \hat{\pi}_{2, t}^{\frac{n}{\gamma_{2}}} 1_{\left\{\hat{\pi}_{1, t}>R\right\}}+\sum_{n=0}^{\infty} a_{n, 2}^{\pi} \hat{\pi}_{1, t}^{\frac{n}{\gamma_{1}}} \hat{\pi}_{2, t}^{1-\frac{n}{\gamma_{1}}} 1_{\left\{\frac{\hat{\pi}_{1, t}}{\hat{\pi}_{2, t}}<R\right\}} .
$$

Since the event $\left\{\begin{array}{l}\hat{\pi}_{1, t} \\ \hat{\pi}_{2, t}\end{array}=R\right\}$ is of measure zero, it follows from (A15) that

$$
v_{t, u}^{X}=\left(\pi_{t} X_{t}\right)^{-1} j_{t, u}
$$

where

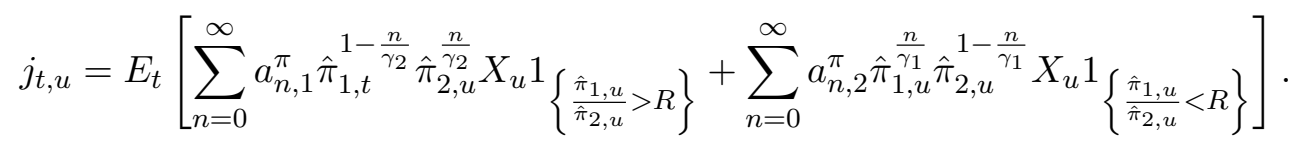

Since the two infinite series in the above expression stem from $\nu_{2, t}^{-\gamma_{2}}$ in (A11), and $\nu_{1, t}^{-\gamma_{1}}$ in (A13), which are complex analytic for $A \in \mathbb{C}$ such that $|A|<\bar{R}$, and $|A|>\bar{R}$, respectively, we can interchange the conditional expectation with the infinite sum to obtain

$$
j_{t, u}=\sum_{n=0}^{\infty} a_{n, 1}^{\pi} E_{t}\left[\hat{\pi}_{1, u}^{1-\frac{n}{\gamma_{2}}} \hat{\pi}_{2, u}^{\frac{n}{\gamma_{2}}} X_{u} 1_{\left\{\frac{\hat{\pi}_{1, u}}{\hat{\pi}_{2, u}}>R\right\}}\right]+\sum_{n=0}^{\infty} a_{n, 2}^{\pi} E_{t}\left[\hat{\pi}_{1, u}^{\frac{n}{\gamma_{1}} \hat{\pi}_{2, u}^{1-\frac{n}{\gamma_{1}}}} X_{u} 1_{\left\{\frac{\hat{\pi}_{1, u}}{\hat{\pi}_{2, u}}<R\right\}}\right] .
$$


We now rewrite the above expression as follows:

$$
j_{t, u}=\pi_{t} X_{t}\left(\sum_{n=0}^{\infty} \omega_{n, 1, t} \phi_{n, 1, t, u}^{X}+\sum_{n=0}^{\infty} \omega_{n, 2, t} \phi_{n, 2, t, u}^{X}\right),
$$

where $\omega_{n, 1, t}$ and $\omega_{n, 2, t}$ are given by

$$
\begin{aligned}
& \omega_{n, 1, t}=a_{n, 1}^{\pi} \frac{\hat{\pi}_{1, t}^{1-\frac{n}{\gamma_{2}}} \hat{\pi}_{2, t}^{\frac{n}{\gamma_{2}}}}{\pi_{t}}, n \in \mathbb{N}_{0}, \\
& \omega_{n, 2, t}=a_{n, 2}^{\pi} \frac{\hat{\pi}_{1, t}^{\frac{n}{\gamma_{1}}} \hat{\pi}_{2, t}^{1-\frac{n}{\gamma_{1}}}}{\pi_{t}}, n \in \mathbb{N}_{0},
\end{aligned}
$$

and $\phi_{n, 1, t, u}^{X}$ and $\phi_{n, 2, t, u}^{X}$ are given by

$$
\begin{aligned}
& \phi_{n, 1, t, u}^{X}=E_{t}\left[\frac{\hat{\pi}_{1, u}^{1-\frac{n}{\gamma_{2}}} \hat{\pi}_{2, u}^{\frac{n}{\gamma_{2}}}}{\hat{\pi}_{1, t}^{1-\frac{n}{\gamma_{2}}} \hat{\pi}_{2, t}^{\frac{n}{\gamma_{2}}}} \frac{X_{u}}{X_{t}} 1_{\left\{\frac{\hat{\pi}_{1, u}}{\hat{\pi}_{2, u}}>R\right\}}\right], n \in \mathbb{N}_{0}, \\
& \phi_{n, 2, t, u}^{X}=E_{t}\left[\frac{\hat{\pi}_{1, u}^{\frac{n}{\gamma_{1}}} \hat{\pi}_{2, u}^{1-\frac{n}{\gamma_{1}}}}{\hat{\pi}_{1, t}^{\frac{n}{\gamma_{1}}} \hat{\pi}_{2, t}^{1-\frac{n}{\gamma_{1}}}} \frac{X_{u}}{X_{t}} 1\left\{\frac{\hat{\pi}_{1, u}}{\hat{\pi}_{2, u}}<R\right\}\right], n \in \mathbb{N}_{0} .
\end{aligned}
$$

We now set $\mu_{X}=\mu_{Y}, \sigma_{X}^{\text {sys }}=\sigma_{Y}$, and $\sigma_{X}^{i d}=0$, and so Equation (25) follows from (A16) and (A17). We also note that (28) follows from (16), (A18), and (A19).

We now express the weights, $\omega_{n, 1, t}$ and $\omega_{n, 2, t}$, in terms of the consumption shares, $\nu_{1, t}$ and $\nu_{2, t}$. Equation (11) implies that for all $a \in \mathbb{R}$

$$
\pi_{t}=\hat{\pi}_{1, t}^{a} \nu_{1, t}^{-a \gamma_{1}} \hat{\pi}_{2, t}^{1-a} \nu_{2, t}^{-(1-a) \gamma_{2}}
$$

which implies that

$$
\hat{\pi}_{1, t}^{a} \hat{\pi}_{2, t}^{1-a}=\pi_{t} \nu_{1, t}^{a \gamma_{1}} \nu_{2, t}^{(1-a) \gamma_{2}}
$$

Therefore, we can rewrite the weights, $\omega_{n, 1, t}$ and $\omega_{n, 2, t}$, given in (A18) and (A19) as (26) and (27), respectively.

\section{A.5 Proof of Corollary 2: Price of dividend strip with identical risk aversion}

Again, rather than considering dividend strips where the dividend is the aggregate endowment, we shall derive results for a more general dividend process, $X$, where the evolution of $X$ is given by (A51). Then, to obtain the results in the proposition, we will set $\mu_{X}=\mu_{Y}, \sigma_{X}^{\text {sys }}=\sigma_{Y}$, and $\sigma_{X}^{i d}=0$. 
By setting $\gamma_{1}=\gamma_{1}=\gamma,(26)$ and (27) reduce to (31) and (32), respectively, and (29) and (30) reduce to

$$
\begin{aligned}
& \left.\phi_{n, 1, t, u}^{X}=E_{t}\left[\frac{\hat{\pi}_{1, u}^{1-\frac{n}{\gamma}} \hat{\pi}_{2, u}^{\frac{n}{\gamma}}}{\hat{\pi}_{1, t}^{1-\frac{n}{\gamma}} \hat{\pi}_{2, t}^{\frac{n}{\gamma}}} \frac{X_{u}}{X_{t}} 1_{\left\{\hat{\pi}_{1, u}\right.} \hat{\pi}_{2, u}>R\right\}\right], n \in \mathbb{N}_{0}, \\
& \left.\phi_{n, 2, t, u}^{X}=E_{t}\left[\frac{\hat{\pi}_{1, u}^{\frac{n}{\gamma}} \hat{\pi}_{2, u}^{1-\frac{n}{\gamma}}}{\hat{\pi}_{1, t}^{\frac{n}{\gamma}} \hat{\pi}_{2, t}^{1-\frac{n}{\gamma}}} \frac{X_{u}}{X_{t}} 1_{\left\{\hat{\pi}_{1, u}\right.} \hat{\pi}_{2, u}<R\right\}\right], n \in \mathbb{N}_{0} .
\end{aligned}
$$

When $\gamma \in \mathbb{N},(25)$ reduces to

$$
\begin{aligned}
v_{t, u}^{X} & =\sum_{n=0}^{\gamma} \omega_{n, 1, t} \phi_{n, 1, t, u}^{X}+\sum_{n=0}^{\gamma} \omega_{n, 2, t} \phi_{n, 2, t, u}^{X} \\
& =\sum_{n=0}^{\gamma} \omega_{n, t}\left(\phi_{n, 1, t, u}^{X}+\phi_{\gamma-n, 2, t, u}^{X}\right)
\end{aligned}
$$

where $\omega_{n, t}$ is given in (34). It follows from (A20) and (A21) that

$$
\phi_{n, 1, t}^{X}+\phi_{\gamma-n, 2, t}^{X}=E_{t}\left[\frac{\hat{\pi}_{1, u}^{1-\frac{n}{\gamma}} \hat{\pi}_{2, u}^{\frac{n}{\gamma}}}{\hat{\pi}_{1, t}^{1-\frac{n}{\gamma}} \hat{\pi}_{2, t}^{\frac{n}{\gamma}}} \frac{X_{u}}{X_{t}}\right], n \in \mathbb{N}_{0} \text { and } n \leq \gamma
$$

The $n$ 'th weight in the sum is given by the expression in (34); observe that the weights sum to one, because

$$
\sum_{n=0}^{\gamma}\left(\begin{array}{l}
\gamma \\
n
\end{array}\right)\left(\nu_{1, t}^{1-\frac{n}{\gamma}} \nu_{2, t}^{\frac{n}{\gamma}}\right)^{\gamma}=\left(\nu_{1, t}+\nu_{2, t}\right)^{\gamma}=1
$$

\section{A.6 Proof of Proposition 4: Dynamics of the consumption-sharing rule}

We can see the state variables of the economy by writing the consumption sharing rule as

$$
e^{\Delta_{t}} \nu_{1, t}^{-\gamma_{1}}=\nu_{2, t}^{-\gamma_{2}},
$$

where

$$
\Delta_{t}=\ln \frac{\hat{\pi}_{1, t}}{\hat{\pi}_{2, t}}=q_{t}+d_{\omega} \omega_{t}
$$

and

$$
\begin{aligned}
q_{t} & =\ln \frac{\lambda_{1,0}}{\lambda_{2,0}}+\mu_{q} t+\sigma_{q} Z_{t} \\
\mu_{q} & =\left(\beta_{2}-\beta_{1}\right)+\frac{1}{2}\left(\sigma_{\xi, 2}^{2}-\sigma_{\xi, 1}^{2}\right)+\left(\frac{1}{\psi_{2}}-\frac{1}{\psi_{1}}\right)\left(\mu_{Y}-\frac{1}{2} \sigma_{Y}^{2}\right),
\end{aligned}
$$




$$
\begin{aligned}
& \sigma_{q}=\sigma_{\xi, 1}-\sigma_{\xi, 2}+\left(\frac{1}{\psi_{2}}-\frac{1}{\psi_{1}}\right) \sigma_{Y} \\
& d_{\omega}=\left(\gamma_{2}-\frac{1}{\psi_{2}}\right)-\left(\gamma_{1}-\frac{1}{\psi_{1}}\right) .
\end{aligned}
$$

Hence, the state variables of the economy are $q$ and $\omega$.

We observe from (15) that the evolution of the ratio $\frac{\hat{\pi}_{1, t}}{\hat{\pi}_{2, t}}$ will have a deterministic component and a stochastic component, where the stochastic component depends on the stochastic behavior of aggregate endowment and the differences in beliefs.

From Ito's Lemma

$$
d \nu_{1, t}=\frac{\partial \nu_{1, t}}{\partial \Delta_{t}} d \Delta_{t}+\frac{1}{2} \frac{\partial^{2} \nu_{1, t}}{\partial \Delta_{t}^{2}}\left(d \Delta_{t}\right)^{2}
$$

Differentiating (A22) implicitly with respect $\Delta_{t}$ and solving for $\frac{\partial \nu_{1, t}}{\partial \Delta_{t}}$ gives

$$
\frac{\partial \nu_{1, t}}{\partial \Delta_{t}}=\mathbf{R}_{t} \frac{\nu_{1, t} \nu_{2, t}}{\gamma_{1} \gamma_{2}}
$$

Differentiating (A23) with respect to $\Delta_{t}$ gives

$$
\begin{aligned}
\frac{\partial^{2} \nu_{1, t}}{\partial \Delta_{t}^{2}} & =\frac{\partial}{\partial \Delta_{t}}\left[\mathbf{R}_{t} \frac{\nu_{1, t} \nu_{2, t}}{\gamma_{1} \gamma_{2}}\right] \\
& =\frac{1}{\gamma_{1} \gamma_{2}} \frac{\partial}{\partial \Delta_{t}}\left[\mathbf{R}_{t} \nu_{1, t} \nu_{2, t}\right] \\
& =\frac{1}{\gamma_{1} \gamma_{2}}\left[\mathbf{R}_{t} \frac{\partial}{\partial \Delta_{t}}\left[\nu_{1, t} \nu_{2, t}\right]+\nu_{1, t} \nu_{2, t} \frac{\partial \mathbf{R}_{t}}{\partial \Delta_{t}}\right] .
\end{aligned}
$$

We simplify the above expression by observing that

$$
\frac{\partial \mathbf{R}_{t}}{\partial \Delta_{t}}=-\mathbf{R}_{t}^{2}\left(\frac{1}{\gamma_{1}}-\frac{1}{\gamma_{2}}\right) \frac{\partial \nu_{1, t}}{\partial \Delta_{t}} .
$$

and using (A23) to obtain

$$
\frac{\partial^{2} \nu_{1, t}}{\partial \Delta_{t}^{2}}=\nu_{1, t} \nu_{2, t}\left(\frac{\mathbf{R}_{t}}{\gamma_{1} \gamma_{2}}\right)^{2}\left[\left(\nu_{2, t}-\nu_{1, t}\right)-\nu_{1, t} \nu_{2, t} \mathbf{R}_{t}\left(\frac{1}{\gamma_{1}}-\frac{1}{\gamma_{2}}\right)\right] .
$$

Hence,

$$
\begin{aligned}
d \nu_{1, t} & =\frac{\partial \nu_{1, t}}{\partial \Delta_{t}} d \Delta_{t}+\frac{1}{2} \frac{\partial^{2} \nu_{1, t}}{\partial \Delta_{t}^{2}}\left(d \Delta_{t}\right)^{2} \\
& =\nu_{1, t} \nu_{2, t} \frac{\mathbf{R}_{t}}{\gamma_{1} \gamma_{2}}\left\{\mu_{q} d t+\sigma_{q} d Z_{t}+d_{\omega}\left[\lambda_{x}\left(\bar{\omega}-\omega_{t}\right)\right] d t+d_{\omega} \sigma_{Y} d Z_{t}\right. \\
& \left.+\frac{1}{2} \frac{\mathbf{R}_{t}}{\gamma_{1} \gamma_{2}}\left[\nu_{2, t}-\nu_{1, t}-\nu_{1, t} \nu_{2, t} \mathbf{R}_{t}\left(\frac{1}{\gamma_{1}}-\frac{1}{\gamma_{2}}\right)\right]\left(\sigma_{q}+d_{\omega} \sigma_{Y}\right)^{2} d t\right\} .
\end{aligned}
$$


Now observe that

$$
\begin{aligned}
\sigma_{q}+d_{\omega} \sigma_{Y} & =\sigma_{\xi, 1}-\sigma_{\xi, 2}+\left(\frac{1}{\psi_{2}}-\frac{1}{\psi_{1}}\right) \sigma_{Y}+\left(\gamma_{2}-\frac{1}{\psi_{2}}\right)-\left(\gamma_{1}-\frac{1}{\psi_{1}}\right) \sigma_{Y} \\
& =\sigma_{\xi, 1}-\sigma_{\xi, 2}+\left(\gamma_{2}-\gamma_{1}\right) \sigma_{Y}
\end{aligned}
$$

Therefore

$$
\begin{aligned}
d \nu_{1, t} & =\nu_{1, t} \nu_{2, t} \frac{\mathbf{R}_{t}}{\gamma_{1} \gamma_{2}}\left\{\mu_{q}+d_{\omega}\left[\lambda_{x}\left(\bar{\omega}-\omega_{t}\right)\right]\right. \\
& \left.+\frac{1}{2} \frac{\mathbf{R}_{t}}{\gamma_{1} \gamma_{2}}\left[\nu_{2, t}-\nu_{1, t}-\nu_{1, t} \nu_{2, t} \mathbf{R}_{t}\left(\frac{1}{\gamma_{1}}-\frac{1}{\gamma_{2}}\right)\right]\left[\sigma_{\xi, 1}-\sigma_{\xi, 2}+\left(\gamma_{2}-\gamma_{1}\right) \sigma_{Y}\right]^{2}\right\} d t \\
& +\nu_{1, t} \nu_{2, t} \frac{\mathbf{R}_{t}}{\gamma_{1} \gamma_{2}}\left[\sigma_{\xi, 1}-\sigma_{\xi, 2}+\left(\gamma_{2}-\gamma_{1}\right) \sigma_{Y}\right] d Z_{t} .
\end{aligned}
$$

Now observe that

$$
\begin{aligned}
& \mu_{q}+d_{\omega}\left[\lambda_{x}\left(\bar{\omega}-\omega_{t}\right)\right] \\
& =\left(\beta_{2}-\beta_{1}\right)+\frac{1}{2}\left(\sigma_{\xi, 2}^{2}-\sigma_{\xi, 1}^{2}\right)+\left(\frac{1}{\psi_{2}}-\frac{1}{\psi_{1}}\right)\left(\mu_{Y}-\frac{1}{2} \sigma_{Y}^{2}\right) \\
& {\left[\left(\gamma_{2}-\frac{1}{\psi_{2}}\right)-\left(\gamma_{1}-\frac{1}{\psi_{1}}\right)\right]\left(\mu_{Y}-\frac{1}{2} \sigma_{Y}^{2}\right)-\left[\left(\gamma_{2}-\frac{1}{\psi_{2}}\right)-\left(\gamma_{1}-\frac{1}{\psi_{1}}\right)\right] \lambda_{x} \omega_{t}} \\
& =\beta_{2}-\beta_{1}+\frac{1}{2}\left(\sigma_{\xi, 2}^{2}-\sigma_{\xi, 1}^{2}\right)+\left(\gamma_{2}-\gamma_{1}\right)\left(\mu_{Y}-\frac{1}{2} \sigma_{Y}^{2}\right)-\left[\left(\gamma_{2}-\frac{1}{\psi_{2}}\right)-\left(\gamma_{1}-\frac{1}{\psi_{1}}\right)\right] \lambda_{x} \omega_{t}
\end{aligned}
$$

and

$$
\begin{aligned}
\frac{\mathbf{R}_{t}}{\gamma_{1} \gamma_{2}}\left[\nu_{2, t}-\nu_{1, t}-\nu_{1, t} \nu_{2, t} \mathbf{R}_{t}\left(\frac{1}{\gamma_{1}}-\frac{1}{\gamma_{2}}\right)\right] & =\left(\frac{\mathbf{R}_{t} \nu_{2, t}}{\gamma_{2}}\right) \frac{1}{\gamma_{1}}-\left(\frac{\mathbf{R}_{t} \nu_{1, t}}{\gamma_{1}}\right) \frac{1}{\gamma_{2}}-\frac{\mathbf{R}_{t} \nu_{1, t}}{\gamma_{1}} \frac{\mathbf{R}_{t} \nu_{2, t}}{\gamma_{2}}\left(\frac{1}{\gamma_{1}}-\frac{1}{\gamma_{2}}\right) \\
& =\frac{w_{2, t}}{\gamma_{1}}-\frac{w_{1, t}}{\gamma_{2}}-w_{1, t} w_{2, t}\left(\frac{1}{\gamma_{1}}-\frac{1}{\gamma_{2}}\right)=\frac{w_{2, t}^{2}}{\gamma_{1}}-\frac{w_{1, t}^{2}}{\gamma_{2}}=\frac{\gamma_{2} w_{2, t}^{2}-\gamma_{1} w_{1, t}^{2}}{\gamma_{1} \gamma_{2}}
\end{aligned}
$$

Hence,

$$
\begin{aligned}
d \nu_{1, t} & =\nu_{1, t} \nu_{2, t} \frac{\mathbf{R}_{t}}{\gamma_{1} \gamma_{2}} \times \\
& \left\{\left(\beta_{2}-\beta_{1}\right)+\frac{1}{2}\left(\sigma_{\xi, 2}^{2}-\sigma_{\xi, 1}^{2}\right)+\left(\gamma_{2}-\gamma_{1}\right)\left(\mu_{Y}-\frac{1}{2} \sigma_{Y}^{2}\right)-\left[\left(\gamma_{2}-\frac{1}{\psi_{2}}\right)-\left(\gamma_{1}-\frac{1}{\psi_{1}}\right)\right] \lambda_{x} \omega_{t}\right. \\
& \left.+\frac{1}{2} \frac{\gamma_{2} w_{2, t}^{2}-\gamma_{1} w_{1, t}^{2}}{\gamma_{1} \gamma_{2}}\left[\left(\sigma_{\xi, 1}-\sigma_{\xi, 2}\right)+\left(\gamma_{2}-\gamma_{1}\right) \sigma_{Y}\right]^{2}\right\} d t \\
& +\nu_{1, t} \nu_{2, t} \frac{\mathbf{R}_{t}}{\gamma_{1} \gamma_{2}}\left[\left(\sigma_{\xi, 1}-\sigma_{\xi, 2}\right)+\left(\gamma_{2}-\gamma_{1}\right) \sigma_{Y}\right] d Z_{t} .
\end{aligned}
$$




$$
\begin{aligned}
& =\nu_{1, t} \nu_{2, t} \frac{\mathbf{R}_{t}}{\gamma_{1} \gamma_{2}}\left\{\left(\beta_{2}-\beta_{1}\right)+\frac{1}{2}\left(\sigma_{\xi, 2}^{2}-\sigma_{\xi, 1}^{2}\right)\right. \\
& +\left(\frac{1}{\psi_{2}}-\frac{1}{\psi_{1}}\right)\left(\mu_{Y}-\frac{1}{2} \sigma_{Y}^{2}\right)+\left[\left(\gamma_{2}-\frac{1}{\psi_{2}}\right)-\left(\gamma_{1}-\frac{1}{\psi_{1}}\right)\right] \lambda_{x}\left(\bar{\omega}-\omega_{t}\right) \\
& \left.+\frac{1}{2} \frac{\gamma_{2} w_{2, t}^{2}-\gamma_{1} w_{1, t}^{2}}{\gamma_{1} \gamma_{2}}\left[\left(\sigma_{\xi, 1}-\sigma_{\xi, 2}\right)+\left(\gamma_{2}-\gamma_{1}\right) \sigma_{Y}\right]^{2}\right\} d t \\
& +\nu_{1, t} \nu_{2, t} \frac{\mathbf{R}_{t}}{\gamma_{1} \gamma_{2}}\left[\left(\sigma_{\xi, 1}-\sigma_{\xi, 2}\right)+\left(\gamma_{2}-\gamma_{1}\right) \sigma_{Y}\right] d Z_{t} \\
& =\nu_{1, t} \nu_{2, t} \frac{\mathbf{R}_{t}}{\gamma_{1} \gamma_{2}}\left\{\beta_{2}-\beta_{1}+\left(\frac{\mu_{Y, 1}+\mu_{Y, 2}}{2}-\mu_{Y}\right)\left(\frac{\mu_{Y, 2}-\mu_{Y, 1}}{\sigma_{Y}}\right)\right. \\
& +\left(\frac{1}{\psi_{2}}-\frac{1}{\psi_{1}}\right)\left(\mu_{Y}-\frac{1}{2} \sigma_{Y}^{2}\right)+\left[\left(\gamma_{2}-\frac{1}{\psi_{2}}\right)-\left(\gamma_{1}-\frac{1}{\psi_{1}}\right)\right] \lambda_{x}\left(\bar{\omega}-\omega_{t}\right) \\
& \left.+\frac{1}{2} \frac{\gamma_{2} w_{2, t}^{2}-\gamma_{1} w_{1, t}^{2}}{\gamma_{1} \gamma_{2}}\left[\left(\frac{\mu_{Y, 1}-\mu_{Y, 2}}{\sigma_{Y}}\right)^{2}+2\left(\mu_{Y, 1}-\mu_{Y, 2}\right)\left(\gamma_{2}-\gamma_{1}\right)+\left(\gamma_{2}-\gamma_{1}\right)^{2} \sigma_{Y}^{2}\right]\right\} d t \\
& +\nu_{1, t} \nu_{2, t} \frac{\mathbf{R}_{t}}{\gamma_{1} \gamma_{2}}\left[\frac{\mu_{Y, 1}-\mu_{Y, 2}}{\sigma_{Y}}+\left(\gamma_{2}-\gamma_{1}\right) \sigma_{Y}\right] d Z_{t} .
\end{aligned}
$$

\section{A.7 Proof of Proposition 5: Riskfree rate}

We know that the equilibrium state price density, $\pi$, is given by

$$
\pi_{t}=\hat{\pi}_{1, t} \nu_{1, t}^{-\gamma_{1}}=\hat{\pi}_{2, t} \nu_{2, t}^{-\gamma_{2}}
$$

Hence,

$$
\ln \pi_{t}=\ln \hat{\pi}_{1, t}-\gamma_{1} \ln \nu_{1, t}
$$

In the homogeneous agent economy where all agents are of Type $k$, we derive the risk-free rate, $\hat{r}_{k, t}$ and the market price of risk, $\hat{\theta}_{k, t}$. We starting from the well-known result that

$$
\frac{d \hat{\pi}_{k, t}}{\hat{\pi}_{k, t}}=-\hat{r}_{k, t} d t-\hat{\theta}_{k, t} d Z_{t}
$$

Given our assumptions for the aggregate endowment, we can apply Ito's Lemma to the last expression in (12)

$$
\begin{aligned}
d \ln \hat{\pi}_{k, t} & =-\frac{1}{2} \sigma_{\xi, k}^{2} d t+\sigma_{\xi, k} d Z_{t}-\beta_{k} d t-\left(\gamma_{k}-\frac{1}{\psi_{k}}\right) d \omega_{t}+\frac{1}{\psi_{k}} d y_{t} \\
& =-\frac{1}{2} \sigma_{\xi, k}^{2} d t-\beta_{k} d t-\left(\gamma_{k}-\frac{1}{\psi_{k}}\right)\left[\lambda_{x}(\bar{\omega}-\omega) d t+\sigma_{Y} d Z_{t}\right]+\frac{1}{\psi_{k}}\left[\left(\mu_{Y}-\frac{1}{2} \sigma_{Y}^{2}\right) d t+\sigma_{Y} d Z_{t}\right] .
\end{aligned}
$$


Therefore

$$
\hat{\theta}_{k}=-\sigma_{\xi, k}+\left(\gamma_{k}-\frac{1}{\psi_{k}}\right) \sigma_{Y}+\frac{1}{\psi_{k}} \sigma_{Y}=\gamma_{k} \sigma_{Y}+\frac{\mu_{Y}-\mu_{Y, k}}{\sigma_{Y}}
$$

and

$$
r_{k, t}+\frac{1}{2} \hat{\theta}_{k, t}^{2}=r_{k, t}+\frac{1}{2}\left(\gamma_{k} \sigma_{Y}-\sigma_{\xi, k}\right)=\frac{1}{2} \sigma_{\xi, k}^{2}+\beta_{k}+\left(\gamma_{k}-\frac{1}{\psi_{k}}\right) \lambda_{x}\left(\bar{\omega}-\omega_{t}\right)+\frac{1}{\psi_{k}}\left(\mu_{Y}-\frac{1}{2} \sigma_{Y}^{2}\right) .
$$

Hence,

$$
\begin{aligned}
\hat{r}_{k, t}+\frac{1}{2}\left(\sigma_{\xi, k}^{2}-2 \gamma_{k} \sigma_{Y} \sigma_{\xi, k}+\gamma_{k}^{2} \sigma_{\xi, k}^{2}\right) & =\frac{1}{2} \sigma_{\xi, k}^{2}+\beta_{k}+\left(\gamma_{k}-\frac{1}{\psi_{k}}\right) \lambda_{x}\left(\bar{\omega}-\omega_{t}\right)+\frac{1}{\psi_{k}}\left(\mu_{Y}-\frac{1}{2} \sigma_{Y}^{2}\right) \\
\hat{r}_{k, t}-\gamma_{k}\left(\mu_{Y, k}-\mu_{Y}\right)+\frac{1}{2} \gamma_{k}^{2} \sigma_{Y}^{2} & =\beta_{k}+\left(\gamma_{k}-\frac{1}{\psi_{k}}\right) \lambda_{x}\left(\bar{\omega}-\omega_{t}\right)+\frac{1}{\psi_{k}}\left(\mu_{Y}-\frac{1}{2} \sigma_{Y}^{2}\right),
\end{aligned}
$$

and so

$$
\hat{r}_{k, t}=\beta_{k}+\frac{1}{\psi_{k}} \mu_{Y, k}+\lambda_{x}\left(\bar{\omega}_{k}-\omega_{t}\right)\left(\gamma_{k}-\frac{1}{\psi_{k}}\right)-\frac{1}{2}\left[\gamma_{k}\left(1+\gamma_{k}\right)-\left(\gamma_{k}-\frac{1}{\psi_{k}}\right)\right] \sigma_{Y}^{2},
$$

where

$$
\bar{\omega}_{k}=\frac{\mu_{Y, k}-\frac{1}{2} \sigma_{Y}^{2}}{\lambda_{x}}
$$

Applying Ito's Lemma to $\ln \pi_{t}$ gives

$$
\begin{aligned}
d \ln \pi_{t} & =d \ln \hat{\pi}_{1, t}-\gamma_{1} d \ln \nu_{1, t}=-\left(\hat{r}_{1, t}+\frac{1}{2} \hat{\theta}_{1, t}^{2}\right) d t-\hat{\theta}_{1, t} d Z_{t}-\gamma_{1}\left[\left(\mu_{\nu_{1}, t}-\frac{1}{2} \sigma_{\nu_{1}, t}^{2}\right) d t+\sigma_{\nu_{1}, t} d Z_{t}\right] \\
& =-\left[\hat{r}_{1, t}+\frac{1}{2} \hat{\theta}_{1, t}^{2}+\gamma_{1}\left(\mu_{\nu_{1}, t}-\frac{1}{2} \sigma_{\nu_{1}, t}^{2}\right)\right] d t-\left(\hat{\theta}_{1, t}+\gamma_{1} \sigma_{\nu_{1}, t}\right) d Z_{t} .
\end{aligned}
$$

Therefore

$$
\begin{aligned}
\theta_{t} & =\hat{\theta}_{1, t}+\gamma_{1} \sigma_{\nu_{1}, t} \\
= & \frac{\mu_{Y}-\mu_{Y, 1}}{\sigma_{Y}}+\gamma_{1} \sigma_{Y}+\frac{\nu_{1, t} \mathbf{R}_{t}}{\gamma_{2}}\left[\frac{\mu_{Y, 1}-\mu_{Y, 2}}{\sigma_{Y}}+\left(\gamma_{2}-\gamma_{1}\right) \sigma_{Y}\right] \\
= & \frac{\mu_{Y}-\left[\left(1-\frac{\nu_{2, t} \mathbf{R}_{t}}{\gamma_{2}}\right) \mu_{Y, 1}+\frac{\nu_{2, t} \mathbf{R}_{t}}{\gamma_{2}} \mu_{Y, 2}\right]}{\sigma_{Y}}+\left[\gamma_{1}\left(1-\frac{\nu_{2, t} \mathbf{R}_{t}}{\gamma_{2}}\right)+\gamma_{2} \frac{\nu_{2, t} \mathbf{R}_{t}}{\gamma_{2}}\right] \sigma_{Y} \\
= & \frac{\mu_{Y}-\left(\frac{\nu_{1, t} \mathbf{R}_{t}}{\gamma_{1}} \mu_{Y, 1}+\frac{\nu_{2, t} \mathbf{R}_{t}}{\gamma_{2}} \mu_{Y, 2}\right)}{\sigma_{Y}}+\left(\gamma_{1} \frac{\nu_{1, t} \mathbf{R}_{t}}{\gamma_{1}}+\gamma_{2} \frac{\nu_{2, t} \mathbf{R}_{t}}{\gamma_{2}}\right) \sigma_{Y} \\
= & \frac{\mu_{Y}-\left(\frac{\nu_{1, t} \mathbf{R}_{t}}{\gamma_{1}} \mu_{Y, 1}+\frac{\nu_{2, t} \mathbf{R}_{t}}{\gamma_{2}} \mu_{Y, 2}\right)}{\sigma_{Y}}+\mathbf{R}_{t} \sigma_{Y}
\end{aligned}
$$

where we have used the fact that

$$
\frac{\nu_{1, t} \mathbf{R}_{t}}{\gamma_{1}}=1-\frac{\nu_{2, t} \mathbf{R}_{t}}{\gamma_{2}}
$$


Therefore,

$$
\theta_{t}=\frac{\mu_{Y}-\boldsymbol{\mu}_{Y, t}}{\sigma_{Y}}+\mathbf{R}_{t} \sigma_{Y}
$$

where

$$
\boldsymbol{\mu}_{Y, t}=\sum_{k=1}^{2} w_{k, t} \mu_{Y, k}
$$

and

$$
w_{k, t}=\frac{\nu_{k, t}}{\gamma_{k}} \mathbf{R}_{t}, k \in\{1,2\}
$$

Also,

$$
r_{t}+\frac{1}{2} \theta_{t}^{2}=\hat{r}_{1, t}+\gamma_{1}\left(\mu_{\nu_{1}, t}-\frac{1}{2} \sigma_{\nu_{1}, t}^{2}\right)+\frac{1}{2} \hat{\theta}_{1, t}^{2} .
$$

Therefore,

$$
\begin{aligned}
& r_{t}=\hat{r}_{1, t}+\gamma_{1}\left(\mu_{\nu_{1}, t}-\frac{1}{2} \sigma_{\nu_{1}, t}^{2}\right)+\frac{1}{2} \hat{\theta}_{1, t}^{2}-\frac{1}{2}\left(\hat{\theta}_{1, t}^{2}+2 \gamma_{1} \hat{\theta}_{1, t}^{2} \sigma_{\nu_{1}, t}+\gamma_{1}^{2} \sigma_{\nu_{1}, t}^{2}\right) \\
& r_{t}=r_{1, t}+\gamma_{1}\left(\mu_{\nu_{1}, t}-\frac{1}{2} \sigma_{\nu_{1}, t}\right)-\gamma_{1} \hat{\theta}_{1} \sigma_{\nu_{1}, t}-\frac{1}{2} \gamma_{1}^{2} \sigma_{\nu_{1}, t}^{2}
\end{aligned}
$$

Substituting (A24) and $\mu_{\nu_{1}, t}, \sigma_{\nu_{1}, t}$ from Proposition 4 into the above expression and simplifying gives

$$
\begin{aligned}
r_{t} & =\boldsymbol{\beta}_{t}+\mathbf{R}_{t} \boldsymbol{\mu}_{Y, t}-\frac{1}{2} \mathbf{R}_{t} \mathbf{P}_{t} \sigma_{Y}^{2}-\lambda_{x} \omega_{t}\left[\mathbf{R}_{t}-\left(\frac{w_{1, t}}{\psi_{1}}+\frac{w_{2, t}}{\psi_{2}}\right)\right] \\
& +\frac{1}{2} w_{1, t} w_{2, t}\left(1-\frac{\mathbf{R}_{t}}{\gamma_{1} \gamma_{2}}\right)\left(\frac{\mu_{Y, 1}-\mu_{Y, 2}}{\sigma_{Y}}\right)^{2}-w_{1, t} w_{2, t} \mathbf{R}_{t}\left(\frac{1}{\gamma_{1}}-\frac{1}{\gamma_{2}}\right)\left(\mu_{Y, 1}-\mu_{Y, 2}\right) .
\end{aligned}
$$

We can rewrite the above expression as (48).

\section{A.8 Proof of Corollary 3: Riskfree rate with correct beliefs or with identical risk aversions and habits}

Equation (50) follows from (48) after setting $\mu_{Y, 1}=\mu_{Y, 2}=\mu_{Y}$, and simplifying. Equation (51) follows from (48) after setting $\gamma_{1}=\gamma_{2}=\gamma$ and $\psi_{1}=\psi_{2}=\psi$, and simplifying.

\section{A.9 Proof of Proposition 6: Market price of risk}

We have (54) from (A25). Given that the $\xi_{k, t}$ is the exponential martingale defining the change of probability measure from $\mathbb{P}$ to $\mathbb{P}^{k}$, it follows from Girsanov's Theorem that Agent $k$ 's perception of the market price of risk is

$$
\theta_{k, t}=\theta_{t}+\sigma_{\xi, k}
$$


Hence,

$$
\theta_{k, t}=\theta_{t}+\sigma_{\xi, k}=\mathbf{R}_{t} \sigma_{Y}+\frac{\mu_{Y}-\boldsymbol{\mu}_{Y, t}}{\sigma_{Y}}+\frac{\mu_{Y, k}-\mu_{Y}}{\sigma_{Y}}=\mathbf{R}_{t} \sigma_{Y}+\frac{\mu_{Y, k}-\boldsymbol{\mu}_{Y, t}}{\sigma_{Y}}
$$

Thus, we obtain (55).

\section{A.10 Proof of Corollary 4: Market price of risk with correct beliefs or with identical risk aversions}

Equation (56) follows from (55) after setting $\mu_{Y, 1}=\mu_{Y, 2}=\mu_{Y}$, and simplifying. Equations (57) follow from (55) after setting $\gamma_{1}=\gamma_{2}=\gamma$, and simplifying.

\section{A.11 Proof of Proposition 7}

Equation (13) can be rewritten as

$$
\nu_{2, t}^{\gamma_{2}} e^{q_{t}+d_{\omega} \omega_{t}}=\nu_{1, t}^{\gamma_{1}}
$$

and so

$$
\nu_{2, t}^{\eta} e^{\frac{q_{t}+d_{\omega} \omega_{t}}{\gamma_{1}}}=\nu_{1, t}
$$

Now, recall the standard results that

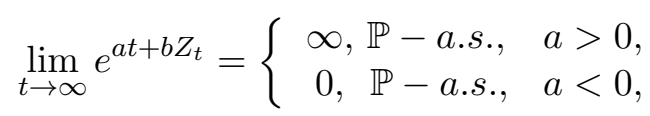

and

$$
\begin{aligned}
& \lim \sup _{t \rightarrow \infty} e^{b Z_{t}}=\infty \\
& \lim \inf _{t \rightarrow \infty} e^{b Z_{t}}=0 .
\end{aligned}
$$

From the above results it follows that to ensure that $\lim _{t \rightarrow \infty} e^{a t+b Z_{t}}$ is strictly between zero and infinity, we need to have both $a$ and $b$ equal to zero. It then follows from the expression in (A26) that both agents will survive $\mathbb{P}$-a.s., that is, the economy will be stationary almost surely under $\mathbb{P}$, if and only if $\mu_{q}=0$ and $\sigma_{q}=0$. We can also see that $\mu_{q}>0$ then Agent 2 survives, but Agent 1 does not. We can therefore define survival indices as in (58).

\section{A.12 Proof of Proposition 8}

To prove Proposition 8, we first prove the following three lemmas. 
Lemma A1 (Partial differential equation for price of a dividend strip) Given the aggregate endowment process specified in Equation (35) and the beliefs process in (36), the time-t price of the dividend strip, which pays the cash flow $Y_{u}$ at date $u>t$, is given by $V_{t, u}^{Y}=v_{t, u}^{Y} Y_{t}$, where

$$
\begin{aligned}
0 & =\frac{\partial v_{t, u}^{Y}}{\partial t}+\frac{\partial v_{t, u}^{Y}}{\partial \omega_{t}} \lambda_{x}\left(\bar{\omega}-\omega_{t}\right)+\nu_{1, t} \mu_{\nu_{1}, t} \frac{\partial v_{t, u}^{Y}}{\partial \nu_{1, t}} \\
& +\frac{1}{2} \sigma_{Y}^{2} \frac{\partial^{2} v_{t, u}^{Y}}{\partial \omega_{t}^{2}}+\sigma_{Y} \nu_{1, t} \sigma_{\nu_{1}, t} \frac{\partial^{2} v_{t, u}^{Y}}{\partial \omega_{t} \partial \nu_{1, t}}+\frac{1}{2} \nu_{1, t}^{2} \sigma_{\nu_{1}, t}^{2} \frac{\partial^{2} v_{t, u}^{Y}}{\partial \nu_{1, t}^{2}} \\
& +\left(\mu_{Y}-\theta_{t} \sigma_{Y}\right) v_{t, u}^{Y}+\left(\sigma_{Y}-\theta_{t}\right)\left(\sigma_{Y} \frac{\partial v_{t, u}^{Y}}{\partial \omega_{t}}+\nu_{1, t} \sigma_{\nu_{1}, t} \frac{\partial v_{t, u}^{Y}}{\partial \nu_{1, t}}\right)-r_{t} v_{t, u}^{Y}
\end{aligned}
$$

and

$$
\begin{aligned}
\left.v_{t, u}^{Y}\right|_{u=t} & =1 \\
\lim _{\omega_{t} \rightarrow \infty} v_{t, u}^{Y} & =\infty, \lim _{\omega_{t} \rightarrow-\infty} v_{t, u}^{Y}=0, \text { if } \gamma_{k}>\frac{1}{\psi_{k}}, k \in\{1,2\} \\
\left.v_{t, u}^{Y}\right|_{\nu_{k, t}=1} & =e^{-\left(\hat{r}_{k}+\frac{1}{\psi_{k}} \sigma_{Y}^{2}-\mu_{Y, k}\right)(u-t)} e^{-\left(\gamma_{k}-\frac{1}{\psi_{k}}\right)\left[\left(1-e^{-\lambda_{x}(u-t)}\right)\left(\hat{\omega}_{k}^{Y}-\omega_{t}\right)-\frac{1}{2}\left(\gamma_{k}-\frac{1}{\psi_{k}}\right) \frac{1-e^{-2 \lambda_{x}(u-t)}}{2 \lambda_{x}} \sigma_{Y}^{2}\right]},
\end{aligned}
$$

where

$$
\begin{aligned}
\hat{\omega}_{k}^{Y} & =\frac{\mu_{Y, k}+\left(1-\frac{1}{\psi_{k}}\right) \sigma_{Y}^{2}-\frac{1}{2} \sigma_{Y}^{2}}{\lambda_{x}} \\
\hat{r}_{k} & =\beta_{k}+\frac{1}{\psi_{k}} \mu_{Y, k}-\frac{1}{2} \frac{1}{\psi_{k}}\left(1+\frac{1}{\psi_{k}}\right) \sigma_{Y}^{2} .
\end{aligned}
$$

\section{Proof of Lemma A1}

The fundamental asset pricing equation (see Cochrane (2001)) states that

$$
E_{t}\left[d V_{t, u}^{Y}-r V_{t, u}^{Y} d t\right]=-E_{t}\left[\frac{d \pi_{t}}{\pi_{t}} d V_{t, u}^{Y}\right]
$$

Since $V_{t, u}^{Y}=Y_{t} v_{t, u}^{Y}$, where $v_{t, u}^{Y}$ is a function of time and the state variables $\omega_{t}$ and $\nu_{1, t}$, i.e. $v_{t, u}^{Y}=v_{t, u}^{Y}\left(t, \omega_{t}, \nu_{1, t}\right)$, it follows from Ito's Lemma that

$$
\begin{aligned}
\frac{d V_{t, u}^{Y}}{V_{t, u}^{Y}} & =\frac{d v_{t, u}^{Y}}{v_{t, u}^{Y}}+\frac{d Y_{t}}{Y_{t}}+\frac{d v_{t, u}^{Y}}{v_{t, u}^{Y}} \frac{d Y_{t}}{Y_{t}} \\
& =\frac{1}{v_{t, u}^{Y}}\left[\frac{\partial v_{t, u}^{Y}}{\partial t}+\frac{\partial v_{t, u}^{Y}}{\partial \omega_{t}} d \omega_{t}+\frac{\partial v_{t, u}^{Y}}{\partial \nu_{1, t}} d \nu_{1, t}+\frac{1}{2} \frac{\partial^{2} v_{t, u}^{Y}}{\partial \omega_{t}^{2}}\left(d \omega_{t}\right)^{2}+\frac{\partial^{2} v_{t, u}^{Y}}{\partial \omega_{t} \partial \nu_{1, t}} d \omega_{t} d \nu_{1, t}+\frac{1}{2} \frac{\partial^{2} v_{t, u}^{Y}}{\partial \omega_{t}^{2}}\left(d \nu_{1, t}\right)^{2}\right]
\end{aligned}
$$




$$
+\mu_{Y} d t+\sigma_{Y} d Z_{t}+\frac{1}{v_{t, u}^{Y}}\left(\frac{\partial v_{t, u}^{Y}}{\partial \omega_{t}} d \omega_{t}+\frac{\partial v_{t, u}^{Y}}{\partial \nu_{1, t}} d \nu_{1, t}\right) \frac{d Y_{t}}{Y_{t}}
$$

Then

$$
\begin{aligned}
& E_{t}\left[\frac{d V_{t, u}^{Y}}{V_{t, u}^{Y}}\right] \\
& =\frac{1}{v_{t, u}^{Y}}\left(\frac{\partial v_{t, u}^{Y}}{\partial t}+\frac{\partial v_{t, u}^{Y}}{\partial \omega_{t}} E_{t}\left[d \omega_{t}\right]+\frac{\partial v_{t, u}^{Y}}{\partial \nu_{1, t}} E_{t}\left[d \nu_{1, t}\right]+\frac{1}{2} \frac{\partial^{2} v_{t, u}^{Y}}{\partial \omega_{t}^{2}}\left(d \omega_{t}\right)^{2}+\frac{\partial^{2} v_{t, u}^{Y}}{\partial \omega_{t} \partial \nu_{1, t}} d \omega_{t} d \nu_{1, t}+\frac{1}{2} \frac{\partial^{2} v_{t, u}^{Y}}{\partial \nu_{1, t}^{2}}\left(d \nu_{1, t}\right)^{2}\right) \\
& +\mu_{Y} d t+\frac{1}{v_{t, u}^{Y}}\left(\frac{\partial v_{t, u}^{Y}}{\partial \omega_{t}} d \omega_{t}+\frac{\partial v_{t, u}^{Y}}{\partial \nu_{1, t}} d \nu_{1, t}\right) \frac{d Y_{t}}{Y_{t}} \\
& =\frac{1}{v_{t, u}^{Y}}\left(\frac{\partial v_{t, u}^{Y}}{\partial t}+\frac{\partial v_{t, u}^{Y}}{\partial \omega_{t}} \lambda_{x}\left(\bar{\omega}-\omega_{t}\right)+\frac{\partial v_{t, u}^{Y}}{\partial \nu_{1, t}} \nu_{1, t} \mu_{\nu_{1}, t}+\frac{1}{2} \frac{\partial^{2} v_{t, u}^{Y}}{\partial \omega_{t}^{2}} \sigma_{Y}^{2}+\frac{\partial^{2} v_{t, u}^{Y}}{\partial \omega_{t} \partial \nu_{1, t}} \sigma_{Y} \nu_{1, t} \sigma_{\nu_{1}, t}+\frac{1}{2} \frac{\partial^{2} v_{t, u}^{Y}}{\partial \nu_{1, t}^{2}} \nu_{1, t}^{2} \sigma_{\nu_{1, t}}^{2}\right) \\
& +\mu_{Y} d t+\frac{1}{v_{t, u}^{Y}}\left(\frac{\partial v_{t, u}^{Y}}{\partial \omega_{t}} \sigma_{Y}+\frac{\partial v_{t, u}^{Y}}{\partial \nu_{1, t}} \nu_{1, t} \sigma_{\nu_{1}, t}\right) \sigma_{Y},
\end{aligned}
$$

and

$$
-E_{t}\left[\frac{d V_{t, u}^{Y}}{V_{t, u}^{Y}} \frac{d \pi_{t}}{\pi_{t}}\right]=\left[\frac{1}{v_{t, u}^{Y}}\left(\frac{\partial v_{t, u}^{Y}}{\partial \omega_{t}} \sigma_{Y} d Z_{t}+\frac{\partial v_{t, u}^{Y}}{\partial \nu_{1, t}} \nu_{1, t} \sigma_{\nu_{1}, t}\right)+\sigma_{Y}\right] \theta_{t} d t
$$

Equation (A27) then follows from (A28).

When $\omega_{t} \rightarrow \infty$, current marginal utility tends to zero for Agent $k, k \in\{1,2\}$ (provided $\gamma_{k}>\frac{1}{\psi_{k}}$ ) However, $\omega$ is mean reverting and so future marginal utility will be higher. Consequently, the marginal rate of substitution is infinite and so the price-dividend ratio will be infinite. When $\omega_{t} \rightarrow$ $-\infty$, current marginal utility tends to $\infty$ for Agent $k, k \in\{1,2\}$, (provided $\gamma_{k}>\frac{1}{\psi_{k}}$ ). However, $\omega$ is mean reverting and so future marginal utility will be lower. Consequently, the marginal rate of substitution is zero and so the price-dividend ratio will be zero. Hence, $\lim _{\omega_{t} \rightarrow \infty} v_{t, u}^{Y}=\infty$ and $\lim _{\omega_{t} \rightarrow-\infty} v_{t, u}^{Y}=0$.

When $\nu_{k, t}=1$, the economy is populated solely by agents of Type $k$. Hence

$$
\begin{aligned}
v_{t, u}^{X} & =E_{t}\left[\frac{\hat{\pi}_{k, u}}{\hat{\pi}_{k, t}} \frac{X_{u}}{X_{t}}\right] \\
& =E_{t}\left[\frac{\xi_{k, u}}{\xi_{k, t}} e^{-\beta_{k}(u-t)} e^{\left(\gamma_{k}-\frac{1}{\psi_{k}}\right)\left(\omega_{u}-\omega_{t}\right)-\frac{1}{\psi_{k}}\left(y_{u}-y_{t}\right)} \frac{X_{u}}{X_{t}}\right] \\
& =e^{-\beta_{k}(u-t)} e^{-\left(\gamma_{k}-\frac{1}{\psi_{k}}\right) \omega_{t}+\frac{1}{\psi_{k}} y_{t}-x_{t}} E_{t}\left[\frac{\xi_{k, u}}{\xi_{k, t}} e^{\left(\gamma_{k}-\frac{1}{\psi_{k}}\right) \omega_{u}-\frac{1}{\psi_{k}} y_{u}+x_{u}}\right],
\end{aligned}
$$

where $x=\ln X$. 
We can show, after some algebra that

$$
\frac{\hat{\pi}_{k, u}}{\hat{\pi}_{k, u}} \frac{X_{u}}{X_{t}}=e^{-k_{k}(u-t)} \frac{M_{k, u}}{M_{k, t}} e^{-\left(\gamma_{k}-\frac{1}{\psi_{k}}\right)\left(\omega_{u}-\omega_{t}\right)}
$$

where

$$
k_{k}=\beta_{k}+\frac{1}{\psi_{k}} \mu_{Y, k}-\frac{1}{2} \frac{1}{\psi_{k}}\left(1+\frac{1}{\psi_{k}}\right) \sigma_{Y}^{2}+\frac{1}{\psi_{k}} \sigma_{X}^{s y s} \sigma_{Y}-\mu_{X, k},
$$

and $M_{k, t}$ is the following exponential martingale under $\mathbb{P}$ :

$$
\frac{d M_{k, t}}{M_{k, t}}=\sigma_{X}^{i d} d Z_{t}^{i d}+\left(\sigma_{X}^{s y s}+\sigma_{\xi, k}-\frac{1}{\psi_{k}} \sigma_{Y}\right) d Z_{t}, M_{k, t}=1 .
$$

We define the new probability measures $\hat{\mathbb{P}}^{k}$ on $(\Omega, \mathcal{F})$ via

$$
\hat{\mathbb{P}}^{k}(A)=E\left(1_{A} M_{k, T}\right), A \in \mathcal{F}_{T}, k \in\{1,2\} .
$$

Hence,

$$
\begin{aligned}
v_{t, u}^{X} & =E_{t}\left[e^{-k_{k}(u-t)} \frac{M_{k, u}}{M_{k, t}} e^{-\left(\gamma_{k}-\frac{1}{\psi_{k}}\right)\left(\omega_{u}-\omega_{t}\right)}\right] \\
& =e^{-k_{k}(u-t)} E_{t}^{k}\left[e^{-\left(\gamma_{k}-\frac{1}{\psi_{k}}\right)\left(\omega_{u}-\omega_{t}\right)}\right] .
\end{aligned}
$$

Define the moment generating function

$$
\mathcal{M}^{k}\left(\omega_{t}, u-t, a\right)=E_{t}^{k}\left[e^{a \omega_{u}}\right]
$$

Since $\omega$ is Gaussian, we have

$$
\begin{aligned}
\mathcal{M}^{k}\left(\omega_{t}, u-t, a\right) & =e^{a E_{t}^{k}\left[\omega_{u}\right]+\frac{1}{2} a^{2} \operatorname{Var}_{t}^{k}\left[\omega_{u}\right]} \\
& =e^{a\left[e^{-\lambda_{x}(u-t)} \omega_{t}+\left(1-e^{-\lambda_{x}(u-t)}\right) \hat{\omega}_{k}^{X}\right]+\frac{1}{2} a^{2} \frac{1-2 \lambda_{x}(u-t)}{2 \lambda_{x}} \sigma_{Y}^{2}},
\end{aligned}
$$

where

$$
\hat{\omega}_{k}^{X}=\frac{\mu_{Y, k}+\left(\sigma_{X}^{s y s}-\frac{1}{\psi_{k}} \sigma_{Y}\right) \sigma_{Y}-\frac{1}{2} \sigma_{Y}^{2}}{\lambda_{x}} .
$$

Hence,

$$
\begin{aligned}
v_{t, u}^{X} & =e^{-k_{k}(u-t)} e^{\left(\gamma_{k}-\frac{1}{\psi_{k}}\right) \omega_{t}} \mathcal{M}^{k}\left(\omega_{t}, u-t,-\left(\gamma_{k}-\frac{1}{\psi_{k}}\right)\right) \\
& =e^{-k_{k}(u-t)} e^{\left(\gamma_{k}-\frac{1}{\psi_{k}}\right) \omega_{t}} e^{-\left(\gamma_{k}-\frac{1}{\psi_{k}}\right)\left\{\left[e^{-\lambda_{x}(u-t)} \omega_{t}+\left(1-e^{-\lambda_{x}(u-t)}\right) \hat{\omega}_{k}^{X}\right]-\frac{1}{2}\left(\gamma_{k}-\frac{1}{\psi_{k}}\right) \frac{1-e^{-2 \lambda_{x}(u-t)}}{2 \lambda_{x}} \sigma_{Y}^{2}\right\}} \\
& =e^{-k_{k}(u-t)} e^{-\left(\gamma_{k}-\frac{1}{\psi_{k}}\right)\left[\left(1-e^{-\lambda_{x}(u-t)}\right)\left(\hat{\omega}_{k}^{X}-\omega_{t}\right)-\frac{1}{2}\left(\gamma_{k}-\frac{1}{\psi_{k}}\right) \frac{1-e^{-2 \lambda_{x}(u-t)}}{2 \lambda_{x}} \sigma_{Y}^{2}\right]} .
\end{aligned}
$$


Therefore,

$$
v_{t, u}^{Y}=e^{-k_{k}(u-t)} e^{-\left(\gamma_{k}-\frac{1}{\psi_{k}}\right)\left[\left(1-e^{-\lambda_{x}(u-t)}\right)\left(\hat{\omega}_{k}^{Y}-\omega_{t}\right)-\frac{1}{2}\left(\gamma_{k}-\frac{1}{\psi_{k}}\right) \frac{1-e^{-2 \lambda_{x}(u-t)}}{2 \lambda_{x}} \sigma_{Y}^{2}\right]},
$$

where now

$$
k_{k}=\beta_{k}+\frac{1}{\psi_{k}} \mu_{Y, k}-\frac{1}{2} \frac{1}{\psi_{k}}\left(1+\frac{1}{\psi_{k}}\right) \sigma_{Y}^{2}+\frac{1}{\psi_{k}} \sigma_{Y}^{2}-\mu_{Y, k}
$$

and

$$
\hat{\omega}_{k}^{Y}=\frac{\mu_{Y, k}+\left(1-\frac{1}{\psi_{k}}\right) \sigma_{Y}^{2}-\frac{1}{2} \sigma_{Y}^{2}}{\lambda_{x}} .
$$

Observe that the long-term yield of the risky security with price $v_{t, u}^{Y}$ is given by

$$
-\lim _{u \rightarrow \infty} \frac{1}{u-t} \ln v_{t, u}^{Y}=\beta_{k}+\frac{1}{\psi_{k}} \mu_{Y, k}-\frac{1}{2} \frac{1}{\psi_{k}}\left(1+\frac{1}{\psi_{k}}\right) \sigma_{Y}^{2}+\frac{1}{\psi_{k}} \sigma_{Y}^{2}-\mu_{Y, k}
$$

While we could solve (A27) numerically, an analytical approach would seem difficult, given the relatively complicated non-linear dependence of $\mu_{\nu_{1}, t}$ and $\sigma_{\nu_{1}, t}$ on $\nu_{1, t}$. Nevertheless, we can find an analytical solution by using Proposition 3. This relies on finding only the value of the claims $\phi_{n, 1, t, u}^{Y}$ and $\phi_{n, 2, t, u}^{Y}$ defined in Equations (29) and (30), which pay $Y_{u}$ at $u$ whenever $\frac{\hat{\pi}_{1, u}}{\hat{\pi}_{2, u}}>R$ and $\frac{\hat{\pi}_{1, u}}{\hat{\pi}_{2, u}}<R$, respectively. The proof uses the following Lemmas.

Lemma A2 (Values of $\phi_{n, 1, t}^{X}$ and $\phi_{n, 2, t}^{X}$ )

$$
\begin{aligned}
& \phi_{n, 1, t}^{X}=e^{-k_{1}(T-t)} e^{-a_{1} q_{t}-b_{1} \omega_{t}} \hat{E}_{t}^{1}\left[e^{a_{1} q_{T}+b_{1} \omega_{T}} 1_{\left\{q_{T}+d_{\omega} \omega_{T}>\rho\right\}}\right] \\
& \phi_{n, 2, t}^{X}=e^{-k_{2}(T-t)} e^{-a_{2} q_{t}-b_{2} \omega_{t}} \hat{E}_{t}^{2}\left[e^{a_{2} q_{T}+b_{2} \omega_{T}} 1_{\left\{q_{T}+d_{\omega} \omega_{T}<\rho\right\}}\right],
\end{aligned}
$$

where

$$
\begin{aligned}
& \hat{r}_{1}=\beta_{1}+\frac{1}{\psi_{1}} \mu_{Y, 1}-\frac{1}{2} \frac{1}{\psi_{1}}\left(1+\frac{1}{\psi_{1}}\right) \sigma_{Y}^{2} \\
& \hat{r}_{2}=\beta_{2}+\frac{1}{\psi_{2}} \mu_{Y, 2}-\frac{1}{2} \frac{1}{\psi_{2}}\left(1+\frac{1}{\psi_{2}}\right) \sigma_{Y}^{2} \\
& \hat{k}_{1}=\hat{r}_{1}+\frac{1}{\psi_{1}} \sigma_{X}^{\text {sys }} \sigma_{Y}-\mu_{X, 1} \\
& \hat{k}_{2}=\hat{r}_{2}+\frac{1}{\psi_{2}} \sigma_{X}^{\text {sys }} \sigma_{Y}-\mu_{X, 2} \\
& a_{1}=-\frac{n}{\gamma_{2}} \\
& b_{1}=-\left[\frac{n}{\gamma_{2}}\left(\gamma_{2}-\frac{1}{\psi_{2}}\right)+\left(1-\frac{n}{\gamma_{2}}\right)\left(\gamma_{1}-\frac{1}{\psi_{1}}\right)\right]
\end{aligned}
$$




$$
\begin{aligned}
a_{2} & =\frac{n}{\gamma_{1}} \\
b_{2} & =-\left[\left(1-\frac{n}{\gamma_{2}}\right)\left(\gamma_{2}-\frac{1}{\psi_{2}}\right)+\frac{n}{\gamma_{1}}\left(\gamma_{1}-\frac{1}{\psi_{1}}\right)\right] \\
\rho & =\ln R,
\end{aligned}
$$

and $\hat{E}_{t}^{k}[\cdot]$ is the date-t conditional expectation operator with respect to the probability measure $\hat{\mathbb{P}}^{k}$ defined in (A30).

\section{Proof of Lemma A2}

Recall that

$$
\begin{aligned}
& \phi_{n, 1, t, T}^{X}=E_{t}\left[\frac{\hat{\pi}_{1, T}^{1-\frac{n}{\gamma_{2}}} \hat{\pi}_{2, T}^{\frac{n}{\gamma_{2}}}}{\hat{\pi}_{1, t}^{1-\frac{n}{\gamma_{2}}} \hat{\pi}_{2, t}^{\frac{n}{\gamma_{2}}}} \frac{X_{T}}{X_{t}} 1_{\left\{\frac{\hat{\pi}_{1, T}}{\hat{\pi}_{2, T}}>R\right\}}\right], n \in \mathbb{N}_{0}, \\
& \phi_{n, 2, t, T}^{X}=E_{t}\left[\frac{\hat{\pi}_{1, T}^{\frac{n}{\gamma_{1}}} \hat{\pi}_{2, T}^{1-\frac{n}{\gamma_{1}}}}{\hat{\pi}_{1, t}^{\frac{n}{\gamma_{1}}} \hat{\pi}_{2, t}^{1-\frac{n}{\gamma_{1}}}} \frac{X_{T}}{X_{t}} 1_{\left\{\hat{\pi}_{1, T}<R\right\}}\right], n \in \mathbb{N}_{0} .
\end{aligned}
$$

Simplifying (A37) gives

$$
\begin{aligned}
\phi_{n, 1, t, T}^{X} & \left.=E_{t}\left[\frac{\hat{\pi}_{1, T}^{1-\frac{n}{\gamma_{2}}} \hat{\pi}_{2, T}^{\frac{n}{\gamma_{2}}}}{\hat{\pi}_{1, t}^{1-\frac{n}{\gamma_{2}}} \hat{\pi}_{2, t}^{\frac{n}{\gamma_{2}}}} \frac{X_{T}}{X_{t}} 1_{\{} \frac{\hat{\pi}_{1, T}}{\hat{\pi}_{2, T}}>R\right\}\right] \\
& \left.=E_{t}\left[\left(\frac{\hat{\pi}_{1, T} / \hat{\pi}_{2, T}}{\hat{\pi}_{1, t} / \hat{\pi}_{2, t}}\right)^{-\frac{n}{\gamma_{2}}} \frac{\hat{\pi}_{1, T}}{\hat{\pi}_{1, t}} \frac{X_{T}}{X_{t}} 1 \ln \left(\frac{\hat{\pi}_{1, T}}{\hat{\pi}_{2, T}}\right)>\rho\right\}\right]
\end{aligned}
$$

where $\rho$ is defined in (A36). Simplifying (A38) gives

$$
\begin{aligned}
\phi_{n, 2, t, T}^{X} & \left.=E_{t}\left[\frac{\hat{\pi}_{1, T}^{\frac{n}{\gamma_{1}}} \hat{\pi}_{2, T}^{1-\frac{n}{\gamma_{1}}}}{\hat{\pi}_{1, t}^{\frac{n}{\gamma_{1}}} \hat{\pi}_{2, t}^{1-\frac{n}{\gamma_{1}}}} \frac{X_{T}}{X_{t}} 1_{\{} \hat{\frac{\pi}{1, T}}_{\hat{\pi}_{2, T}}<R\right\}\right] \\
& =E_{t}\left[\left(\frac{\hat{\pi}_{1, T} / \hat{\pi}_{2, T}}{\hat{\pi}_{1, t} / \hat{\pi}_{2, t}}\right)^{\frac{n}{\gamma_{1}}} \frac{\hat{\pi}_{2, T}}{\hat{\pi}_{2, t}} \frac{X_{T}}{X_{t}}\left\{\ln \left(\frac{\hat{\pi}_{1, T}}{\hat{\pi}_{2, T}}\right)<\rho\right\}\right] .
\end{aligned}
$$

Note that

$$
\frac{\hat{\pi}_{k, u}}{\hat{\pi}_{k, u}} \frac{X_{u}}{X_{t}}=e^{-k_{k}(u-t)} \frac{M_{k, u}}{M_{k, t}} e^{-\left(\gamma_{k}-\frac{1}{\psi_{k}}\right)\left(\omega_{T}-\omega_{t}\right)}
$$

where $k_{k}, k \in\{1,2\}$ are defined in (A32) and (A33), and $M_{k, t}, k \in\{1,2\}$, is defined in (A29). Hence

$$
\phi_{n, 1, t, T}^{X}=E_{t}\left[\left(\frac{\hat{\pi}_{1, T} / \hat{\pi}_{2, T}}{\hat{\pi}_{1, t} / \hat{\pi}_{2, t}}\right)^{-\frac{n}{\gamma_{2}}} e^{-k_{1}(T-t)} \frac{M_{1, T}}{M_{1, t}} e^{-\left(\gamma_{1}-\frac{1}{\psi_{1}}\right)\left(\omega_{T}-\omega_{t}\right)} 1_{\left\{q_{T}+d_{\omega} \omega_{T}>\rho\right\}}\right]
$$




$$
\phi_{n, 2, t, T}^{X}=E_{t}\left[\left(\frac{\hat{\pi}_{1, T} / \hat{\pi}_{2, T}}{\hat{\pi}_{1, t} / \hat{\pi}_{2, t}}\right)^{\frac{n}{\gamma_{1}}} e^{-k_{2}(T-t)} \frac{M_{2, T}}{M_{2, t}} e^{-\left(\gamma_{2}-\frac{1}{\psi_{2}}\right)\left(\omega_{T}-\omega_{t}\right)} 1_{\left\{q_{T}+d_{\omega} \omega_{T}<\rho\right\}}\right] .
$$

Therefore,

$$
\begin{aligned}
\phi_{n, 1, t}^{X} & =\hat{E}_{t}^{1}\left[\left(\frac{\hat{\pi}_{1, T} / \hat{\pi}_{2, T}}{\hat{\pi}_{1, t} / \hat{\pi}_{2, t}}\right)^{-\frac{n}{\gamma_{2}}} e^{-k_{1}(T-t)} e^{-\left(\gamma_{1}-\frac{1}{\psi_{1}}\right)\left(\omega_{T}-\omega_{t}\right)} 1_{\left\{q_{T}+d_{\omega} \omega_{T}>\rho\right\}}\right] \\
& =e^{-k_{1}(T-t)} e^{-a_{1} q_{t}-b_{1} \omega_{t}} \hat{E}_{t}^{1}\left[e^{a_{1} q_{T}+b_{1} \omega_{T}} 1_{\left\{q_{T}+d_{\omega} \omega_{T}>\rho\right\}}\right]
\end{aligned}
$$

and

$$
\begin{aligned}
\phi_{n, 2, t}^{X} & =\hat{E}_{t}^{2}\left[\left(\frac{\hat{\pi}_{1, T} / \hat{\pi}_{2, T}}{\hat{\pi}_{1, t} / \hat{\pi}_{2, t}}\right)^{\frac{n}{\gamma_{1}}} e^{-k_{2}(T-t)} e^{-\left(\gamma_{2}-\frac{1}{\psi_{2}}\right)\left(\omega_{T}-\omega_{t}\right)} 1_{\left\{q_{T}+d_{\omega} \omega_{T}<\rho\right\}}\right] \\
& =e^{-k_{2}(T-t)} e^{-a_{2} q_{t}-b_{2} \omega_{t}} \hat{E}_{t}^{2}\left[e^{a_{2} q_{T}+b_{2} \omega_{T}} 1_{\left\{q_{T}+d_{\omega} \omega_{T}<\rho\right\}}\right]
\end{aligned}
$$

where $a_{1}, b_{1}, a_{2}$, and $b_{2}$ are defined in (A34)-(A35).

We shall need some basic definitions for Fourier Transforms in order to prove Lemma A3.

Definition A4 For an integrable function $f: \mathbb{R} \rightarrow \mathbb{C}$, its Fourier Transform is given by

$$
\hat{f}(x)=\int_{-\infty}^{\infty} e^{i k x} f(k) d k, \forall x \in \mathbb{R}
$$

We shall use the notation

$$
\mathcal{F}[f(k), x]=\int_{-\infty}^{\infty} e^{i k x} f(k) d k .
$$

Under certain conditions (see, for example Friedlander and Joshi (1999)) $f(k)$ can be reconstructed from $\mathcal{F}[f(k), x]$ via the inverse Fourier transform

$$
f(k)=\frac{1}{2 \pi} \int_{-\infty}^{\infty} e^{-i k x} \hat{f}(x) d x
$$

for which we use the notation

$$
\mathcal{F}^{-1}[\hat{f}(x), k]=\frac{1}{2 \pi} \int_{-\infty}^{\infty} e^{-i k x} \hat{f}(x) d x .
$$

The following Lemma provides a closed form expression for the moment generating function of the 2-dimensional affine process, $\left(q_{t}, \omega_{t}\right)$, under the probability measure $\hat{\mathbb{P}}^{k}$.

\section{Lemma A3 (Moment generating function)}

$$
\hat{E}_{t}^{k}\left[e^{a_{k} q_{T}+b_{k} \omega_{T}} 1_{\left\{q_{T}+d_{\omega} \omega_{T}>\rho\right\}}\right]=\mathcal{M}_{k}^{X}\left(q_{t}, \omega_{t}, T-t, a_{k}, b_{k}\right) \Phi\left(\frac{B_{k}^{X}\left(q_{t}, \omega_{t}, T-t\right)-\rho}{\sqrt{2 A_{k}\left(q_{t}, \omega_{t}, T-t\right)}}\right),
$$


where the moment generating function, $\mathcal{M}_{k}^{X}\left(q_{t}, \omega_{t}, T-t, a, b\right)$, defined by

$$
\mathcal{M}_{k}^{X}\left(q_{t}, \omega_{t}, T-t, a, b\right)=\hat{E}_{t}^{k}\left[e^{a q_{T}+b \omega_{T}}\right]
$$

is given by

$$
\begin{aligned}
\mathcal{M}_{k}^{X}\left(q_{t}, \omega_{t}, T-t, a, b\right) & =\exp \left\{a\left[q_{t}+\hat{\mu}_{q, k}^{X}(T-t)\right]+b\left[e^{-\lambda_{x}(T-t)} \omega_{t}+\left(1-e^{-\lambda_{x}(T-t)}\right) \hat{\omega}_{k}^{X}\right]\right. \\
& \left.+\frac{1}{2} a^{2} \sigma_{q}^{2}(T-t)+a b \frac{1-e^{-\lambda_{x}(T-t)}}{\lambda_{x}} \sigma_{q} \sigma_{Y}+\frac{1}{2} b^{2} \frac{1-e^{-2 \lambda_{x}(T-t)}}{2 \lambda_{x}} \sigma_{Y}^{2}\right\},
\end{aligned}
$$

and

$$
\begin{aligned}
A_{k}\left(q_{t}, \omega_{t}, T-t\right) & =\frac{1}{2}\left[\sigma_{q}^{2}(T-t)+2 d_{\omega} \frac{1-e^{-\lambda_{x}(T-t)}}{\lambda_{x}} \sigma_{q} \sigma_{Y}+d_{\omega}^{2} \frac{1-e^{-2 \lambda_{x}(T-t)}}{2 \lambda_{x}} \sigma_{Y}^{2}\right] \\
B_{k}^{X}\left(q_{t}, \omega_{t}, T-t\right) & =q_{t}+\hat{\mu}_{q, k}^{X}(T-t)+d_{\omega}\left[e^{-\lambda_{x}(T-t)} \omega_{t}+\left(1-e^{-\lambda_{x}(T-t)}\right) \hat{\omega}_{k}^{X}\right] \\
& +a_{k} \sigma_{q}^{2}(T-t)+\left(b_{k}+a_{k} d_{\omega}\right) \frac{1-e^{-\lambda_{x}(T-t)}}{\lambda_{x}} \sigma_{q} \sigma_{Y}+b_{k} d_{\omega} \frac{1-e^{-2 \lambda_{x}(T-t)}}{2 \lambda_{x}} \sigma_{Y}^{2},
\end{aligned}
$$

where

$$
\begin{aligned}
\mu_{q} & =\left(\beta_{2}-\beta_{1}\right)+\frac{1}{2}\left(\sigma_{\xi, 2}^{2}-\sigma_{\xi, 1}^{2}\right)+\left(\frac{1}{\psi_{2}}-\frac{1}{\psi_{1}}\right)\left(\mu_{Y}-\frac{1}{2} \sigma_{Y}^{2}\right), \\
\sigma_{q} & =\sigma_{\xi, 1}-\sigma_{\xi, 2}+\left(\frac{1}{\psi_{2}}-\frac{1}{\psi_{1}}\right) \sigma_{Y}, \\
\hat{\mu}_{q, k}^{X} & =\mu_{q}+\left(\sigma_{X}^{s y s}+\sigma_{\xi, k}-\frac{1}{\psi_{k}} \sigma_{Y}\right) \sigma_{q} \\
\hat{\omega}_{k}^{X} & =\frac{\mu_{Y, k}-\frac{1}{2} \sigma_{Y}^{2}+\left(\sigma_{X}^{s y s}-\frac{1}{\psi_{k}} \sigma_{Y}\right) \sigma_{Y}}{\lambda_{x}} \\
d_{\omega} & =\left(\gamma_{2}-\frac{1}{\psi_{2}}\right)-\left(\gamma_{1}-\frac{1}{\psi_{1}}\right) .
\end{aligned}
$$

\section{Proof of Lemma A3}

First we prove $(\mathrm{A} 43)$. Because $\left(q_{T}, \omega_{T}\right)$ is Gaussian, we have

$$
\mathcal{M}_{k}^{X}\left(q_{t}, \omega_{t}, T-t, a, b\right)=e^{\hat{E}^{k}\left[a q_{T}+b \omega_{T}\right]+\frac{1}{2} \widehat{\operatorname{Var}}_{t}^{k}\left[a q_{T}+b \omega_{T}\right]},
$$

where $\widehat{\operatorname{Var}}_{t}^{k}$ is the time- $t$ conditional variance operator under the probability measure $\widehat{\mathbb{P}}^{k}$. Note that

$$
\begin{aligned}
\hat{E}^{k}\left[a q_{T}+b \omega_{T}\right] & =a \hat{E}^{k}\left[q_{T}\right]+b \hat{E}^{k}\left[\omega_{T}\right] \\
& =a\left[q_{t}+\hat{\mu}_{q, k}^{X}(T-t)\right]+b\left[e^{-\lambda_{x}(T-t)} \omega_{t}+\left(1-e^{-\lambda_{x}(T-t)}\right) \hat{\omega}_{k}^{X}\right]
\end{aligned}
$$


and

$$
\begin{aligned}
\frac{1}{2} \widehat{\operatorname{Var}}_{t}^{k}\left[a q_{T}+b \omega_{T}\right] & =\frac{1}{2} a^{2}{\widehat{\operatorname{Var}_{t}}}_{t}^{k}\left[q_{T}\right]+a b \widehat{\operatorname{Cov}}_{t}^{k}\left[q_{T}, \omega_{T}\right]+\frac{1}{2} b^{2}{\widehat{\operatorname{Var}_{t}}}_{t}^{k}\left[\omega_{T}\right] \\
& =\frac{1}{2} a^{2} \sigma_{q}^{2}(T-t)+a b \frac{1-e^{-\lambda_{x}(T-t)}}{\lambda_{x}} \sigma_{q} \sigma_{Y}+\frac{1}{2} b^{2} \frac{1-e^{-2 \lambda_{x}(T-t)}}{2 \lambda_{x}} \sigma_{Y}^{2}
\end{aligned}
$$

where the long-run mean of $\omega$ under the probability measure $\hat{\mathbb{P}}^{k}$ is given by $\hat{\omega}_{k}^{X}$, defined in (A46). Hence,

$$
\begin{aligned}
\mathcal{M}_{k}^{X}\left(q_{t}, \omega_{t}, T-t, a, b\right) & =\exp \left\{a\left[q_{t}+\hat{\mu}_{q, k}^{X}(T-t)\right]+b\left[e^{-\lambda_{x}(T-t)} \omega_{t}+\left(1-e^{-\lambda_{x}(T-t)}\right) \hat{\omega}_{k}^{X}\right]\right. \\
& \left.+\frac{1}{2} a^{2} \sigma_{q}^{2}(T-t)+a b \frac{1-e^{-\lambda_{x}(T-t)}}{\lambda_{x}} \sigma_{q} \sigma_{Y}+\frac{1}{2} b^{2} \frac{1-e^{-2 \lambda_{x}(T-t)}}{2 \lambda_{x}} \sigma_{Y}^{2}\right\}
\end{aligned}
$$

Our proof of (A41) relies on using Fourier transforms (see Heston (1993) and Duffie, Pan, and Singleton (2000)). Taking the Fourier transform of $\hat{E}_{t}^{k}\left[e^{a_{k} q_{T}+b_{k} \omega_{T}} 1_{\left\{q_{T}+d_{\omega} \omega_{T}>\rho\right\}}\right]$ gives (see (A39) and (A40) for relevant definitions)

$$
\begin{aligned}
\mathcal{F}\left[\hat{E}_{t}^{k}\left[e^{a_{k} q_{T}+b_{k} \omega_{T}} 1_{\left\{q_{T}+d_{\omega} \omega_{T}>\rho\right\}}\right], x\right] & =\hat{E}_{t}^{k}\left[e^{a_{k} q_{T}+b_{k} \omega_{T}} \mathcal{F}\left[1_{\left\{q_{T}+d_{\omega} \omega_{T}>\rho\right\}}, x\right]\right] \\
& =\hat{E}_{t}^{k}\left[e^{a_{k} q_{T}+b_{k} \omega_{T}} \mathcal{F}\left[\theta\left(q_{T}+d_{\omega} \omega_{T}-\rho\right), x\right]\right],
\end{aligned}
$$

where $\theta(z)$ is the Heaviside step function, defined by

$$
\theta(z)= \begin{cases}0, & z<0 \\ \frac{1}{2}, & z=0 \\ 1, & z>0\end{cases}
$$

Using the standard result that

$$
\mathcal{F}\left[\theta\left(q_{T}+d_{\omega} \omega_{T}-\rho\right), x\right]=\frac{e^{i x\left(q_{T}+d_{\omega} \omega_{T}\right)}}{i x}+\pi \delta(x),
$$

where $\delta(z)$ is the Dirac-delta function. It follows that

$$
\begin{aligned}
\mathcal{F}\left[\hat{E}_{t}^{k}\left[e^{a_{k} q_{T}+b_{k} \omega_{T}} 1_{\left\{q_{T}+d_{\omega} \omega_{T}>\rho\right\}}\right], x\right] & =\hat{E}_{t}^{k}\left[e^{a_{k} q_{T}+b_{k} \omega_{T}}\left(\frac{\left.e^{i x\left(q_{T}+d_{\omega} \omega_{T}\right.}\right)}{i x}+\pi \delta(x)\right)\right] \\
& =\frac{\mathcal{M}_{k}^{X}\left(q_{t}, \omega_{t}, T-t, a_{k}+i x, b_{k}+i x d_{\omega}\right)}{i x} \\
& +\pi \delta(x) \mathcal{M}_{k}^{X}\left(q_{t}, \omega_{t}, T-t, a_{k}, b_{k}\right),
\end{aligned}
$$

where $\mathcal{M}_{k}^{X}\left(q_{t}, \omega_{t}, T-t, a, b\right)$ is defined in (A42). Taking the inverse Fourier transform, we obtain

$$
\hat{E}_{t}^{k}\left[e^{a_{k} q_{T}+b_{k} \omega_{T}} 1_{\left\{q_{T}+d_{\omega} \omega_{T}>\rho\right\}}\right]=\mathcal{F}^{-1}\left[\frac{\mathcal{M}_{k}^{X}\left(q_{t}, \omega_{t}, T-t, a_{k}+i x, b_{k}+i x d_{\omega}\right)}{i x}, \rho\right]
$$




$$
\begin{aligned}
& +\pi \mathcal{F}^{-1}[\delta(x), \rho] \mathcal{M}_{k}^{X}\left(q_{t}, \omega_{t}, T-t, a_{k}, b_{k}\right) \\
& =\frac{1}{2 \pi} \int_{-\infty}^{\infty} e^{-i \rho x} \frac{\mathcal{M}_{k}^{X}\left(q_{t}, \omega_{t}, T-t, a_{k}+i x, b_{k}+i x d_{\omega}\right)}{i x} d x \\
& +\pi \mathcal{M}_{k}^{X}\left(q_{t}, \omega_{t}, T-t, a_{k}, b_{k}\right) \frac{1}{2 \pi} \int_{-\infty}^{\infty} e^{-i \rho x} \delta(x) d x \\
& =\frac{1}{2 \pi} \int_{-\infty}^{\infty} e^{-i \rho x} \frac{\mathcal{M}_{k}^{X}\left(q_{t}, \omega_{t}, T-t, a_{k}+i x, b_{k}+i x d_{\omega}\right)}{i x} d x \\
& +\frac{1}{2} \mathcal{M}_{k}^{X}\left(q_{t}, \omega_{t}, T-t, a_{k}, b_{k}\right),
\end{aligned}
$$

where we have used the standard result that

$$
\int_{-\infty}^{\infty} e^{-i \rho x} \delta(x) d x=1
$$

We now show that

$$
\begin{aligned}
& \int_{-\infty}^{\infty} e^{-i \rho x} \frac{\mathcal{M}_{k}^{X}\left(q_{t}, \omega_{t}, T-t, a_{k}+i x, b_{k}+i x d_{\omega}\right)}{i x} d x \\
& =2 \mathcal{M}_{k}^{X}\left(q_{t}, \omega_{t}, T-t, a_{k}, b_{k}\right) \int_{0}^{\infty} e^{-A_{k}\left(q_{t}, \omega_{t}, T-t\right) x^{2}} \frac{\sin \left[\left(B_{k}^{X}\left(q_{t}, \omega_{t}, T-t\right)-\rho\right) x\right]}{x} d x
\end{aligned}
$$

where $A_{k}\left(q_{t}, \omega_{t}, T-t\right)$ and $B_{k}^{X}\left(q_{t}, \omega_{t}, T-t\right)$ are defined in (A44) and (A45), respectively. We start by observing that

$$
\mathcal{M}_{k}^{X}\left(q_{t}, \omega_{t}, T-t, a_{k}+i x, b_{k}+i x d_{\omega}\right)=\mathcal{M}_{k}^{X}\left(q_{t}, \omega_{t}, T-t, a_{k}, b_{k}\right) e^{-A_{k}\left(q_{t}, \omega_{t}, T-t\right) x^{2}+i B_{k}^{X}\left(q_{t}, \omega_{t}, T-t\right) x} .
$$

Hence,

$$
\begin{aligned}
& \int_{-\infty}^{\infty} e^{-i \rho x} \frac{\mathcal{M}_{k}^{X}\left(q_{t}, \omega_{t}, T-t, a_{k}+i x, b_{k}+i x d_{\omega}\right)}{i x} d x \\
& =\mathcal{M}_{k}^{X}\left(q_{t}, \omega_{t}, T-t, a_{k}, b_{k}\right) \int_{-\infty}^{\infty} e^{-A_{k}\left(q_{t}, \omega_{t}, T-t\right) x^{2}} \frac{e^{i\left(B_{k}^{X}\left(q_{t}, \omega_{t}, T-t\right)-\rho\right) x}}{i x} d x \\
& =\mathcal{M}_{k}^{X}\left(q_{t}, \omega_{t}, T-t, a_{k}, b_{k}\right) \\
& \times \int_{-\infty}^{\infty} e^{-A_{k}\left(q_{t}, \omega_{t}, T-t\right) x^{2}} \frac{\cos \left[\left(B_{k}^{X}\left(q_{t}, \omega_{t}, T-t\right)-\rho\right) x\right]+i \sin \left[\left(B_{k}^{X}\left(q_{t}, \omega_{t}, T-t\right)-\rho\right) x\right]}{i x} d x
\end{aligned}
$$

Since $e^{-A_{k}\left(q_{t}, \omega_{t}, T-t\right) x^{2}} \frac{\cos \left[\left(B_{k}^{X}\left(q_{t}, \omega_{t}, T-t\right)-\rho\right) x\right]}{x}$ and $e^{-A_{k}\left(q_{t}, \omega_{t}, T-t\right) x^{2}} \frac{\sin \left[\left(B_{k}^{X}\left(q_{t}, \omega_{t}, T-t\right)-\rho\right) x\right]}{x}$ are odd and even functions of $x$, respectively, it follows that

$$
\begin{aligned}
& \int_{-\infty}^{\infty} e^{-A_{k}\left(q_{t}, \omega_{t}, T-t\right) x^{2}} \frac{\cos \left[\left(B_{k}^{X}\left(q_{t}, \omega_{t}, T-t\right)-\rho\right) x\right]+i \sin \left[\left(B_{k}^{X}\left(q_{t}, \omega_{t}, T-t\right)-\rho\right) x\right]}{i x} d x \\
& =2 \int_{0}^{\infty} e^{-A_{k}\left(q_{t}, \omega_{t}, T-t\right) x^{2}} \frac{\sin \left[\left(B_{k}^{X}\left(q_{t}, \omega_{t}, T-t\right)-\rho\right) x\right]}{x} d x .
\end{aligned}
$$


Hence, (A48) follows from (A49).

Together with Equation (A48), (A47) implies that

$$
\begin{aligned}
\hat{E}_{t}^{k}\left[e^{a_{k} q_{T}+b_{k} \omega_{T}} 1_{\left\{q_{T}+d_{\omega} \omega_{T}>\rho\right\}}\right] & =\frac{1}{\pi} \int_{0}^{\infty} e^{-A_{k}\left(q_{t}, \omega_{t}, T-t\right) x^{2}} \frac{\sin \left[\left(B_{k}^{X}\left(q_{t}, \omega_{t}, T-t\right)-\rho\right) x\right]}{x} d x \\
& +\frac{1}{2} \mathcal{M}_{k}^{X}\left(q_{t}, \omega_{t}, T-t, a_{k}, b_{k}\right) .
\end{aligned}
$$

Since

$$
\int_{0}^{\infty} e^{-A_{k}\left(q_{t}, \omega_{t}, T-t\right) x^{2}} \frac{\sin \left[\left(B_{k}^{X}\left(q_{t}, \omega_{t}, T-t\right)-\rho\right) x\right]}{x} d x=\frac{1}{2} \pi\left[2 \Phi\left(\frac{B_{k}^{X}\left(q_{t}, \omega_{t}, T-t\right)-\rho}{\sqrt{2 A_{k}\left(q_{t}, \omega_{t}, T-t\right)}}\right)-1\right]
$$

where $\Phi(\cdot)$ is the standard cumulative normal distribution function (a result which can be obtained using Mathematica), (A50) implies (A41).

To prove Proposition 8, our first step is to use Lemma A2; we then use Lemma A3 to simplify (A37) and (A38):

$$
\hat{E}_{t}^{1}\left[e^{a_{1} q_{T}+b_{1} \omega_{T}} 1_{\left\{q_{T}+d_{\omega} \omega_{T}>\rho\right\}}\right]=\mathcal{M}_{1}^{X}\left(q_{t}, \omega_{t}, T-t, a_{1}, b_{1}\right) \Phi\left(\frac{B_{1}^{X}\left(q_{t}, \omega_{t}, T-t\right)-\rho}{\sqrt{2 A_{1}\left(q_{t}, \omega_{t}, T-t\right)}}\right)
$$

and

$$
\begin{aligned}
\hat{E}_{t}^{2}\left[e^{a_{2} q_{T}+b_{2} \omega_{T}} 1_{\left\{q_{T}+d_{\omega} \omega_{T}<\rho\right\}}\right] & =\hat{E}_{t}^{2}\left[e^{a_{2} q_{T}+b_{2} \omega_{T}}\left(1-1_{\left\{q_{T}+d_{\omega} \omega_{T}>\rho\right\}}\right)\right] \\
& =\hat{E}_{t}^{2}\left[e^{a_{2} q_{T}+b_{2} \omega_{T}}\right]-\hat{E}_{t}^{2}\left[e^{a_{2} q_{T}+b_{2} \omega_{T}} 1_{\left\{q_{T}+d_{\omega} \omega_{T}>\rho\right\}}\right] \\
& =\mathcal{M}_{2}^{X}\left(q_{t}, \omega_{t}, T-t, a_{2}, b_{2}\right)\left[1-\Phi\left(\frac{B_{2}^{X}\left(q_{t}, \omega_{t}, T-t\right)-\rho}{\sqrt{2 A_{2}\left(q_{t}, \omega_{t}, T-t\right)}}\right)\right] \\
& =\mathcal{M}_{2}^{X}\left(q_{t}, \omega_{t}, T-t, a_{2}, b_{2}\right) \Phi\left(\frac{\rho-B_{2}^{X}\left(q_{t}, \omega_{t}, T-t\right)}{\sqrt{2 A_{2}\left(q_{t}, \omega_{t}, T-t\right)}}\right) .
\end{aligned}
$$

Hence,

$$
\begin{aligned}
& \phi_{n, 1, t, T}^{X}=e^{-k_{1}(T-t)} e^{-a_{1} q_{t}-b_{1} \omega_{t}} \mathcal{M}_{1}^{X}\left(q_{t}, \omega_{t}, T-t, a_{1}, b_{1}\right) \Phi\left(\frac{B_{1}^{X}\left(q_{t}, \omega_{t}, T-t\right)-\rho}{\sqrt{2 A_{1}\left(q_{t}, \omega_{t}, T-t\right)}}\right) \\
& \phi_{n, 2, t, T}^{X}=e^{-k_{2}(T-t)} e^{-a_{2} q_{t}-b_{2} \omega_{t}} \mathcal{M}_{2}^{X}\left(q_{t}, \omega_{t}, T-t, a_{2}, b_{2}\right) \Phi\left(\frac{\rho-B_{2}^{X}\left(q_{t}, \omega_{t}, T-t\right)}{\sqrt{2 A_{2}\left(q_{t}, \omega_{t}, T-t\right)}}\right) .
\end{aligned}
$$

We know that

$$
q_{t}=\ln \frac{\hat{\pi}_{1, t}}{\hat{\pi}_{2, t}}-d_{\omega} \omega_{t}=\ln \left[\frac{\left(1-\nu_{1, t}\right)^{-\gamma_{2}}}{\nu_{1, t}^{-\gamma_{1}}}\right]-d_{\omega} \omega_{t}
$$


and so

$$
\begin{aligned}
& \phi_{n, 1, t, T}^{X}=e^{-k_{1}(T-t)-\left(b_{1}-a_{1} d_{\omega}\right) \omega_{t}}\left(\frac{\nu_{2, t}^{\gamma_{2}}}{\nu_{1, t}^{\gamma_{1}}}\right)^{a_{1}} \mathcal{M}_{1}^{X}\left(q_{t}, \omega_{t}, T-t, a_{1}, b_{1}\right) \Phi\left(\frac{B_{1}^{X}\left(q_{t}, \omega_{t}, T-t\right)-\rho}{\sqrt{2 A_{1}\left(q_{t}, \omega_{t}, T-t\right)}}\right) \\
& \phi_{n, 2, t, T}^{X}=e^{-k_{2}(T-t)-\left(b_{2}-a_{2} d_{\omega}\right) \omega_{t}}\left(\frac{\nu_{2, t}^{\gamma_{2}}}{\nu_{1, t}^{\gamma_{1}}}\right)^{a_{2}} \mathcal{M}_{2}^{X}\left(q_{t}, \omega_{t}, T-t, a_{2}, b_{2}\right) \Phi\left(\frac{\rho-B_{2}^{X}\left(q_{t}, \omega_{t}, T-t\right)}{\sqrt{2 A_{2}\left(q_{t}, \omega_{t}, T-t\right)}}\right) .
\end{aligned}
$$

Simplifying the above expressions gives

$$
\begin{aligned}
\phi_{n, 1, t, T}^{X} & =e^{-k_{1}(T-t)+b_{1}\left(\omega_{t}-\hat{\omega}_{k}^{X}\right)\left[1-e^{-\lambda_{x}(T-t)}\right]} \exp \left\{a_{1} \hat{\mu}_{q, 1}^{X}(T-t)+\frac{1}{2} a_{1}^{2} \sigma_{q}^{2}(T-t)\right. \\
& \left.+a_{1} b_{1} \frac{1-e^{-\lambda_{x}(T-t)}}{\lambda_{x}} \sigma_{q} \sigma_{X}^{s y s}+\frac{1}{2} b_{1}^{2} \frac{1-e^{-2 \lambda_{x}(T-t)}}{2 \lambda_{x}} \sigma_{X}^{2}\right\} \Phi\left(\frac{B_{1}^{X}\left(q_{t}, \omega_{t}, T-t\right)-\rho}{\sqrt{2 A_{1}\left(q_{t}, \omega_{t}, T-t\right)}}\right), \\
\phi_{n, 2, t, T}^{X} & =e^{-k_{2}(T-t)+b_{2}\left(\omega_{t}-\hat{\omega}_{k}^{X}\right)\left[1-e^{-\lambda_{x}(T-t)}\right]} \exp \left\{a_{2} \hat{\mu}_{q, 2}^{X}(T-t)+\frac{1}{2} a_{2}^{2} \sigma_{q}^{2}(T-t)\right. \\
& \left.+a_{2} b_{2} \frac{1-e^{-\lambda_{x}(T-t)}}{\lambda_{x}} \sigma_{q} \sigma_{X}^{s y s}+\frac{1}{2} b_{2}^{2} \frac{1-e^{-2 \lambda_{x}(T-t)}}{2 \lambda_{x}} \sigma_{X}^{2}\right\} \Phi\left(\frac{\rho-B_{2}^{X}\left(q_{t}, \omega_{t}, T-t\right)}{\sqrt{2 A_{2}\left(q_{t}, \omega_{t}, T-t\right)}}\right) .
\end{aligned}
$$

Replacing the cashflow $X$ with the aggregate endowment $Y$ gives (59) and (60).

\section{A.13 Proof of Proposition 9: Long-term yield}

Note that

$$
\nu_{1, t}+\nu_{2, t}=1
$$

Hence,

$$
\nu_{1, t}=1-\nu_{2, t}
$$

Because $\gamma_{1}<\gamma_{2}$, we have

$$
\nu_{1, t}^{\frac{\gamma_{1}}{\gamma_{2}}} \geq 1-\nu_{2, t}
$$

Also note that since

$$
\pi_{t}=\hat{\pi}_{k, t} \nu_{k, t}^{-\gamma_{k}}=\lambda_{k, 0} e^{-\hat{r}_{k} t} e^{-\frac{1}{2} \hat{\theta}_{k}^{2} t-\hat{\theta}_{k} Z_{t}} \nu_{k, t}^{-\gamma_{k}}
$$

we have

$$
\begin{aligned}
\pi_{t}^{-\frac{1}{\gamma_{k}}} & =\hat{\pi}_{k, t}^{-\frac{1}{\gamma_{k}}} \nu_{k, t} \\
\nu_{k, t} & =\pi_{t}^{-\frac{1}{\gamma_{k}}} \hat{\pi}_{k, t}^{\frac{1}{\gamma_{k}}} .
\end{aligned}
$$

Therefore,

$$
\left(\pi_{t}^{-\frac{1}{\gamma_{1}}} \hat{\pi}_{1, t}^{\frac{1}{\gamma_{1}}}\right)^{\frac{\gamma_{1}}{\gamma_{2}}} \geq 1-\lambda_{2,0}^{\frac{1}{\gamma_{2}}} \pi_{t}^{-\frac{1}{\gamma_{2}}} \hat{\pi}_{2, t}^{\frac{1}{\gamma_{2}}}
$$




$$
\begin{aligned}
\left(\pi_{t}^{-1} \hat{\pi}_{1, t}\right)^{\frac{1}{\gamma_{2}}} & \geq 1-\lambda_{2,0}^{\frac{1}{\gamma_{2}}} \pi_{t}^{-\frac{1}{\gamma_{2}}} \hat{\pi}_{2, t}^{\frac{1}{\gamma_{2}}} \\
\sum_{k=1}^{2} \hat{\pi}_{k, t}^{\frac{1}{\gamma_{2}}} & \geq \pi_{t}^{\frac{1}{\gamma_{2}}} \\
\left(\sum_{k=1}^{2} \hat{\pi}_{k, t}^{\frac{1}{\gamma_{2}}}\right)^{\gamma_{2}} & \geq \pi_{t}
\end{aligned}
$$

If we define $\bar{\gamma}_{2}=\max \left[1, \gamma_{2}\right]$, then

$$
\nu_{1, t}^{\frac{\gamma_{1}}{\bar{\gamma}_{2}}} \geq 1-\nu_{2, t}
$$

and so

$$
\left(\sum_{k=1}^{2} \hat{\pi}_{k, t}^{\frac{1}{\bar{\gamma}_{2}}}\right)^{\bar{\gamma}_{2}} \geq \pi_{t}
$$

Now note that

$$
\nu_{2, t}^{\frac{\gamma_{2}}{\gamma_{1}}} \leq 1-\nu_{1, t}
$$

Therefore,

$$
\begin{aligned}
\left(\pi_{t}^{-\frac{1}{\gamma_{2}}} \hat{\pi}_{2, t}^{\frac{1}{\gamma_{2}}}\right)^{\frac{\gamma_{2}}{\gamma_{1}}} & \leq 1-\hat{\pi}_{1, t}^{\frac{1}{\gamma_{1}}} \pi_{t}^{-\frac{1}{\gamma_{1}}} \\
\lambda_{2,0}^{\frac{1}{\gamma_{1}}} \pi_{t}^{-\frac{1}{\gamma_{1}}} \hat{\pi}_{2, t}^{\frac{1}{\gamma_{1}}} & \leq 1-\hat{\pi}_{1, t}^{\frac{1}{\gamma_{1}}} \pi_{t}^{-\frac{1}{\gamma_{1}}} \\
\pi_{t} & \geq\left(\sum_{k=1}^{2} \hat{\pi}_{k, t}^{\frac{1}{\gamma_{1}}}\right)^{\gamma_{1}}
\end{aligned}
$$

If we define $\underline{\gamma}_{1}=\min \left[1, \gamma_{1}\right]$, then

$$
\nu_{2, t}^{\frac{\gamma_{2}}{\gamma_{1}}} \leq 1-\nu_{1, t}
$$

Then,

$$
\pi_{t} \geq\left(\sum_{k=1}^{2} \hat{\pi}_{k, t}^{\frac{1}{\underline{\gamma}_{1}}}\right)^{\underline{\gamma}_{1}}
$$

Therefore,

$$
\left(\sum_{k=1}^{2} \hat{\pi}_{k, t}^{\frac{1}{\gamma_{2}}}\right)^{\gamma_{2}} \geq \pi_{t} \geq\left(\sum_{k=1}^{2} \hat{\pi}_{k, t}^{\frac{1}{\gamma_{1}}}\right)^{\gamma_{1}}
$$

and

$$
\left(\sum_{k=1}^{2} \hat{\pi}_{k, t}^{\frac{1}{\bar{\gamma}_{2}}}\right)^{\bar{\gamma}_{2}} \geq \pi_{t} \geq\left(\sum_{k=1}^{2} \hat{\pi}_{k, t}^{\frac{1}{\gamma_{1}}}\right)^{\underline{\gamma}_{1}} .
$$


The latter inequality implies that

$$
\left(\sum_{k=1}^{2} \hat{\pi}_{k, T}^{\frac{1}{\bar{\gamma}_{2}}}\right)^{\bar{\gamma}_{2}} \geq \pi_{T} \geq\left(\sum_{k=1}^{2} \hat{\pi}_{k, T}^{\frac{1}{\gamma_{1}}}\right)^{\underline{\gamma}_{1}}
$$

which implies that

$$
\left(\sum_{k=1}^{2}\left(\hat{\pi}_{k, T} \frac{X_{T}}{X_{0}}\right)^{\frac{1}{\bar{\gamma}_{2}}}\right)^{\bar{\gamma}_{2}} \geq \pi_{T} \frac{X_{T}}{X_{0}} \geq\left(\sum_{k=1}^{2}\left(\hat{\pi}_{k, T} \frac{X_{T}}{X_{0}}\right)^{\frac{1}{\underline{\gamma}_{1}}}\right)^{\underline{\gamma}_{1}}
$$

Since $f(x, y)=\left(x^{1 / \gamma}+y^{1 / \gamma}\right)^{\gamma}$ is strictly convex (concave) if and only if $\gamma<1(\gamma>1)$, it follows from Jensen's Inequality that

$$
\left(\sum_{k=1}^{2}\left(E_{0}\left[\hat{\pi}_{k, T} X_{T}\right]\right)^{\frac{1}{\bar{\gamma}_{2}}}\right)^{\bar{\gamma}_{2}} \geq E_{0}\left[\pi_{T} X_{T}\right] \geq\left(\sum_{k=1}^{2}\left(E_{0}\left[\hat{\pi}_{k, T} X_{T}\right]\right)^{\frac{1}{\underline{\gamma}_{1}}}\right)^{\underline{\gamma}_{1}}
$$

Recall that

$$
\frac{\hat{\pi}_{k, T}}{\hat{\pi}_{k, 0}} \frac{X_{T}}{X_{0}}=e^{-k_{k} T} \frac{M_{k, T}}{M_{k, 0}} e^{-\left(\gamma_{k}-\frac{1}{\psi_{k}}\right)\left(\omega_{T}-\omega_{0}\right)} .
$$

Wlog, $\hat{\pi}_{k, 0}=1$. Hence,

$$
\begin{aligned}
\hat{\pi}_{k, T} \frac{X_{T}}{X_{0}} & =e^{-k_{k} T} M_{k, T} e^{-\left(\gamma_{k}-\frac{1}{\psi_{k}}\right)\left(\omega_{T}-\omega_{0}\right)} \\
& =e^{-k_{k} T} M_{k, T} e^{-\left(\gamma_{k}-\frac{1}{\psi_{k}}\right)\left(\omega_{T}-\omega_{0}\right)},
\end{aligned}
$$

and so

$$
\begin{aligned}
E_{0}\left[\hat{\pi}_{k, T} \frac{X_{T}}{X_{0}}\right] & =e^{-k_{k} T} E_{0}\left[M_{k, T} e^{-\left(\gamma_{k}-\frac{1}{\psi_{k}}\right)\left(\omega_{T}-\omega_{0}\right)}\right] \\
& =e^{-k_{k} T} \hat{E}_{0}^{k}\left[e^{-\left(\gamma_{k}-\frac{1}{\psi_{k}}\right)\left(\omega_{T}-\omega_{0}\right)}\right] \\
& =e^{-k_{k} T} e^{\left(\gamma_{k}-\frac{1}{\psi_{k}}\right) \omega_{0}} \hat{E}_{0}^{k}\left[e^{-\left(\gamma_{k}-\frac{1}{\psi_{k}}\right) \omega_{T}}\right] \\
& =e^{-k_{k} T} \exp \left\{-\left(\gamma_{k}-\frac{1}{\psi_{k}}\right)\left(1-e^{-\lambda_{x} T}\right)\left(\hat{\omega}_{k}^{X}-\omega_{0}\right)+\frac{1}{2}\left(\gamma_{k}-\frac{1}{\psi_{k}}\right)^{2} \frac{1-e^{-2 \lambda_{x} T}}{2 \lambda_{x}} \sigma_{Y}^{2}\right\}
\end{aligned}
$$

It follows that

$$
\begin{aligned}
& \left(\sum_{k=1}^{2}\left(e^{-k_{k} T} \exp \left\{-\left(\gamma_{k}-\frac{1}{\psi_{k}}\right)\left(1-e^{-\lambda_{x} T}\right)\left(\hat{\omega}_{k}^{X}-\omega_{0}\right)+\frac{1}{2}\left(\gamma_{k}-\frac{1}{\psi_{k}}\right)^{2} \frac{1-e^{-2 \lambda_{x} T}}{2 \lambda_{x}} \sigma_{Y}^{2}\right\}\right)^{\frac{1}{\bar{\gamma}_{2}}}\right)^{\bar{\gamma}_{2}} \\
& \geq E_{t}\left[\pi_{T} X_{T}\right]
\end{aligned}
$$




$$
\geq\left(\sum_{k=1}^{2}\left(e^{-k_{k} T} \exp \left\{-\left(\gamma_{k}-\frac{1}{\psi_{k}}\right)\left(1-e^{-\lambda_{x} T}\right)\left(\hat{\omega}_{k}^{X}-\omega_{0}\right)+\frac{1}{2}\left(\gamma_{k}-\frac{1}{\psi_{k}}\right)^{2} \frac{1-e^{-2 \lambda_{x} T}}{2 \lambda_{x}} \sigma_{Y}^{2}\right\}\right)^{\frac{1}{\underline{\gamma}_{1}}}\right)^{\underline{\gamma}_{1}}
$$

which implies

$$
\begin{aligned}
& -\frac{1}{T} \ln \left[\left(\sum_{k=1}^{2}\left(e^{-k_{k} T} \exp \left\{-\left(\gamma_{k}-\frac{1}{\psi_{k}}\right)\left(1-e^{-\lambda_{x} T}\right)\left(\hat{\omega}_{k}^{X}-\omega_{0}\right)+\frac{1}{2}\left(\gamma_{k}-\frac{1}{\psi_{k}}\right)^{2} \frac{1-e^{-2 \lambda_{x} T}}{2 \lambda_{x}} \sigma_{Y}^{2}\right\}\right)^{\frac{1}{\underline{\gamma}_{1}}}\right)^{\underline{\gamma}_{1}}\right] \\
& \leq-\frac{1}{T} \ln v_{0, T}^{X} \\
& \leq-\frac{1}{T} \ln \left[\left(\sum_{k=1}^{2}\left(e^{-k_{k} T} \exp \left\{-\left(\gamma_{k}-\frac{1}{\psi_{k}}\right)\left(1-e^{-\lambda_{x} T}\right)\left(\hat{\omega}_{k}^{X}-\omega_{0}\right)+\frac{1}{2}\left(\gamma_{k}-\frac{1}{\psi_{k}}\right)^{2} \frac{1-e^{-2 \lambda_{x} T}}{2 \lambda_{x}} \sigma_{Y}^{2}\right\}\right)^{\frac{1}{\bar{\gamma}_{2}}}\right)^{\bar{\gamma}_{2}}\right] .
\end{aligned}
$$

Letting $T \rightarrow \infty$ gives

$$
\min \left(k_{1}, k_{2}\right) \leq-\lim _{T \rightarrow \infty} \frac{1}{T} \ln v_{0, T}^{X} \leq \min \left(k_{1}, k_{2}\right)
$$

and so

$$
-\lim _{T \rightarrow \infty} \frac{1}{T} \ln v_{0, T}^{X}=\min \left(k_{1}, k_{2}\right) .
$$

The other results in the proposition, for the yield on riskless bonds and the term premium, follow once we set $\sigma_{X}^{\text {sys }}=\mu_{X, i}=0$ in the equation above.

\section{A.14 Proof of Corollary 5: Survival and price impact under identical prefer- ences and different beliefs.}

The corollary follows immediately from Proposition 9 , after setting $\beta_{1}=\beta_{2}=\beta, \gamma_{1}=\gamma_{2}=\gamma$, $\psi_{1}=\psi_{2}=\psi$, and $\mu_{Y, 1}=\mu_{Y}$.

\section{A.15 Proof of Proposition 10: Risk premium and volatility of risky assets}

We shall derive results for a more general risky asset, which is a perpetual claim to the cash flow process, $X$, where the evolution of $X$ is given by

$$
\frac{d X_{t}}{X_{t}}=\mu_{X} d t+\sigma_{X}^{s y s} d Z_{t}+\sigma_{X}^{i d} d Z_{t}^{i d}
$$

where $Z_{t}^{i d}$ is a standard Brownian motion under $\mathbb{P}$, orthogonal to $Z_{t}$. Under probability measure $\mathbb{P}^{k}, k \in\{1,2\}$, the dynamics of the cash flow process are given by

$$
\frac{d X_{t}}{X_{t}}=\mu_{X, k} d t+\sigma_{X}^{s y s} d Z_{k, t}+\sigma_{X}^{i d} d Z_{t}^{i d},
$$


where $\mu_{X, k}$ is given by

$$
\frac{\mu_{X, k}-\mu_{X}}{\sigma_{X}^{s y s}}=\frac{\mu_{Y, k}-\mu_{Y}}{\sigma_{Y}}
$$

Then, to get the risk premium and the volatility of the stock market, we will set $\mu_{X}=\mu_{Y}$, $\sigma_{X}^{\text {sys }}=\sigma_{Y}$, and $\sigma_{X}^{i d}=0$.

The risk premium for the claim paying $X$ in perpetuity is given by the standard asset pricing equation:

$$
E_{t}\left[\frac{d P_{t}^{X}+X_{t} d t}{P_{t}^{X}}-r_{t} d t\right]=-E_{t}\left[\frac{d \pi_{t}}{\pi_{t}} \frac{d P_{t}^{X}}{P_{t}^{X}}\right] .
$$

Applying Ito's Lemma to $P_{t}^{X}=X_{t} p_{t}^{X}$ gives

$$
\begin{aligned}
\frac{d P_{t}^{X}}{P_{t}^{X}} & =\frac{d X_{t}}{X_{t}}+\frac{d p_{t}^{X}}{p_{t}^{X}}+\frac{d X_{t}}{X_{t}} \frac{d p_{t}^{X}}{p_{t}^{X}} \\
& =\mu_{X} d t+\sigma_{X}^{s y s} d Z_{t}+\sigma_{X}^{i d} d Z_{t}^{i d}+\frac{1}{p_{t}^{X}}\left[\frac{\partial p_{t}^{X}}{\partial \nu_{1, t}} \nu_{1, t}\left(\mu_{\nu_{1, t}} d t+\sigma_{\nu_{1, t}} d Z_{t}\right)+\frac{\partial p_{t}^{X}}{\partial \omega_{t}}\left(\lambda_{x}\left(\bar{\omega}-\omega_{t}\right) d t+\sigma_{Y} d Z_{t}\right)\right] \\
& +\frac{1}{2} \frac{1}{p_{t}^{X}} \frac{\partial^{2} p_{t}^{X}}{\partial \nu_{1, t}^{2}} \nu_{1, t}^{2} \sigma_{\nu_{1, t}}^{2} d t+\sigma_{X}^{s y s} \frac{1}{p_{t}^{X}} \frac{\partial p_{t}^{X}}{\partial \nu_{1, t}} \nu_{1, t} \sigma_{\nu_{1, t}} d t++\sigma_{X}^{s y s} \frac{1}{p_{t}^{X}} \frac{\partial p_{t}^{X}}{\partial \omega_{t}} \sigma_{Y} d t \\
& +\frac{1}{2} \frac{1}{p_{t}^{X}} \frac{\partial^{2} p_{t}^{X}}{\partial \omega_{t}^{2}} \sigma_{Y}^{2} d t .
\end{aligned}
$$

Thus, the total volatility of the return on the claim that pays $X$ in perpetuity, $\sigma_{R, t}^{X}$, is given by

$$
\sigma_{R, t}^{X}=\sqrt{\left(\sigma_{R, t}^{X, s y s}\right)^{2}+\left(\sigma_{R, t}^{X, i d}\right)^{2}}
$$

where the idiosyncratic component of the volatility of the claim's returns is given by

$$
\sigma_{R, t}^{X, i d}=\sigma_{X}^{i d}
$$

and the systematic component of the volatility of the claim's returns is given by

$$
\sigma_{R, t}^{X, s y s}=\sigma_{X}^{s y s}+\sigma_{\nu_{1}, t} \frac{\nu_{1, t}}{p_{t}^{X}} \frac{\partial p_{t}^{X}}{\partial \nu_{1, t}}+\sigma_{Y} \frac{1}{p_{t}^{X}} \frac{\partial p_{t}^{X}}{\partial \omega_{t}}
$$

Hence, substituting (??) into (A52) gives

$$
\mu_{R, t}^{X}-r_{t}=\theta_{t} \sigma_{R, t}^{X, s y s}
$$

where

$$
\mu_{R, t}^{X} d t=E_{t}\left[\frac{d P_{t}^{X}+X_{t} d t}{P_{t}^{X}}\right]
$$


Substituting (54) into (A53) gives

$$
\mu_{R, t}^{X}-r_{t}=\left(\mathbf{R}_{t} \sigma_{Y}+\left[\frac{\mu_{Y}-\boldsymbol{\mu}_{Y, t}}{\sigma_{Y}}\right]\right) \sigma_{R, t}^{X, s y s}
$$

Also, Agent $k$ 's perception of the risk premium for the claim paying $X$ in perpetuity is given by the standard asset pricing equation:

$$
E_{t}^{k}\left[\frac{d P_{t}^{X}+X_{t} d t}{P_{t}^{X}}-r_{t} d t\right]=-E_{t}^{k}\left[\frac{d \pi_{k, t}}{\pi_{k, t}} \frac{d P_{t}^{X}}{P_{t}^{X}}\right] .
$$

Hence,

$$
\mu_{R, k, t}^{X}-r_{t}=\theta_{k, t} \sigma_{R, t}^{X, s y s}
$$

where

$$
\mu_{R, k, t}^{X} d t=E_{t}^{k}\left[\frac{d P_{t}^{X}+X_{t} d t}{P_{t}^{X}}\right]
$$

Substituting (55) into (A54) gives

$$
\mu_{R, 1, t}^{X}-r_{t}=\left(\mathbf{R}_{t} \sigma_{Y}+\left[\frac{\mu_{Y}-\mu_{Y, 1}}{\sigma_{Y}}\right]\right) \sigma_{R, t}^{X, s y s}
$$

Agent 2's perception of the risk premium is given by

$$
\mu_{R, 2, t}^{X}-r_{t}=\left(\mathbf{R}_{t} \sigma_{Y}+\left[\frac{\mu_{Y}-\mu_{Y, 2}}{\sigma_{Y}}\right]\right) \sigma_{R, t}^{X, s y s},
$$

Setting $\mu_{X}=\mu_{Y}, \sigma_{X}^{\text {sys }}=\sigma_{Y}$, and $\sigma_{X}^{i d}=0$ in the above expressions gives the results in the proposition.

\section{A.16 Proof of Proposition 11}

$$
\begin{aligned}
\phi_{n, 1, t, u} & =E_{t}\left[\left(\frac{\hat{\pi}_{1, u}}{\hat{\pi}_{1, t}}\right)^{1-\frac{n}{\gamma_{1}}}\left(\frac{\hat{\pi}_{2, u}}{\hat{\pi}_{2, t}}\right)^{\frac{n}{\gamma_{2}}} \frac{X_{u}}{X_{t}} 1_{\left\{\frac{\hat{\pi}_{1, u}}{\hat{\pi}_{2, u}}>R\right\}}\right] \\
& =E_{t}\left[e^{\left(1-\frac{n}{\gamma_{1}}\right)\left(\varsigma_{1, u}-\varsigma_{1, t}\right)+\frac{n}{\gamma_{2}}\left(\varsigma_{2, u}-\varsigma_{2, t}\right)+x_{u}-x_{t}} 1_{\left\{\varsigma_{1, u}-\varsigma_{2, u}>\rho\right\}}\right] .
\end{aligned}
$$

Taking the Fourier transform of the above expression yields

$$
\begin{aligned}
\mathcal{F}\left[\phi_{n, 1, t, u}, z\right] & =E_{t}\left[e^{\left(1-\frac{n}{\gamma_{1}}\right)\left(\varsigma_{1, u}-\varsigma_{1, t}\right)+\frac{n}{\gamma_{2}}\left(\varsigma_{2, u}-\varsigma_{2, t}\right)+x_{u}-x_{t}} \mathcal{F}\left[1_{\left\{\varsigma_{1, u}-\varsigma_{2, u}>\rho\right\}}, x\right]\right] \\
& =E_{t}\left[e^{\left(1-\frac{n}{\gamma_{1}}\right)\left(\varsigma_{1, u}-\varsigma_{1, t}\right)+\frac{n}{\gamma_{2}}\left(\varsigma_{2, u}-\varsigma_{2, t}\right)+x_{u}-x_{t}}\left(\frac{e^{i z\left(\varsigma_{1, u}-\varsigma_{2, u}\right)}}{i z}+\pi \delta(z)\right)\right] \\
& =\frac{1}{i z} E_{t}\left[e^{\left(1-\frac{n}{\gamma_{1}}\right)\left(\varsigma_{1, u}-\varsigma_{1, t}\right)+\frac{n}{\gamma_{2}}\left(\varsigma_{2, u}-\varsigma_{2, t}\right)+i z\left(\varsigma_{1, u}-\varsigma_{2, u}\right)+x_{u}-x_{t}}\right]
\end{aligned}
$$




$$
\begin{aligned}
& +\pi \delta(z) E_{t}\left[e^{\left(1-\frac{n}{\gamma_{1}}\right)\left(\varsigma_{1, u}-\varsigma_{1, t}\right)+\frac{n}{\gamma_{2}}\left(\varsigma_{2, u}-\varsigma_{2, t}\right)+x_{u}-x_{t}}\right] \\
& =e^{-\left[\left(1-\frac{n}{\gamma_{1}}\right) \varsigma_{1, t}+\frac{n}{\gamma_{2}} \varsigma_{2, t}\right]-x_{t}}\left(\frac{1}{i z} E_{t}\left[e^{\left(1-\frac{n}{\gamma_{1}}\right) \varsigma_{1, u}+\frac{n}{\gamma_{2}} \varsigma_{2, u}+i z\left(\varsigma_{1, u}-\varsigma_{2, u}\right)+x_{u}}\right]\right. \\
& \left.+\pi \delta(z) E_{t}\left[e^{\left(1-\frac{n}{\gamma_{1}}\right) \varsigma_{1, u}+\frac{n}{\gamma_{2}} \varsigma_{2, u}+x_{u}}\right]\right) \\
& =e^{-\left[\left(1-\frac{n}{\gamma_{1}}\right) \varsigma_{1, t}+\frac{n}{\gamma_{2}} \varsigma_{2, t}\right]-x_{t}}\left(\frac{1}{i z} \mathcal{M}\left(\varsigma_{1, t}, \varsigma_{2, t}, x_{t}, u-t,\left(1-\frac{n}{\gamma_{1}}\right)+i z, \frac{n}{\gamma_{2}}-i z, 1\right)\right. \\
& \left.+\pi \delta(z) \mathcal{M}\left(\varsigma_{1, t}, \varsigma_{2, t}, x_{t}, u-t, 1-\frac{n}{\gamma_{1}}, \frac{n}{\gamma_{2}}, 1\right)\right) .
\end{aligned}
$$

Taking the inverse Fourier transform gives

$$
\begin{aligned}
\phi_{n, 1, t, u} & =e^{-\left[\left(1-\frac{n}{\gamma_{1}}\right) \varsigma_{1, t}+\frac{n}{\gamma_{2}} \varsigma_{2, t}\right]-x_{t}}\left[\frac{1}{2 \pi} \int_{-\infty}^{\infty} e^{i \rho z} \frac{1}{i z} \mathcal{M}\left(\varsigma_{1, t}, \varsigma_{2, t}, x_{t}, u-t,\left(1-\frac{n}{\gamma_{1}}\right)+i z, \frac{n}{\gamma_{2}}-i z, 1\right) d z\right. \\
& \left.+\pi \frac{1}{2 \pi} \int_{-\infty}^{\infty} e^{i \rho z} \delta(z) d z \mathcal{M}\left(\varsigma_{1, t}, \varsigma_{2, t}, x_{t}, u-t, 1-\frac{n}{\gamma_{1}}, \frac{n}{\gamma_{2}}, 1\right)\right] \\
& =e^{-\left[\left(1-\frac{n}{\gamma_{1}}\right) \varsigma_{1, t}+\frac{n}{\gamma_{2}} \varsigma_{2, t}\right]-x_{t}}\left[\frac{1}{2 \pi} \int_{-\infty}^{\infty} e^{i \rho z} \frac{1}{i z} \mathcal{M}\left(\varsigma_{1, t}, \varsigma_{2, t}, x_{t}, u-t,\left(1-\frac{n}{\gamma_{1}}\right)+i z, \frac{n}{\gamma_{2}}-i z, 1\right) d z\right. \\
& \left.+\pi \frac{1}{2 \pi} \int_{-\infty}^{\infty} e^{i \rho z} \delta(z) d z \mathcal{M}\left(\varsigma_{1, t}, \varsigma_{2, t}, x_{t}, u-t, 1-\frac{n}{\gamma_{1}}, \frac{n}{\gamma_{2}}, 1\right)\right] \\
& =e^{-\left[\left(1-\frac{n}{\gamma_{1}}\right) \varsigma_{1, t}+\frac{n}{\gamma_{2}} \varsigma_{2, t}\right]-x_{t}}\left[\frac{1}{2 \pi} \int_{-\infty}^{\infty} e^{i \rho z} \frac{1}{i z} \mathcal{M}\left(\varsigma_{1, t}, \varsigma_{2, t}, x_{t}, u-t,\left(1-\frac{n}{\gamma_{1}}\right)+i z, \frac{n}{\gamma_{2}}-i z, 1\right) d z\right. \\
& \left.+\frac{1}{2} \mathcal{M}\left(\varsigma_{1, t}, \varsigma_{2, t}, x_{t}, u-t, 1-\frac{n}{\gamma_{1}}, \frac{n}{\gamma_{2}}, 1\right)\right],
\end{aligned}
$$

and so we obtain

$$
\begin{aligned}
\phi_{n, 1, t, u} & =e^{-\left[\left(1-\frac{n}{\gamma_{1}}\right) \varsigma_{1, t}+\frac{n}{\gamma_{2}} \varsigma_{2, t}\right]-x_{t}}\left[\frac{1}{2 \pi} \int_{-\infty}^{\infty} e^{i \rho z} \frac{1}{i z} \mathcal{M}\left(\varsigma_{1, t}, \varsigma_{2, t}, x_{t}, u-t,\left(1-\frac{n}{\gamma_{1}}\right)+i z, \frac{n}{\gamma_{2}}-i z, 1\right) d z\right. \\
& \left.+\frac{1}{2} \mathcal{M}\left(\varsigma_{1, t, \varsigma_{2}, t}, x_{t}, u-t, 1-\frac{n}{\gamma_{1}}, \frac{n}{\gamma_{2}}, 1\right)\right]
\end{aligned}
$$

Now observe that

$$
\begin{aligned}
& \int_{-\infty}^{\infty} e^{i \rho z} \frac{1}{i z} \mathcal{M}\left(\varsigma_{1, t}, \varsigma_{2, t}, x_{t}, u-t,\left(1-\frac{n}{\gamma_{1}}\right)+i z, \frac{n}{\gamma_{2}}-i z, 1\right) d z \\
& =\int_{-\infty}^{0} e^{i \rho z} \frac{1}{i z} \mathcal{M}\left(\varsigma_{1, t}, \varsigma_{2, t}, x_{t}, u-t,\left(1-\frac{n}{\gamma_{1}}\right)+i z, \frac{n}{\gamma_{2}}-i z, 1\right) d z \\
& +\int_{0}^{\infty} e^{i \rho z} \frac{1}{i z} \mathcal{M}\left(\varsigma_{1, t}, \varsigma_{2, t}, x_{t}, u-t,\left(1-\frac{n}{\gamma_{1}}\right)+i z, \frac{n}{\gamma_{2}}-i z, 1\right) d z
\end{aligned}
$$




$$
\begin{aligned}
& =\int_{0}^{\infty} e^{-i \rho z} \frac{1}{-i z} \mathcal{M}\left(\varsigma_{1, t}, \varsigma_{2, t}, x_{t}, u-t,\left(1-\frac{n}{\gamma_{1}}\right)-i z, \frac{n}{\gamma_{2}}+i z, 1\right) d z \\
& +\int_{0}^{\infty} e^{i \rho z} \frac{1}{i z} \mathcal{M}\left(\varsigma_{1, t}, \varsigma_{2, t}, x_{t}, u-t,\left(1-\frac{n}{\gamma_{1}}\right)+i z, \frac{n}{\gamma_{2}}-i z, 1\right) d z
\end{aligned}
$$

If $\left(\varsigma_{1, t}, \varsigma_{2, t}, x_{t}\right)$ is analytic affine, then $\mathcal{M}\left(\varsigma_{1, t}, \varsigma_{2, t}, x_{t}, u-t, z_{1}, z_{2}, z_{3}\right), z_{1}, z_{2}, z_{3} \in \mathbb{C}$ is a holomorphic function whose restriction to the real numbers is real-valued, and so

$$
\overline{\mathcal{M}}\left(\varsigma_{1, t}, \varsigma_{2, t}, x_{t}, u-t, z_{1}, z_{2}, z_{3}\right)=\mathcal{M}\left(\varsigma_{1, t}, \varsigma_{2, t}, x_{t}, u-t, \bar{z}_{1}, \bar{z}_{2}, \bar{z}_{3}\right)
$$

where $\bar{z}$ denotes the complex conjugate. Therefore

$$
\mathcal{M}\left(\varsigma_{1, t}, \varsigma_{2, t}, x_{t}, u-t,\left(1-\frac{n}{\gamma_{1}}\right)-i z, \frac{n}{\gamma_{2}}+i z, 1\right)=\overline{\mathcal{M}}\left(\varsigma_{1, t}, \varsigma_{2, t}, x_{t}, u-t,\left(1-\frac{n}{\gamma_{1}}\right)+i z, \frac{n}{\gamma_{2}}-i z, 1\right)
$$

and so

$$
\begin{aligned}
& \int_{-\infty}^{\infty} e^{i \rho z} \frac{1}{i z} \mathcal{M}\left(\varsigma_{1, t}, \varsigma_{2, t}, x_{t}, u-t,\left(1-\frac{n}{\gamma_{1}}\right)+i z, \frac{n}{\gamma_{2}}-i z, 1\right) d z \\
& =\int_{0}^{\infty} \frac{e^{i \rho z} \frac{1}{i z} \mathcal{M}\left(\varsigma_{1, t}, \varsigma_{2, t}, x_{t}, u-t,\left(1-\frac{n}{\gamma_{1}}\right)+i z, \frac{n}{\gamma_{2}}-i z, 1\right)}{d z} \\
& +\int_{0}^{\infty} e^{i \rho z} \frac{1}{i z} \mathcal{M}\left(\varsigma_{1, t}, \varsigma_{2, t}, x_{t}, u-t,\left(1-\frac{n}{\gamma_{1}}\right)+i z, \frac{n}{\gamma_{2}}-i z, 1\right) d z \\
& =2 \int_{0}^{\infty} \operatorname{Re}\left[e^{i \rho z} \frac{1}{i z} \mathcal{M}\left(\varsigma_{1, t}, \varsigma_{2, t}, x_{t}, u-t,\left(1-\frac{n}{\gamma_{1}}\right)+i z, \frac{n}{\gamma_{2}}-i z, 1\right)\right] d z \\
& =2 \int_{0}^{\infty} \frac{1}{z} \operatorname{Im}\left[e^{i \rho z} \mathcal{M}\left(\varsigma_{1, t}, \varsigma_{2, t}, x_{t}, u-t,\left(1-\frac{n}{\gamma_{1}}\right)+i z, \frac{n}{\gamma_{2}}-i z, 1\right)\right] d z
\end{aligned}
$$

Therefore, we obtain (70). Recall that

$$
\phi_{n, 2, t, u}=E_{t}\left[\left(\frac{\hat{\pi}_{1, u}}{\hat{\pi}_{1, t}}\right)^{\frac{n}{\gamma_{1}}}\left(\frac{\hat{\pi}_{2, u}}{\hat{\pi}_{2, t}}\right)^{1-\frac{n}{\gamma_{2}}} \frac{X_{u}}{X_{t}} 1_{\left\{\frac{\hat{\pi}_{1, u}}{\hat{\pi}_{2, u}}<R\right\}}\right] .
$$

Hence

$$
\phi_{n, 2, t, u}=E_{t}\left[\left(\frac{\hat{\pi}_{1, u}}{\hat{\pi}_{1, t}}\right)^{\frac{n}{\gamma_{1}}}\left(\frac{\hat{\pi}_{2, u}}{\hat{\pi}_{2, t}}\right)^{1-\frac{n}{\gamma_{2}}} \frac{X_{u}}{X_{t}}\right]-E_{t}\left[\left(\frac{\hat{\pi}_{1, u}}{\hat{\pi}_{1, t}}\right)^{\frac{n}{\gamma_{1}}}\left(\frac{\hat{\pi}_{2, u}}{\hat{\pi}_{2, t}}\right)^{1-\frac{n}{\gamma_{2}}} \frac{X_{u}}{X_{t}} 1_{\left\{\frac{\hat{\pi}_{1, u}}{\hat{\pi}_{2, u}}>R\right\}}\right] .
$$

Following the same steps as the derivation of (A55), we obtain

$$
\begin{aligned}
\phi_{n, 2, t, u} & =e^{-\left[\frac{n}{\gamma_{1}} \varsigma_{1, t}+\left(1-\frac{n}{\gamma_{2}}\right) \varsigma_{2, t}\right]-x_{t}}\left[-\frac{1}{2 \pi} \int_{-\infty}^{\infty} e^{i \rho z} \frac{1}{i z} \mathcal{M}\left(\varsigma_{1, t}, \varsigma_{2, t}, x_{t}, u-t, \frac{n}{\gamma_{1}}+i z, 1-\frac{n}{\gamma_{2}}-i z, 1\right) d z\right. \\
& \left.+\frac{1}{2} \mathcal{M}\left(\varsigma_{1, t}, \varsigma_{2, t}, x_{t}, u-t, \frac{n}{\gamma_{1}}, 1-\frac{n}{\gamma_{2}}, 1\right)\right] .
\end{aligned}
$$

If $\left(\varsigma_{1, t}, \varsigma_{2, t}, x_{t}\right)$ is analytic affine, then we obtain $(71)$. 


\section{A.17 The Distribution of The Consumption Share}

In this section we give the conditional probability density function of the consumption share $\nu_{1}$, and derive its long-run behavior.

Proposition A1 (Density function for the consumption share) The density function for $\nu_{1, t+u}$, conditional on $q_{t}$ and $\omega_{t}$ is denoted by $p_{\nu_{1, t+u}}\left(v \mid q_{t}, \omega_{t}\right)$, and is given by

$$
\begin{aligned}
& p_{\nu_{1, t+u}}\left(v \mid q_{t}, \omega_{t}\right)= \\
& \frac{1}{\sqrt{\sigma_{q}^{2} u+2 d_{\omega} \frac{1-e^{-\lambda_{x} u}}{\lambda_{x}} \sigma_{q} \sigma_{Y}+d_{\omega}^{2} \frac{1-e^{-2 \lambda_{x} u}}{2 \lambda_{x}} \sigma_{Y}^{2}}} \phi\left(\frac{\ln \frac{h_{1}(v)}{h_{1}\left(\nu_{1, t}\right)}-\left[\mu_{q} u+d_{\omega}\left(1-e^{-\lambda_{x} u}\right)\left(\bar{\omega}-\omega_{t}\right)\right]}{\sqrt{\sigma_{q}^{2} u+2 d_{\omega} \frac{1-e^{-\lambda_{x} u}}{\lambda_{x}} \sigma_{q} \sigma_{Y}+d_{\omega}^{2} \frac{1-e^{-2 \lambda_{x} u}}{2 \lambda_{x}} \sigma_{Y}^{2}}}\right) \frac{h_{1}^{\prime}(v)}{h_{1}(v)},
\end{aligned}
$$

where $\phi(z)=\frac{1}{\sqrt{2 \pi}} e^{-\frac{1}{2} z^{2}}$ is the standard normal density function and

$$
\begin{aligned}
\mu_{q} & =\left(\beta_{2}-\beta_{1}\right)+\frac{1}{2}\left(\sigma_{\xi, 2}^{2}-\sigma_{\xi, 1}^{2}\right)+\left(\frac{1}{\psi_{2}}-\frac{1}{\psi_{1}}\right)\left(\mu_{Y}-\frac{1}{2} \sigma_{Y}^{2}\right), \\
\sigma_{q} & =\sigma_{\xi, 1}-\sigma_{\xi, 2}+\left(\frac{1}{\psi_{2}}-\frac{1}{\psi_{1}}\right) \sigma_{Y} \\
d_{\omega} & =\left(\gamma_{2}-\frac{1}{\psi_{2}}\right)-\left(\gamma_{1}-\frac{1}{\psi_{1}}\right) \\
h_{1}(v) & =v^{\gamma_{1}}(1-v)^{-\gamma_{2}} \\
\mathbf{R}_{t}(v) & =\left(v \frac{1}{\gamma_{1}}+(1-v) \frac{1}{\gamma_{2}}\right)^{-1} .
\end{aligned}
$$

If both agents have the same survivial indices, that is, $\mu_{q}=0$, then

$$
\lim _{u \rightarrow \infty} p_{\nu_{1, t+u}}\left(v \mid \nu_{1, t}\right)=\frac{1}{2}(\delta(v)+\delta(v-1))
$$

where $\delta(\cdot)$ is the Dirac-delta function.

\section{Proof of Proposition A1}

Note that

$$
e^{\Delta_{t}}=h_{1}\left(\nu_{1, t}\right)
$$

The cumulative distribution function for $\nu_{1, t+u}$, conditional on $\nu_{t}$ is given by

$$
\begin{aligned}
& \operatorname{Pr}\left(\nu_{1, t+u} \leq v \mid q_{t}, \omega_{t}\right)=\operatorname{Pr}\left(h_{1}^{-1}\left(e^{\Delta_{t}}\right) \leq v \mid q_{t}, \omega_{t}\right) \\
= & \operatorname{Pr}\left(e^{\Delta_{t}} \leq h_{1}(v) \mid q_{t}, \omega_{t}\right) .
\end{aligned}
$$


The previous line shows that we shall not need to compute the inverse function $h_{1}^{-1}(\cdot)$. Coupled with the fact that $\Delta$ is Gaussian, this means deriving the cumulative distribution function is straightforward:

$$
\begin{aligned}
\operatorname{Pr}\left(e^{\Delta_{t+u}} \leq h_{1}(v) \mid q_{t}, \omega_{t}\right) & =\operatorname{Pr}\left(\Delta_{t+u} \leq \ln h_{1}(v) \mid q_{t}, \omega_{t}\right) \\
& =\operatorname{Pr}\left(q_{t+u}+d_{\omega} \omega_{t+u} \leq \ln h_{1}(v) \mid q_{t}, \omega_{t}\right) .
\end{aligned}
$$

Now observe that

$$
\begin{aligned}
\operatorname{Pr}\left(q_{t+u}+d_{\omega} \omega_{t+u} \leq \ln h_{1}(v) \mid q_{t}, \omega_{t}\right) & =E_{t}\left[1_{\left\{q_{t+u}+d_{\omega} \omega_{t+u} \leq \ln h_{1}(v)\right\}}\right] \\
& =E_{t}\left[1_{\left\{q_{t+u}+d_{\omega} \omega_{t+u} \leq \ln h_{1}(v)\right\}}\right] \\
& =1-E_{t}\left[1_{\left\{q_{t+u}+d_{\omega} \omega_{t+u}>\ln h_{1}(v)\right\}}\right] .
\end{aligned}
$$

Lemma A3 implies that

$$
\begin{aligned}
& E_{t}\left[1_{\left\{q_{t+u}+d_{\omega} \omega_{t+u}>\ln h_{1}(v)\right\}}\right]=\Phi\left(\frac{q_{t}+\mu_{q} u+d_{\omega}\left[e^{-\lambda_{x} u} \omega_{t}+\left(1-e^{-\lambda_{x} u}\right) \bar{\omega}\right]-\ln h_{1}(v)}{\sqrt{\sigma_{q}^{2} u+2 d_{\omega} \frac{1-e^{-\lambda_{x} u}}{\lambda_{x}} \sigma_{q} \sigma_{Y}+d_{\omega}^{2} \frac{1-e^{-2 \lambda_{x} u}}{2 \lambda_{x}} \sigma_{Y}^{2}}}\right) \\
& =\Phi\left(\frac{q_{t}+d_{\omega} \omega_{t}+\mu_{q} u+d_{\omega}\left(1-e^{-\lambda_{x} u}\right)\left(\bar{\omega}-\omega_{t}\right)-\ln h_{1}(v)}{\sqrt{\sigma_{q}^{2} u+2 d_{\omega} \frac{1-e^{-\lambda_{x} u}}{\lambda_{x}}} \sigma_{q} \sigma_{Y}+d_{\omega}^{2} \frac{1-e^{-2 \lambda_{x} u}}{2 \lambda_{x}} \sigma_{Y}^{2}}\right)
\end{aligned}
$$

where

$$
\begin{aligned}
& \mu_{q}=\left(\beta_{2}-\beta_{1}\right)+\frac{1}{2}\left(\sigma_{\xi, 2}^{2}-\sigma_{\xi, 1}^{2}\right)+\left(\frac{1}{\psi_{2}}-\frac{1}{\psi_{1}}\right)\left(\mu_{Y}-\frac{1}{2} \sigma_{Y}^{2}\right) \\
& \sigma_{q}=\sigma_{\xi, 1}-\sigma_{\xi, 2}+\left(\frac{1}{\psi_{2}}-\frac{1}{\psi_{1}}\right) \sigma_{Y} \\
& d_{\omega}=\left(\gamma_{2}-\frac{1}{\psi_{2}}\right)-\left(\gamma_{1}-\frac{1}{\psi_{1}}\right) .
\end{aligned}
$$

Hence

$$
\operatorname{Pr}\left(\nu_{1, t+u} \leq v \mid q_{t}, \omega_{t}\right)=\Phi\left(\frac{\ln h_{1}(v)-\left[q_{t}+d_{\omega} \omega_{t}+\mu_{q} u+d_{\omega}\left(1-e^{-\lambda_{x} u}\right)\left(\bar{\omega}-\omega_{t}\right)\right]}{\sqrt{\sigma_{q}^{2} u+2 d_{\omega} \frac{1-e^{-\lambda_{x} u}}{\lambda_{x}} \sigma_{q} \sigma_{Y}+d_{\omega}^{2} \frac{1-e^{-2 \lambda_{x} u}}{2 \lambda_{x}} \sigma_{Y}^{2}}}\right)
$$

Since $q_{t}+d_{\omega} \omega_{t}=\ln h_{1}\left(\nu_{1, t}\right)$, we have

$$
\operatorname{Pr}\left(\nu_{1, t+u} \leq v \mid q_{t}, \omega_{t}\right)=\Phi\left(\frac{\ln \frac{h_{1}(v)}{h_{1}\left(\nu_{1, t}\right)}-\left[\mu_{q} u+d_{\omega}\left(1-e^{-\lambda_{x} u}\right)\left(\bar{\omega}-\omega_{t}\right)\right]}{\sqrt{\sigma_{q}^{2} u+2 d_{\omega} \frac{1-e^{-\lambda_{x} u}}{\lambda_{x}}} \sigma_{q} \sigma_{Y}+d_{\omega}^{2} \frac{1-e^{-2 \lambda_{x} u}}{2 \lambda_{x}} \sigma_{Y}^{2}}\right)
$$

The density function $p_{\nu_{1, t+u}}\left(v \mid q_{t}, \omega_{t}\right)$ is given by

$p_{\nu_{1, t+u}}\left(v \mid q_{t}, \omega_{t}\right)$ 


$$
\begin{aligned}
& =\frac{d \operatorname{Pr}\left(\nu_{1, t+u} \leq v \mid q_{t}, \omega_{t}\right)}{d v} \\
& =\frac{1}{\sqrt{\sigma_{q}^{2} u+2 d_{\omega} \frac{1-e^{-\lambda_{x} u}}{\lambda_{x}} \sigma_{q} \sigma_{Y}+d_{\omega}^{2} \frac{1-e^{-2 \lambda_{x} u}}{2 \lambda_{x}} \sigma_{Y}^{2}}} \phi\left(\frac{\ln \frac{h_{1}(v)}{h_{1}\left(\nu_{1, t}\right)}-\left[\mu_{q} u+d_{\omega}\left(1-e^{-\lambda_{x} u}\right)\left(\bar{\omega}-\omega_{t}\right)\right]}{\sqrt{\sigma_{q}^{2} u+2 d_{\omega} \frac{1-e^{-\lambda_{x} u}}{\lambda_{x}} \sigma_{q} \sigma_{Y}+d_{\omega}^{2} \frac{1-e^{-2 \lambda_{x} u}}{2 \lambda_{x}} \sigma_{Y}^{2}}}\right) \frac{h_{1}^{\prime}(v)}{h_{1}(v)}
\end{aligned}
$$

Since

$$
\frac{h_{1}^{\prime}(v)}{h_{1}(v)}=\frac{\gamma_{1} \gamma_{2}}{v(1-v) \mathbf{R}_{t}(v)},
$$

we obtain (A56). When $\mu_{q}=0$, the limit of (A56) as $u \rightarrow \infty$ when $v \in(0,1)$ gives zero. When $v=0$ or 1 , the limit is infinite, but symmetry and the fact that $p_{\nu_{1, t+u}}\left(v \mid q_{t}, \omega_{t}\right)$ is a probability density function (and hence integrates to one) implies that $\lim _{u \rightarrow \infty} p_{\nu_{1, t+u}}\left(v=0 \mid q_{t}, \omega_{t}\right)=\frac{1}{2} \delta(v)$ and $\lim _{u \rightarrow \infty} p_{\nu_{1, t+u}}\left(v=1 \mid q_{t}, \omega_{t}\right)=\frac{1}{2} \delta(v-1)$. 


\section{References}

Abadir, K. M., 1999, "An Introduction to Hypergeometric Functions for Economists," Econometric Reviews, 18, 287-330.

Abel, A. B., 1990, "Asset Prices Under Habit Formation and Catching Up with the Joneses," American Economic Review, 80, 38-42.

Abel, A. B., 1999, "Risk Premia and Term Premia in General Equilibrium," Journal of Monetary Economics, 43, 3-33.

Basak, S., 2000, "A Model of Dynamic Equilibrium Asset Pricing with Extraneous Risk," Journal of Economic Dynamics and Control, 24, 63-95.

Basak, S., 2005, "Asset Pricing with Heterogeneous Beliefs," Journal of Banking and Finance, 29, $2849-2881$.

Basak, S., and D. Cuoco, 1998, "An Equilibrium Model with Restricted Stock Market Participation," Review of Financial Studies, 11, 309-341.

Beber, A., A. Buraschi, and F. Breedon, 2009, "Differences in Beliefs and Currency Risk Premia," forthcoming in Journal of Financial Economics.

Benninga, S., and J. Mayshar, 2000, "Heterogeneity and Option Pricing," Review of Derivatives Research, 4, 7-27.

Berrada, T., 2006, "Incomplete Information, Heterogeneity and Asset Pricing," Journal of Financial Econometrics, 4, 136-160.

Berrada, T., and J. Hugonnier, 2011, "Incomplete Information, Idiosyncratic Volatility and Stock Returns," Working Paper, University of Geneva and Swiss Finance Institute.

Bhamra, H. S., and R. Uppal, 2009, "The Effect of Introducing a Non-Redundant Derivative on the Volatility of Stock-Market Returns when Agents Differ in Risk Aversion," Review of Financial Studies, 22, 2303-2330.

Borovička, J., 2012, "Survival and Long-Run Dynamics with Heterogeneous Beliefs Under Recursive Preferences," Working Paper, University of Chicago.

Buraschi, A., and A. Jiltsov, 2006, "Model Uncertainty and Option Markets with Heterogeneous Agents," Journal of Finance, 61, 2841-2897.

Buraschi, A., F. Trojani, and A. Vedolin, 2009, "When Uncertainty Blows in the Orchard: Comovement and Equilibrium Volatility Risk Premia," Working Paper, Imperial College.

Buraschi, A., F. Trojani, and A. Vedolin, 2010, "Economic Uncertainty, Disagreement, and Credit Markets," Working Paper, Imperial College.

Calin, O. L., Y. Chen, T. F. Cosimano, and A. A. Himonas, 2005, "Solving Asset Pricing Models when the Price-Dividend Function is Analytic," Econometrica, 73, 961-982.

Campbell, J. Y., and J. H. Cochrane, 1999, "By Force of Habit: A Consumption-Based Explanation of Aggregate Stock Market Behavior," Journal of Political Economy, 107, 205-251.

Cecchetti, S. G., P.-s. Lam, and N. C. Mark, 2000, "Asset Pricing with Distorted Beliefs: Are Equity Returns Too Good to Be True?," American Economic Review, 90, 787-805. 
Chan, Y. L., and L. Kogan, 2002, "Catching Up with the Joneses: Heterogeneous Preferences and the Dynamics of Asset Prices," Journal of Political Economy, 110, 1255-1285.

Cochrane, J. H., 2001, Asset Pricing, Princeton University Press.

Constantinides, G. M., 1990, "Habit Formation: A Resolution of the Equity Premium Puzzle," Journal of Political Economy, 98, 519-543.

Cvitanić, J., E. Jouini, S. Malamud, and C. Napp, 2012, "Financial Market Equilibrium with Heterogeneous Agents," Review of Finance, 16, 285-321.

Cvitanić, J., and S. Malamud, 2009a, "Equilibrium Driven by Discounted Dividend Volatility," Working Paper.

Cvitanić, J., and S. Malamud, 2009b, "Relative Extinction of Heterogeneous Agents," Working Paper.

Cvitanić, J., and S. Malamud, 2009c, "Risk Aversion and Equilibrium Optimal Portfolios in Large Markets," Working Paper.

David, A., 2008, "Heterogenous Beliefs, Speculation, and the Equity Premium," Journal of Finance, $63,41-83$.

David, A., and P. Veronesi, 2002, "Option Prices with Uncertain Fundamentals: Theory and Evidence on the Dynamics of Implied Volatilities," Working Paper, University of Chicago.

Duffie, D., 2001, Dynamic Asset Pricing Theory, Princeton University Press, Princeton, 3rd edn.

Duffie, D., N. Garleânu, and L. H. Pedersen, 2002, "Securities Lending, Shorting, and Pricing," Journal of Financial Economics, 66, 307-339.

Duffie, J. D., J. Pan, and K. Singleton, 2000, "Transform Analysis and Asset Pricing for Affine Jump-Diffusions," Econometrica, 68, 1343-1376.

Dumas, B., 1989, "Two-Person Dynamic Equilibrium in the Capital Market," Review of Financial Studies, 2, 157-188.

Dumas, B., A. Kurshev, and R. Uppal, 2009, "Equilibrium Portfolio Strategies in the Presence of Sentiment Risk and Excess Volatility," Journal of Finance, 64, 579-629.

Epstein, L. G., and S. Zin, 1989, "Substitution, Risk Aversion and the Temporal Behavior of Consumption and Asset Returns: A Theoretical Framework," Econometrica, 57, 937-969.

Fedyk, Y., C. Heyerdahl-Larsen, and J. Walden, 2012, "Market Selection and Welfare in a MultiAsset Economy," Working Paper, London Business School.

Friedlander, F. G., and M. S. Joshi, 1999, Introduction to the Theory of Distributions, Cambridge University Press.

Gallmeyer, M., 2000, "Beliefs and Volatility," Working Paper, Carnegie Mellon University.

Gallmeyer, M., and B. Hollifield, 2008, "An Examination of Heterogeneous Beliefs with a Short Sale Constraint," Review of Finance, 12, 323-364.

Garlappi, L., and G. Skoulakis, 2011, "Taylor Series Approximations to Expected Utility and Optimal Portfolio Choice," Mathematics and Financial Economics, , 5, 121-156. 
Garleânu, N., and S. Panageas, 2008, "Young, Old, Conservative and Bold: The Implications of Heterogeneity and Finite Lives for Asset Pricing," Working Paper, University of California at Berkeley and University of Chicago.

Gollier, C., and R. Zeckhauser, 2005, "Aggregation of Heterogeneous Time Preferences," Journal of Political Economy, 113, 878-896.

Gomes, F., and A. Michaelides, 2008, "Asset Pricing with Limited Risk Sharing and Heterogeneous Agents," Review of Financial Economics, 21, 415-448.

Grossman, S. J., and J. E. Stiglitz, 1980, "On the Impossibility of Informationally Efficient Markets," American Economic Review, 70, 393-408.

Guvenen, F., 2009, “A Parsimonious Macroeconomic Model for Asset Pricing," Econometrica, 77, $1711-1750$.

Hansen, L. P., 2007, "Beliefs, Doubts and Learning: Valuing Macroeconomic Risk," American Economic Review, 97, 1-30.

Hansen, L. P., 2010, "Calibration, Empirical Evidence, and Stochastic Equilibrium Models," Slides for presentation at Cambridge INET Conference.

Harrison, J. M., and D. M. Kreps, 1978, "Speculative Investor Behavior in a Stock Market with Heterogeneous Expectations," Quarterly Journal of Economics, 92, 323-336.

Hellwig, M. F., 1980, "On the Aggregation of Information in Complete Markets," Journal of Economic Theory, 22, 477-498.

Heston, S. L., 1993, "A Closed-Form Solution for Options with Stochastic Volatility with Applications to Bond and Currency Options," Review of Financial Studies, 6, 327-343.

Isaenko, S., 2008, "The Term Structure of Interest Rates in a Pure Exchange Economy Where Investors Have Heterogeneous Recursive Preferences," The Quarterly Review of Economics and Finance, 48, 457-481.

Jouini, E., and C. Napp, 2007, "Consensus Consumer and Intertemporal Asset Pricing with Heterogeneous Beliefs," Review of Economic Studies, 74, 1149-1174.

Kogan, L., I. Makarov, and R. Uppal, 2007, "The Equity Risk Premium and the Riskfree Rate in an Economy with Borrowing Constraints," Mathematics and Financial Economics, 1, 1-19.

Kogan, L., S. A. Ross, J. Wang, and M. M. Westerfield, 2006, "The Price Impact and Survival of Irrational Traders," Journal of Finance, 61, 195-229.

Kogan, L., and R. Uppal, 2001, "Risk Aversion and Optimal Portfolio Policies in Partial and General Equilibrium Economies," Working Paper, MIT.

Longstaff, F. A., and J. Wang, 2009, "Asset Pricing and the Credit Market," Working Paper, MIT and University of California at Los Angeles.

Lucas, R. J., 1978, "Asset Prices in an Exchange Economy," Econometrica, 46, 1429-1445.

Morris, S., 1995, "The Common Prior Assumption in Economic Theory," Economics and Philosophy, 11, 227-253.

Muraviev, R., 2012, "Market Selection with Learning and Catching up with the Joneses," forthcoming in Finance and Stochastics. 
Peluso, E., and A. Trannoy, 2007, "About Sharing Rules," Working Paper, University of Verona.

Sargent, T. J., 2008, "Evolution and Intelligent Design," American Economic Review, 98, 5-37.

Scheinkman, J. A., and W. Xiong, 2003, "Overconfidence and Speculative Bubbles," Journal of Political Economy, 111, 1183-1219.

Shefrin, H., and M. Statman, 1994, "Behavioral Capital Asset Pricing Theory," Journal of Financial and Quantitative Analysis, 29, 323-349.

Stiglitz, J. E., 2010, "An Agenda for Reforming Economic Theory," Slides for presentation at Cambridge INET Conference.

Tran, N.-K., 2009, "Aggregation Biases and Asset Price Volatilities," Working Paper, MIT.

Veronesi, P., 1999, "Stock Market Overreaction to Bad News in Good Times: A Rational Expectations Equilibrium Model," Review of Financial Studies, 12, 975-1007.

Wang, J., 1993, "A Model of Intertemporal Asset Prices under Asymmetric Information," Review of Economic Studies, 60, 249-282.

Wang, J., 1996, "The Term Structure of Interest Rates in a Pure Exchange Economy with Heterogeneous Investors," Journal of Financial Economics, 41, 75-110.

Weil, P., 1989, "The Equity-Premium Puzzle and the Risk-free Rate Puzzle," Journal of Monetary Economics, 24, 401-421.

Weinbaum, D., 2009, "Investor Heterogeneity, Asset Pricing and Volatility Dynamics," Journal of Economic Dynamics and Control, 33.

Weinbaum, D., 2012, "Preference Heterogeneity and Asset Prices: An Exact Solution," Journal of Banking and Finance, 34, 2238-2246.

Xiong, W., and H. Yan, 2010, "Heterogeneous Expectations and Bond Markets," Review of Financial Studies, 23, 1433-1466.

Xiouros, C., and F. Zapatero, 2010, "The Representative Agent of an Economy with External Habit Formation and Heterogeneous Risk-Aversion," Review of Financial Studies, 23, 3017-3047.

Yan, H., 2008, "Natural Selection in Financial Markets: Does It Work?," Management Science, 54, $1935-1950$.

Zapatero, F., 1998, "Effects of Financial Innovations on Market Volatility when Beliefs are Heterogeneous," Journal of Economic Dynamics and Control, 22, 597-626.

Ziegler, A., 2007, "Why Does Implied Risk Aversion Smile," Review of Financial Studies, 20, 859-904. 